CERN-TH/2002-078

hep-ph/0204087

April 1, 2002

\title{
PHYSICS POTENTIAL AND EXPERIMENTAL CHALLENGES OF THE LHC LUMINOSITY UPGRADE
}

\begin{abstract}
Conveners: F. Gianotti ${ }^{1}$, M.L. Mangano ${ }^{2}$, T. Virdee ${ }^{1,3}$
\end{abstract}
Contributors: S. Abdullin ${ }^{4}$, G. Azuelos ${ }^{5}$, A. Ball ${ }^{1}$, D. Barberis ${ }^{6}$, A. Belyaev ${ }^{7}$, P. Bloch ${ }^{1}$, M. Bosman $^{8}$, L. Casagrande ${ }^{1}$, D. Cavalli ${ }^{9}$, P. Chumney ${ }^{10}$, S. Cittolin ${ }^{1}$, S.Dasu ${ }^{10}$, A. De Roeck ${ }^{1}$, N. Ellis $^{1}$, P. Farthouat ${ }^{1}$, D. Fournier ${ }^{11}$, J.-B. Hansen ${ }^{1}$, I. Hinchliffe ${ }^{12}$, M. Hohlfeld ${ }^{13}$, M. Huhtinen ${ }^{1}$, K. Jakobs ${ }^{13}$, C. Joram ${ }^{1}$, F. Mazzucato ${ }^{14}$, G.Mikenberg ${ }^{15}$, A. Miagkov ${ }^{16}$, M. Moretti ${ }^{17}$, S. Moretti ${ }^{2,18}$, T. Niinikoski ${ }^{1}$, A. Nikitenko ${ }^{3, \dagger}$, A. Nisati ${ }^{19}$, F. Paige ${ }^{20}$, S. Palestini ${ }^{1}$, C.G. Papadopoulos ${ }^{21}$, F. Piccinini ${ }^{2, \ddagger}$, R. Pittau ${ }^{22}$, G. Polesello ${ }^{23}$, E. Richter-Was ${ }^{24}$, P. Sharp ${ }^{1}$, S.R. Slabospitsky ${ }^{16}$, W.H. Smith ${ }^{10}$, S. Stapnes ${ }^{25}$, G. Tonelli ${ }^{26}$, E. Tsesmelis ${ }^{1}$, Z. Usubov ${ }^{27,28}$, L. Vacavant ${ }^{12}$, J. van der Bij ${ }^{29}$, A. Watson ${ }^{30}$, M. Wielers ${ }^{31}$

${ }^{1}$ Experimental Physics Division, CERN, Geneva, Switzerland

${ }^{2}$ Theoretical Physics Division, CERN, Geneva, Switzerland

${ }^{3}$ Imperial College, London, UK

${ }^{4}$ University of Maryland, USA

${ }^{5}$ Group of Particle Physics, University of Montreal, Montreal, Canada

${ }^{6}$ Dipartimento di Fisica and INFN, Università di Genova, Italy

${ }^{7}$ Florida State University, Tallahassee, FL, USA

${ }^{8}$ IFAE, Barcelona, Spain

${ }^{9}$ INFN Milano, Italy

${ }^{10}$ Univ. of Wisconsin, Madison, WI, USA.

${ }^{11}$ LAL, Orsay, France

${ }^{12}$ Lawrence Berkeley National Laboratory, Berkeley, CA, USA

${ }^{13}$ Institut für Physik, Johannes-Gutenberg Universitaet, Mainz, Germany

${ }^{14}$ Section de Physique, Université de Genève, Switzerland

${ }^{15}$ Weizman Institute, Israel

${ }^{16}$ Institute for High Energy Physics, Protvino, Russia

${ }^{17}$ Dipartimento di Fisica and INFN, Università di Ferrara, Italy

${ }^{18}$ Institute for Particle Physics Phenomenology, Durham University, UK

${ }^{19}$ Dipartimento di Fisica and INFN, Università di Roma 1, Italy

${ }^{20}$ Brookhaven National Laboratory, Upton, New York, USA.

${ }^{21}$ Institute of Nuclear Physics NCSR "Demokritos", Athens, Greece

${ }^{22}$ Dipartimento di Fisica Teorica and INFN, Università di Torino, Italy

${ }^{23}$ INFN Pavia, Italy.

${ }^{24}$ Institute of Computer Science, Jagellonian University; Institute of Nuclear Physics, Cracow, Poland

${ }^{25}$ University of Oslo, Norway

${ }^{26}$ Universitá di Pisa and INFN, Italy

${ }^{27}$ Institute of Physics, Academy of Sciences of Azerbaijan, Baku, Azerbaijan

${ }^{28}$ Joint Institute for Nuclear Research, Dubna, Russia

${ }^{29}$ Fakultät für Physik, Albert-Ludwigs Universität Freiburg, Germany

${ }^{30}$ School of Physics and Astronomy, the University of Birmingham, UK

31 TRIUMF, Vancouver, Canada

${ }^{\dagger}$ On leave of absence from ITEP, Moscow, Russia.

${ }^{\ddagger}$ On leave of absence from INFN, Sezione di Pavia, Italy. 


\begin{abstract}
We discuss the physics potential and the experimental challenges of an upgraded LHC running at an instantaneous luminosity of $10^{35} \mathrm{~cm}^{-2} \mathrm{~s}^{-1}$. The detector R\&D needed to operate ATLAS and CMS in a very high radiation environment and the expected detector performance are discussed. A few examples of the increased physics potential are given, ranging from precise measurements within the Standard Model (in particular in the Higgs sector) to the discovery reach for several New Physics processes.
\end{abstract}

\title{
Contents
}

\begin{tabular}{|lr}
\hline INTRODUCTION & 1
\end{tabular}

2 THE MACHINE UPGRADE 1

3 THE EXPECTED DETECTOR PERFORMANCE

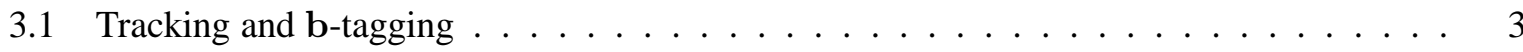

3.2 Electron identification and measurement $\ldots \ldots \ldots \ldots \ldots$. . . . . . . . . . 4

3.3 Muon identification and measurement . . . . . . . . . . . . . . . . . . 4

3.4 Forward-jet tagging and central-jet veto $\ldots \ldots \ldots \ldots \ldots \ldots$

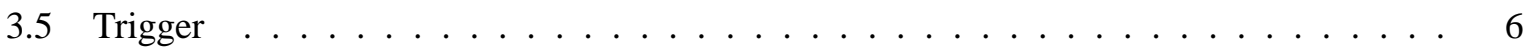

3.6 Summary of the assumptions used in this work $\ldots \ldots \ldots \ldots \ldots$

\begin{tabular}{|lll}
\hline & THE PHYSICS POTENTIAD & $\mathbf{7}$
\end{tabular}

4.1 Electroweak physics $\ldots \ldots \ldots \ldots \ldots \ldots$

$4.1 .1 \quad$ Multiple gauge boson production $\ldots \ldots \ldots \ldots \ldots$

4.1 .2 Triple gauge boson couplings $\ldots \ldots \ldots \ldots \ldots$

4.1 .3 Quartic gauge boson couplings . . . . . . . . . . . . . . . . . . . . . 9 9

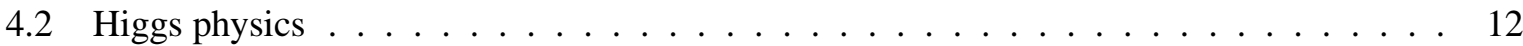

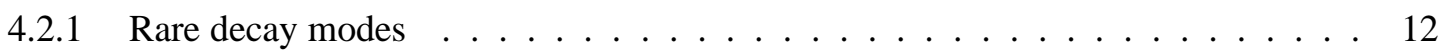

4.2 .2 Higgs couplings to fermions and bosons . . . . . . . . . . . . . . 13

$4.2 .3 \quad$ Higgs self-couplings $\ldots \ldots \ldots \ldots \ldots \ldots$

4.2 .4 The heavy Higgs bosons of the MSSM . . . . . . . . . . . . . . 17

$4.3 \quad$ Strongly-coupled vector boson system $\ldots \ldots \ldots \ldots$. . . . . . . . . . . . . . 19

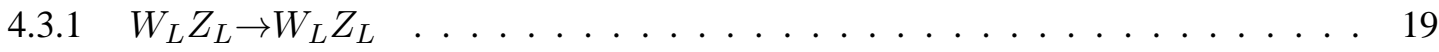

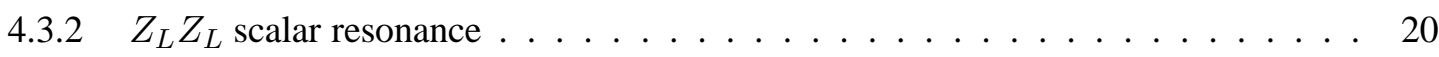

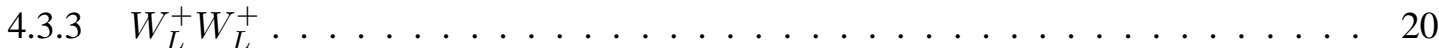

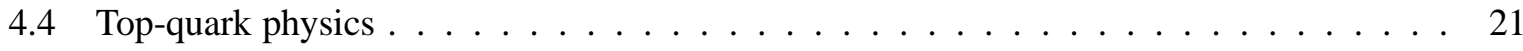

4.5 Supersymmetry . . . . . . . . . . . . . . . . . . . . . 24

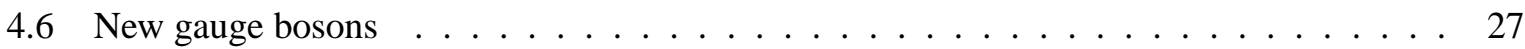

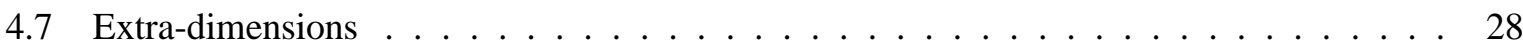

$4.7 .1 \quad$ Direct graviton production in ADD models $\ldots \ldots \ldots \ldots$. . . . . . . 28

4.7 .2 Virtual graviton exchange in ADD models . . . . . . . . . . . . . . . . . . 29 
4.7 .3 Resonance production in Randall-Sundrum models . . . . . . . . . . . . . . . . 29

4.7 .4 Resonance production in $\mathrm{TeV}^{-1}$ scale Extra-dimensions . . . . . . . . . . 30

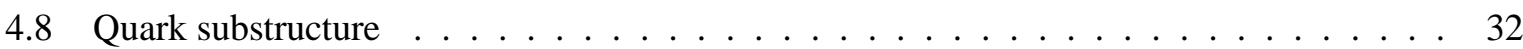

5 THE EXPERIMENTAL CHALLENGES AND THE DETECTOR R\&D 34

5.1 Inner Tracking Detector $\ldots \ldots \ldots \ldots \ldots \ldots \ldots$. . . . . . . . . . . . . . . . . . . . . . . .

$5.1 .1 \quad$ Tracking at a radius greater than $60 \mathrm{~cm} . \ldots \ldots \ldots \ldots$

5.1 .2 Tracking between a radius of $20 \mathrm{~cm}$ and $60 \mathrm{~cm} . \ldots \ldots \ldots . \ldots 37$

5.1 .3 Tracking at a radius smaller than $20 \mathrm{~cm} . \ldots \ldots \ldots \ldots \ldots$

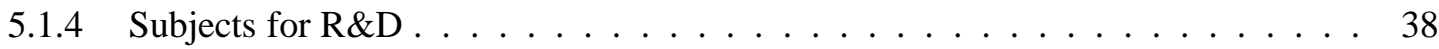

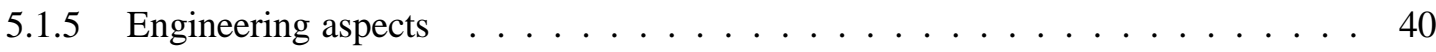

5.1 .6 Electronics . . . . . . . . . . . . . . . . 40

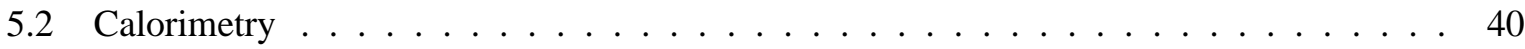

$5.2 .1 \quad$ Liquid Argon Calorimeter $\ldots \ldots \ldots \ldots \ldots \ldots$. . . . . . . . . . 41

5.2 .2 CMS Crystal ECAL . . . . . . . . . . . . . . . . . . . 43

$5.2 .3 \quad$ Plastic Scintillator Based Hadron Calorimeters ． . . . . . . . . . . . . . . . . . 44

5.2 .4 CMS Very Forward Calorimeter $\ldots \ldots \ldots \ldots$. . . . . . . . . 44

5.3 Muon Systems . . . . . . . . . . . . . . . . . . . . . 44

$5.3 .1 \quad$ Intensity considerations $\ldots \ldots \ldots \ldots \ldots \ldots \ldots$

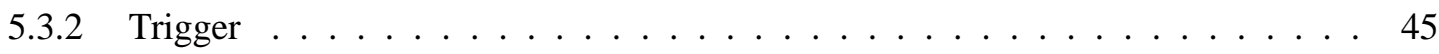

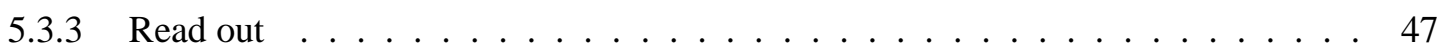

$5.3 .4 \quad$ Beam optics and radio-activation $\ldots \ldots \ldots \ldots$. . . . . . . . . . . 47

5.3 .5 Conclusion on the muon systems $\ldots \ldots \ldots \ldots$. . . . . . . . . . 47

$5.4 \quad$ Trigger and Data Acquisition $\ldots \ldots \ldots \ldots \ldots \ldots$. . . . . . . . . . . . . 48

$5.4 .1 \quad$ Higher luminosity $\ldots \ldots \ldots \ldots \ldots \ldots$. . . . . . . . . . . . . . . . . . . . . . . . . . . . .

5.4 .2 Reduced BC period $(12.5 \mathrm{~ns}) \ldots \ldots \ldots \ldots \ldots \ldots$

5.4 .3 Comments on detectors (for $12.5 \mathrm{~ns} \mathrm{BC}$ interval) $\ldots \ldots \ldots$. . . . . . . . . . 49

5.4 .4 Trigger menu $\ldots \ldots \ldots \ldots \ldots \ldots \ldots \ldots$

5.4 .5 Data Acquisition $\ldots \ldots \ldots \ldots \ldots \ldots \ldots$

5.4 .6 Main R\&D issues . . . . . . . . . . . . . . . . . . . . . . . . . 51

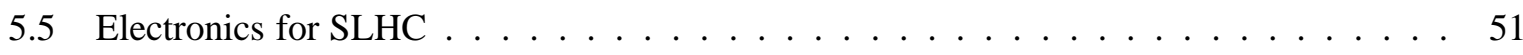

$5.5 .1 \quad$ Proposed $\mathrm{R} \& \mathrm{D}$ Projects $\ldots \ldots \ldots \ldots \ldots \ldots \ldots$

5.5 .2 Organisational Issues $\ldots \ldots \ldots \ldots \ldots$

5.6 Conclusions: Experimental Challenges and the Detector R\&D . . . . . . . . . . . . . 53

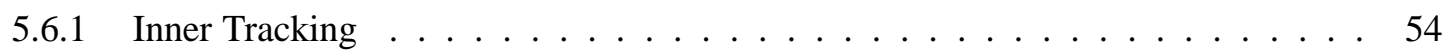

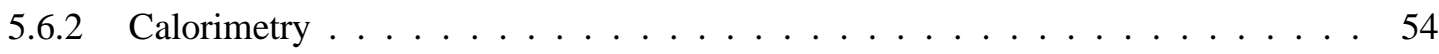

5.6 .3 Muon Systems $\ldots \ldots \ldots \ldots \ldots \ldots$

5.6 .4 Trigger and Data Acquistion $\ldots \ldots \ldots \ldots \ldots$. . . . . . . . . . . 55

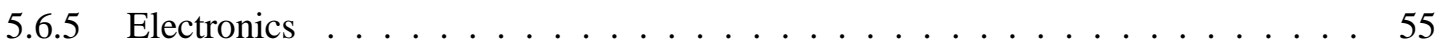

\begin{tabular}{|lll}
\hline 6 & CONCLUSIONS & 55
\end{tabular} 


\section{INTRODUCTION}

This note documents preliminary studies of the physics potential and experimental challenges of a future high-luminosity $\left(10^{35} \mathrm{~cm}^{-2} \mathrm{~s}^{-1}\right)$ upgrade of CERN's Large Hadron Collider. Hereafter, we shall refer to this upgrade as the Super-LHC (SLHC). It is impossible at this stage to give a conclusive judgement of what will be the most interesting topics to study after the first few years of LHC operation at the nominal $10^{34} \mathrm{~cm}^{-2} \mathrm{~s}^{-1}$ luminosity. We shall assume however that the physics programme of the LHC will have been accomplished, and in particular that the Higgs boson and Supersymmetry will have been found, if they are in the mass ranges expected today [1], 2]. The physics potential of the SLHC can then be roughly divided into the following main topics:

1. improvement of the accuracy in the determination of Standard Model (SM) parameters (e.g. triple (TGC) and quartic (QGC) gauge boson couplings, Higgs couplings);

2. improvement of the accuracy in the determination of parameters of New Physics possibly discovered at the LHC (e.g. sparticle spectroscopy, $\tan \beta$ measurements);

3. extension of the discovery reach in the high-mass region (e.g. quark compositeness, new heavy gauge bosons, multi-TeV squarks and gluinos, Extra-dimensions);

4. extension of the sensitivity to rare processes (e.g. FCNC top decays, Higgs-pair production, multi gauge boson production).

The detector performance at high luminosity will have a different impact on the physics output depending on the topic considered. In the case of searches at the high-mass frontier, in most cases detection of multi-TeV objects should not be impaired by the high luminosity environment. In contrast, accurate measurements of systems in the few hundred $\mathrm{GeV}$ range could be significantly affected by the large event pile-up, and reduced efficiencies or increased backgrounds could spoil the advantage of the higher luminosity. Accurate predictions will, in this case, depend on the actual detector configuration, e.g. whether a fully functional tracker can be operated at $10^{35} \mathrm{~cm}^{-2} \mathrm{~s}^{-1}$. Given the rapid progress of technology and $\mathrm{R} \& \mathrm{D}$, it is premature at this stage to attempt to select a completely defined detector scheme. Therefore, in the studies that follow, we have worked in most cases under the optimistic assumption that the main parameters of the detector performance (e.g. the $b$-tagging efficiency, the jet energy resolution) will remain the same as those expected at $10^{34} \mathrm{~cm}^{-2} \mathrm{~s}^{-1}$. To fully benefit from a tenfold increase in statistics, this is an almost mandatory requirement.

The main goal of the studies presented here is to illustrate how the SLHC could allow good progress to be made in the understanding of fundamental interactions, at a moderate extra cost relative to the overall initial LHC investment, given that the existing tunnel, accelerator and detectors would be in large part reused. We shall mostly concentrate on physics studies which are not feasible at the standard LHC. As a result, we shall not cover $B$ physics. While an increase in luminosity would in principle improve the ability to study rare $B$ decays, we expect a $B$ physics programme to be extremely unlikely at the SLHC given the difficulties to reconstruct low momentum particles.

We shall not attempt here any exhaustive cross-comparison with the potential of other machines. A discussion of different accelerator options relevant to the CERN future programme is given in [3]. A first study of the SLHC, including comparisons with the potential of an LHC energy upgrade, was presented

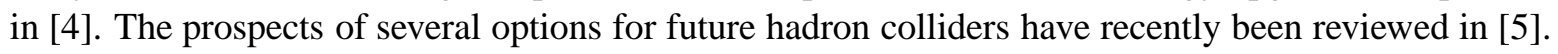

\section{THE MACHINE UPGRADE}

A feasibility study for upgrading the LHC has been launched at CERN [6], which develops scenarios for increasing both, the luminosity in each of the two high-luminosity experiments and the beam energy. The study presents some baseline options and discusses a few alternative solutions, identifying further investigations needed and proposing an $\mathrm{R} \& \mathrm{D}$ programme.

A staged upgrade of the LHC and its injectors has been considered, compatible with established accelerator design criteria and fundamental limitations of the hardware systems, aiming at a target lu- 
minosity in proton operation of $10^{35} \mathrm{~cm}^{-2} \mathrm{~s}^{-1}$ in each of the two high-luminosity experiments, and an upgrade of the centre of mass energy to $28 \mathrm{TeV}$. Three stages in the upgrading process were identified:

- Phase 0: maximum performance without hardware changes to the LHC.

- Phase 1: maximum performance while keeping the LHC arcs unchanged.

- Phase 2: maximum performance with major hardware changes to the LHC.

The nominal LHC performance for a beam energy of $7 \mathrm{TeV}$ corresponds to a total beam-beam tune spread of 0.01 , with $1.1 \times 10^{11}$ protons per bunch, yielding a luminosity of $10^{34} \mathrm{~cm}^{-2} \mathrm{~s}^{-1}$ in IP1 (ATLAS) and IP5 (CMS), halo collisions in IP2 (ALICE) and low luminosity in IP8 (LHCb). Any performance beyond these conditions will be considered as an LHC upgrade.

The steps required to reach the maximum performance without hardware changes to the accelerator (Phase 0) are:

- Collide beams only in IP1 and IP5.

- Increase the bunch population up to the beam-beam limit of $1.7 \times 10^{11}$ protons per bunch, resulting in a luminosity of $2.3 \times 10^{34} \mathrm{~cm}^{-2} \mathrm{~s}^{-1}$ at IP1 and IP5.

- Increase the main dipole field to $9 \mathrm{~T}$ (ultimate field), resulting in a maximum proton energy of $7.54 \mathrm{TeV}$. This ultimate dipole field corresponds to a beam current limited by cryogenics and by beam dump considerations.

Increasing the LHC luminosity with hardware changes only in the LHC insertions and/or in the injector complex (Phase 1) includes the following steps:

- Modify the insertion quadrupoles and/or layout to yield a $\beta^{*}$ of $0.25 \mathrm{~m}$ from the nominal $0.5 \mathrm{~m}$. In addition, although this is not the favoured option, a possible modification to the layout is to include separation dipoles closer to the interaction point to reduce the effect of long-range beambeam collisions.

- Increase the crossing angle by $\sqrt{2}$ to $424 \mu \mathrm{rad}$ from the nominal $300 \mu \mathrm{rad}$. The reason for increasing the nominal crossing angle by $\sqrt{2}$ for half the nominal $\beta^{*}$ is to keep the same small contribution of long-range collisions to the beam-beam footprint.

- Increase the bunch population up to the ultimate intensity of $1.7 \times 10^{11}$ protons per bunch, resulting in a luminosity of $3.3 \times 10^{34} \mathrm{~cm}^{-2} \mathrm{~s}^{-1}$ at IP1 and IP5.

- Upgrading the injectors to deliver beams with higher brilliance could increase the luminosity without exceeding the beam-beam limit, by increasing the product of crossing angle times bunch length. This option may yield a luminosity of up to $4 \times 10^{34} \mathrm{~cm}^{-2} \mathrm{~s}^{-1}$ with a $\beta^{*}=0.5 \mathrm{~m}$ at IP1 and IP5.

- Halving the bunch length with a new high-harmonic RF system would increase the luminosity to $4.7 \times 10^{34} \mathrm{~cm}^{-2} \mathrm{~s}^{-1}$ at IP1 and IP5.

However, there is an interesting alternative scheme to increase the LHC luminosity based on very long 'super-bunches'. This scheme would consist of the following points:

- Modify the LHC insertion quadrupoles and/or layout to reach a $\beta^{*}$ of $0.25 \mathrm{~m}$.

- Possibly increase the crossing angle to several mrad in order to pass each beam through separate final quadrupoles of reduced aperture.

- Inject a bunched beam of $1 \mathrm{~A}$ and accelerate it to $7 \mathrm{TeV}$.

- Use barrier buckets to form a single long super-bunch of 1 A current.

A 300-m long super-bunch in each of the LHC rings would be compatible with the beam-beam limit, and the corresponding luminosity in ATLAS and CMS (with alternating horizontal-vertical crossing planes) would be about $9 \times 10^{34} \mathrm{~cm}^{-2} \mathrm{~s}^{-1}$. The super-bunch option is very interesting for large crossing angles. It can potentially avoid electron cloud effects and minimize the cryogenic heat load. However, 
the associated RF manipulations and beam parameters are challenging and require further studies. This scheme requires upgrades to the detectors to achieve an effective length of between $20 \mathrm{~m}$ and $30 \mathrm{~m}$. In the following studies of physics performance and detector R\&D we shall not analyze the impact of this super-bunch option, and assume for the SLHC a $12.5 \mathrm{~ns}$ bunch crossing interval.

Finally, possible steps to increase the LHC performance with major hardware changes in the LHC arcs and/or in the injectors (Phase 2) include:

- Modify the injectors to significantly increase the brilliance to beyond its ultimate value (in conjunction with beam-beam compensation schemes).

- Equip the SPS with superconducting magnets and inject into the LHC at $1 \mathrm{TeV}$. This also implies a corresponding upgrade to the transfer lines. For given mechanical and dynamic apertures at injection, this option can increase the LHC luminosity by about a factor of two. This would also be the natural first step in view of an LHC energy upgrade, since the corresponding energy swing would be reduced by a factor of two.

- Install new superconducting dipoles in the LHC arcs to reach a beam energy of $14 \mathrm{TeV}$. Magnets with a nominal dipole field of between 16 and $16.5 \mathrm{~T}$, providing a safety margin of 1-2 T, can be considered a reasonable target for 2010 and could be operated by 2015. This requires a vigorous $\mathrm{R} \& \mathrm{D}$ programme on new superconducting materials.

More details can be found in [6]. As stated above, energy upgrades of the LHC, although being considered and being indeed most interesting from the physics point of view, require full replacement of the machine and a major R\&D activity to develop the needed dipole technology. Therefore the physics potential studies presented here were limited to the more realistic luminosity upgrade. In a few cases, however, the expected performance of a $p p$ machine running at a centre-of-mass energy of $28 \mathrm{TeV}$ is given for comparison. More results for this option can be found in Ref. [廿].

\section{THE EXPECTED DETECTOR PERFORMANCE}

The expected ATLAS and CMS performance at $10^{35} \mathrm{~cm}^{-2} \mathrm{~s}^{-1}$, and the assumptions adopted for the physics studies discussed here, are summarised below for the most relevant issues.

\subsection{Tracking and b-tagging}

It has been assumed that, provided that a large part of the inner detectors of both experiments can be replaced with more radiation hard and granular devices resulting from the R\&D described in Section 5 , reconstruction of isolated high- $p_{T}$ charged particles (e.g. muons and electrons with $p_{T}>20 \mathrm{GeV}$ ) will be possible with similar efficiency and momentum resolution as with the present detectors operating at design luminosity. Obviously, the information from the electromagnetic calorimeter and from the external Muon Spectrometer will be used to improve the performance.

The impact of the higher luminosity on b-tagging has initially been evaluated by assuming that the new pixel detectors will have the same two-track resolution as the current silicon systems. In this way, the probability of confusion in the pattern recognition remains low, and the extra (fake) b-tags are given by real tracks from the minimum bias events which are produced near the main event primary vertex and within the jet cone. The results are shown in Table 1 as a function of $p_{T}$. It can be seen that for a fixed b-tagging efficiency of $50 \%$, the rejection against light-quark jets is deteriorated by a factor of about eight at low $p_{T}\left(p_{T}<50 \mathrm{GeV}\right)$ and by a factor of less than three above $100 \mathrm{GeV}$.

Given that these results were obtained using the same response time as in the current detectors, which is a conservative assumption in view of the discussion of Section 5.1, we shall assume in most of the studies presented here that the b-tagging performance in terms of efficiency and fake rate will be the same at the SLHC as at the standard LHC. 
Table 1: Rejection against $\mathrm{u}$-jets $\left(\mathrm{R}_{u}\right)$ for a b-tagging efficiency of $50 \%$ and in various $p_{T}$ bins, as expected in ATLAS at the LHC design luminosity and with the upgraded luminosity.

\begin{tabular}{|c|c|c|}
\hline$p_{T}(\mathrm{GeV})$ & $\mathrm{R}_{u}$ at $10^{34} \mathrm{~cm}^{-2} \mathrm{~s}^{-1}$ & $\mathrm{R}_{u}$ at $10^{35} \mathrm{~cm}^{-2} \mathrm{~s}^{-1}$ \\
\hline $30-45$ & 33 & 3.7 \\
$45-60$ & 140 & 23 \\
$60-100$ & 190 & 27 \\
$100-200$ & 300 & 113 \\
$200-350$ & 90 & 42 \\
\hline
\end{tabular}

Table 2: Electron identification efficiency and rejection against jets, for $E_{T}=40 \mathrm{GeV}$, as expected in ATLAS at the LHC design luminosity and at the upgraded luminosity.

\begin{tabular}{|c|c|c|}
\hline $\mathrm{L}\left(\mathrm{cm}^{-2} \mathrm{~s}^{-1}\right)$ & Electron efficiency & Jet rejection \\
\hline $10^{34}$ & $81 \%$ & $10600 \pm 2200$ \\
$10^{35}$ & $78 \%$ & $6800 \pm 1130$ \\
\hline
\end{tabular}

\subsection{Electron identification and measurement}

An increase of a factor of ten in luminosity, and therefore in the number of pile-up minimum-bias events, increases the contribution of the pile-up noise to the calorimeter energy resolution by about a factor of three. The energy resolution of the ATLAS electromagnetic calorimeter at $10^{35} \mathrm{~cm}^{-2} \mathrm{~s}^{-1}$ has been studied with electrons of $E_{T}=30 \mathrm{GeV}$ and a full GEANT simulation which included the expected pileup. The energy resolution obtained by using also the information of the tracker is $3.6 \%$, to be compared to $2.5 \%$ at the LHC design luminosity. The deterioration is expected to be smaller at higher electron energies, because the contribution of the pile-up noise to the energy resolution decreases with the particle energy as $1 / E$.

Electron identification at $10^{35} \mathrm{~cm}^{-2} \mathrm{~s}^{-1}$ has also been studied with a full simulation of ATLAS. Table 2 compares the achieved rejection against jets faking electrons to the performance expected at the LHC design luminosity, for electrons with $E_{T}=40 \mathrm{GeV}$. For a fixed electron efficiency of about $80 \%$, the jet rejection decreases at the SLHC by about $50 \%$ compared to the standard LHC. This is mainly due to the fact that isolation cuts and shower shape criteria in the calorimeter are less powerful in the presence of a larger pile-up. As in the case of the energy resolution, the loss in jet rejection power is expected to decrease at higher electron energies.

\subsection{Muon identification and measurement}

If enough shielding can be installed in the forward regions to protect the external Muon Spectrometers from the increased radiation background, the muon reconstruction efficiency and momentum resolution provided by the muon chambers are not expected to be seriously deteriorated when running at the SLHC. The additional shielding tanslates into a reduced rapidity acceptance, which will most likely be limited to the region $|\eta|<2$. This is however not a big penalty, given the centrality of very high- $p_{T}$ final states.

\subsection{Forward-jet tagging and central-jet veto}

The presence of two jets emitted in the forward and backward regions of the detector is a distinctive signature of processes arising from $W W$ or $Z Z$ fusion, where the vector bosons are radiated by the incoming quarks. This is for instance one of the main Higgs production mechanisms at the LHC. Tagging these forward/backward jets ("forward jet tagging") is an essential handle to increase the signal-to-background ratio for these processes. Similarly, the relatively low jet activity at central rapidities in electroweak processes (e.g. Higgs production) can be used to reject QCD backgrounds (e.g. $t \bar{t}$ ). This is done by vetoing the presence of additional jets. 

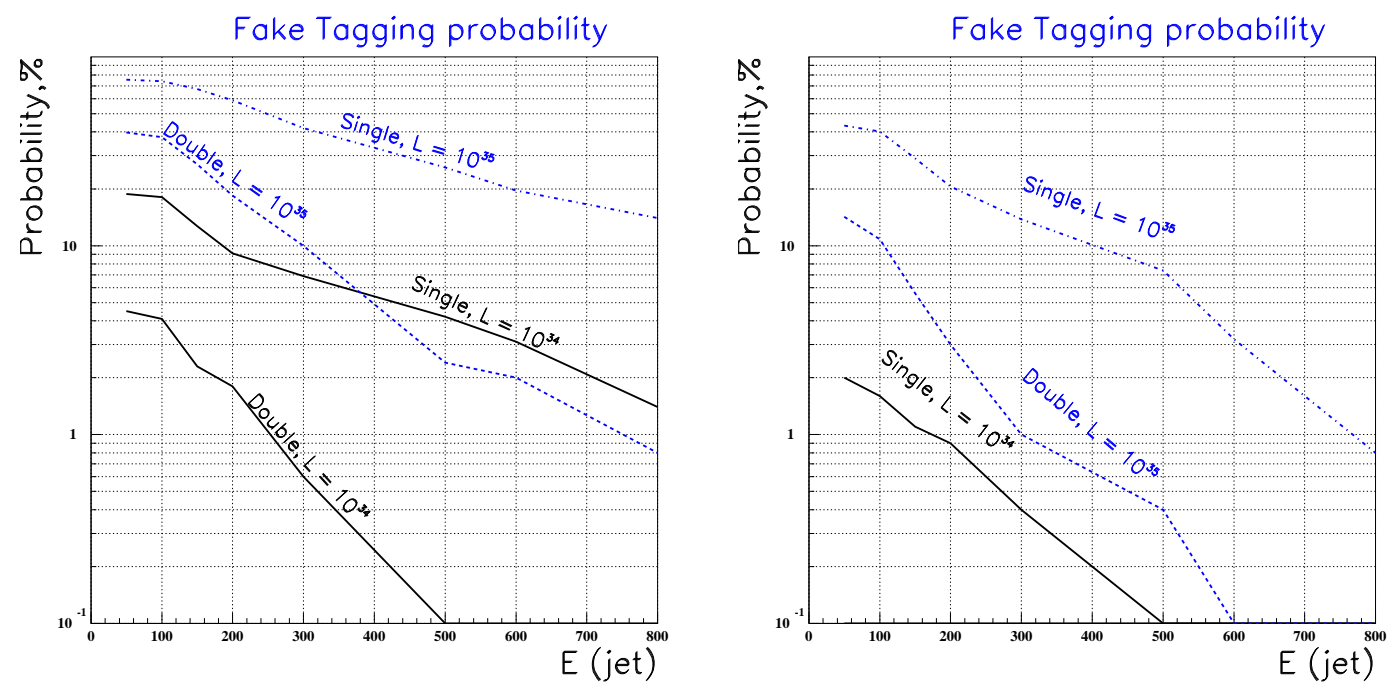

Fig. 1: Estimates of probabilities of single and double forward jet tagging from pile-up at the LHC, for the nominal and the upgraded luminosities, as a function of the jet energy, and for jet cone sizes $\Delta R=0.4$ (left) and $\Delta R=0.2$ (right).

As the luminosity increases, both these handles are expected to become less powerful, mainly because the increased pile-up can give rise to additional jets in the detector. Additional central jets overlapping purely electroweak processes spoil the efficiency of the jet veto. Similarly, additional forward jets from pile-up can mimic the typical signature of vector-boson fusion processes.

The performance of the forward jet tag and central jet veto at the SLHC was estimated in a preliminary way by using a full simulation of the ATLAS detector. The pile-up was generated by summing minimum-bias events over the correct number of bunch crossings, and by taking into account the shape of the electronic response of the various calorimeters. Jets were then found using a jet finder cone algoritm with cone sizes $\Delta R=0.4$ and $\Delta R=0.2$, and were assigned to ranges of rapidity:

- forward: $\eta>2.0$

- backward: $\eta<-2.0$

- central: $|\eta|<2$.

A single tag is defined as an event with either a forward or backward jet; a double tag has both. The probability of an event consisting only of minimum-bias interactions having either a single or double jet tag is shown in Fig. 1 as a function of the jet energy. The probability of an event having an additional central jet is shown in Fig. 2 as a function of $p_{T}$. The numbers should be compared to typical forward-jet tagging efficiencies of $\geq 80 \%$.

The main conclusion is that at a luminosity of $10^{35} \mathrm{~cm}^{-2} \mathrm{~s}^{-1}$ the forward jet tag and central jet veto performances are significantly degraded compared to design luminosity. However, if sufficiently small cone sizes are used to limit pile-up effects $(\Delta R \leq 0.2)$, these two strategies can still be effective. For instance, with a jet cone $\Delta R=0.2$ the probability of double tag is only $2 \%$ for forward jets with energy above $300 \mathrm{GeV}$. For the same cone size, an additional central jet with $p_{T}>50 \mathrm{GeV}$ is found in $10 \%$ of the cases. We stress that these results are preliminary. In particular, the fake probabilities reported in Figs. 1 and 1 depend significantly on the jet energy calibration and reconstruction conditions. Furthermore, the performance reported here may be too pessimistic as it will be possible to increase the calorimenter and trigger granularity in the forward regions, and to reduce the pile-up noise in the calorimeters by using optimal filtering techniques. Finally, possible algorithms to distinguish between incoherent pile-up of energy and QCD jets (e.g. based on the longitudinal shower profiles) have not been investigated. 

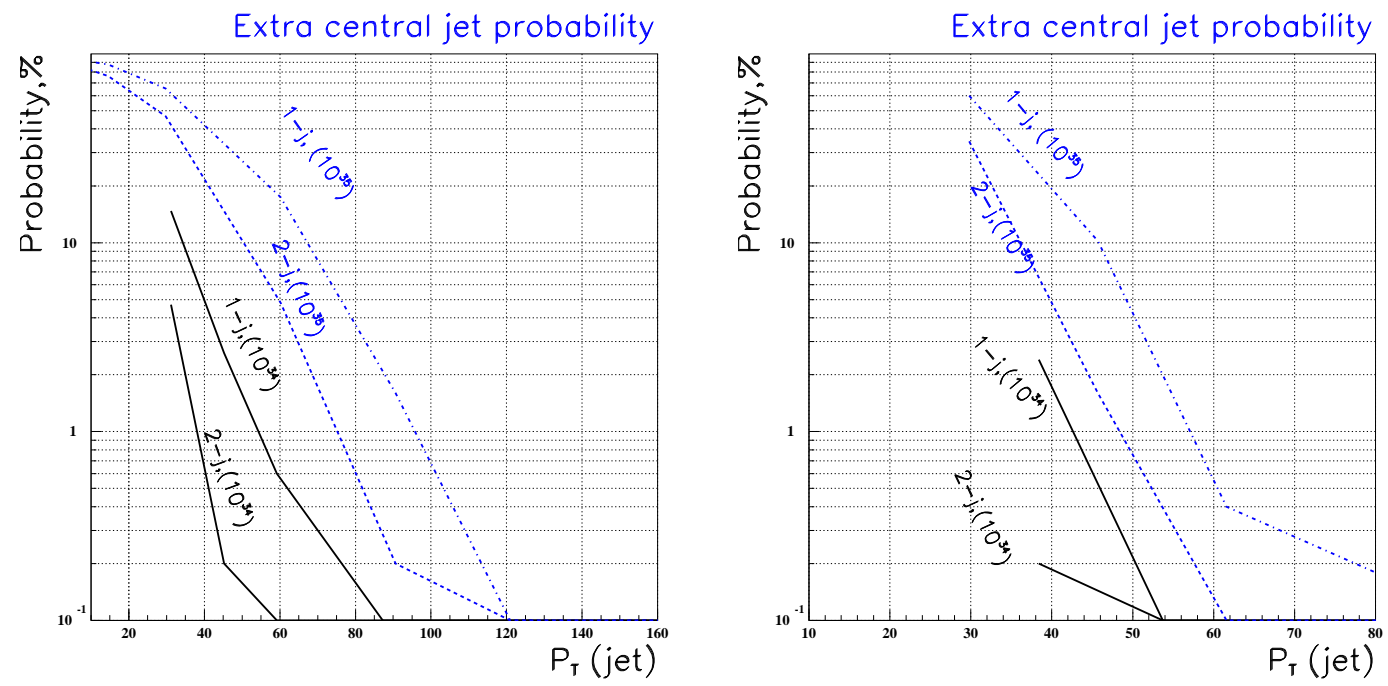

Fig. 2: Estimates of probabilities of one or two extra central jets from pile-up at the LHC, for the nominal and upgraded luminosities, as a function of the jet $p_{T}$ threshold, and for jet cone sizes $\Delta R=0.4$ (left) and $\Delta R=0.2$ (right).

\subsection{Trigger}

The strategy and expected performance of the trigger at the SLHC are discussed in Section 5.4. Here we anticipate briefly that a robust trigger menu for physics can be obtained, for an acceptable rate, by adopting the following philosophy: setting high thresholds for the inclusive triggers aiming at selecting very high $-p_{T}$ final states; pre-scaling inclusive and semi-inclusive low- $p_{T}$ triggers aiming at selecting well-known control/calibration samples (e.g. $Z \rightarrow \ell \ell$ ); using a set of exclusive menus aiming at selecting specific final states (e.g. a given decay channel of a low-mass Higgs already observed at design luminosity).

\subsection{Summary of the assumptions used in this work}

For the physics studies presented in this document, we have worked mostly under the assumption that the detector performance at $10^{35} \mathrm{~cm}^{-2} \mathrm{~s}^{-1}$ is comparable to that at $10^{34} \mathrm{~cm}^{-2} \mathrm{~s}^{-1}$. The material presented in Section 5, as well as the considerations made above, show that indeed the performance degradation is not expected to be dramatic in most cases. For example, for forward-jet tagging and central-jet veto one can recover to some extent the signal purity and background rejection by increasing the jet thresholds. We shall explicitly mention the cases in which a more conservative scenario, with degraded detector performance, has been adopted.

The detector simulations have been performed with different levels of detail. In some cases, fast simulations of fully showered final states have been employed (e.g. ATLFAST [7]). These fast simulations smear the momenta, energies and positions of the final-state particles according to the detector resolutions, as obtained from GEANT-based studies and from test beams. In other cases, the analyses are simply based on parton-level studies, with acceptances and efficiencies estimated as a function of the partons kinematics, but with no smearing for resolution effects. The precise assumptions will be listed for each individual study.

We have assumed integrated luminosities corresponding to the standard data taking time of $10^{7}$ s/year. This gives rise to total integrated luminosities for each experiment of $10^{3}\left(3 \times 10^{3}\right) \mathrm{fb}^{-1}$ for 1 (3) year(s) of running at $10^{35} \mathrm{~cm}^{-2} \mathrm{~s}^{-1}$, and $10^{2}\left(3 \times 10^{2}\right) \mathrm{fb}^{-1}$ for 1 (3) year(s) at $10^{34} \mathrm{~cm}^{-2} \mathrm{~s}^{-1}$. 
Table 3: Expected numbers of events in fully leptonic final states from multiple gauge boson production, for an integrated luminosity of $6000 \mathrm{fb}^{-1}$ and after cuts.

\begin{tabular}{|l|cccccc|}
\hline Process & $W W W$ & $W W Z$ & $Z Z W$ & $Z Z Z$ & $W W W W$ & $W W W Z$ \\
$\mathrm{~N}\left(m_{H}=120 \mathrm{GeV}\right)$ & 2600 & 1100 & 36 & 7 & 5 & 0.8 \\
\hline $\mathrm{N}\left(m_{H}=200 \mathrm{GeV}\right)$ & 7100 & 2000 & 130 & 33 & 20 & 1.6 \\
\hline
\end{tabular}

\section{THE PHYSICS POTENTIAL}

In this Section we discuss examples of physics areas where the SLHC is expected to improve on the LHC potential, either because of the extended mass reach or because of the improved measurement precision. It should be noticed that the quality and the depth of the studies presented here are by far not comparable to those of previous studies performed by ATLAS and CMS for the standard LHC and documented in Technical Proposals and Technical Design Reports. These results should therefore be considered preliminary and illustrative only.

\subsection{Electroweak physics}

The high precision studies performed at LEP have clearly indicated the essential role played by precision determinations of the electroweak (EW) parameters [8], also as a tool to look indirectly for New Physics. We show in this Section a few examples of measurements where the SLHC should improve significantly on the LHC accuracy. In addition, new channels which are rate-limited at the LHC should become accessible at the upgraded machine.

\subsubsection{Multiple gauge boson production}

Production of multiple $\left(n_{V} \geq 3\right)$ gauge bosons provides an important test of the high energy behaviour of weak interactions. The cleanest final states are those where all $W$ 's and $Z$ 's decay leptonically, but these are compromised by small branching ratios (BRs). As a rule of thumb, each additional gauge boson emitted in the hard process costs a factor of approximately 1000 in rate. As a result, luminosity limits the number of possible channels accessible at the LHC.

Table 3 shows the expectation at the SLHC. To obtain these results, we have assumed a 90\% identification efficiency for each lepton, and applied the following set of acceptance cuts:

$$
\left|\eta_{\ell}\right|<2.5, \quad p_{T}^{\ell}>20 \mathrm{GeV}
$$

All rates were evaluated at LO, using the parton distribution set CTEQ5M and renormalization and factorization scales $\mu_{R}=\mu_{F}=\sum_{i=1, n_{W}+n_{Z}} m_{V_{i}}$. NLO predictions are available in the case of diboson production [11], leading to $K$ factors of the order of $1.3,1.5$ and 1.8 for $Z Z, W^{+} W^{-}$and $W^{ \pm} Z$, respectively [9]. Similarly, $K$ factors of the order of 1.5 are expected for the higher multiplicities.

From Table 3 it can be seen that, although with limited statistics, the channels $W^{ \pm} Z Z \rightarrow 5 \ell, Z Z Z \rightarrow 6 \ell$ and even the four-gauge-boson final state $W^{+} W^{-} W^{+} W^{-}$become accessible at the SLHC. As a result, the first limits on 5-ple gauge boson vertices (expected to vanish in the SM) could be set. The standard LHC luminosity would not allow these channels to be oberved. All rates are increased in presence of a Higgs boson above threshold for the decay into boson pairs (see the row corresponding to $m_{H}=200 \mathrm{GeV}$ in Table 3).

In addition to the direct production of multiple gauge bosons, the SLHC luminosity should allow also an accurate measurement of triple boson production in boson-boson fusion (allowing, again, a test of the quintuple couplings). For example, for the $W Z$ fusion processes

$$
Z W^{ \pm} \rightarrow W^{+} W^{-} W^{ \pm}
$$

870 fully leptonic events are expected for $m_{H}=120 \mathrm{GeV}$ and $6000 \mathrm{fb}^{-1}$. In the case of a $200 \mathrm{GeV} \mathrm{Higgs,}$ including the resonant production of $W$ pairs leads to 2700 events. 


\subsubsection{Triple gauge boson couplings}

As discussed in [9], the LHC will significantly improve the precision of the measurements of the Triple Gauge boson Couplings (TGC) compared to the LEP and Tevatron results. In the SM, the TGC's are uniquely fixed by gauge invariance and renormalizability. Extensions to the SM, in which for example the gauge bosons are not elementary but are bound states of more fundamental particles, generically lead to deviations from the SM prediction for the TGCs. The larger the available statistics, the higher the sensitivity to these deviations. In the case of a positive indication of non-SM TGC's at the LHC, increased statistics at the SLHC should allow a deeper understanding of which specific realization of New Physics is responsible for these deviations. The latter are in fact parameterized by effective interactions which, in order to preserve unitarity at high energy, require the inclusion of form factors. The mass scale $\Lambda$ which is needed to define such form factors is typically associated to the scale at which New Physics manifests itself. A measurement of the energy dependence of the TGC's will probe the shape of the form factor [9], and therefore allow extraction of the value of the scale $\Lambda$. As a result, there is no limit a priori to the accuracy one would like to achieve in the determination of TGCs: the larger the precision, the stronger the reach in searches for phenomena beyond the Standard Model.

Assuming electromagnetic gauge invariance and $\mathrm{C}$ - and P-conservation, five parameters can be used to describe the triple-gauge vertices: $g_{1}^{Z}, \Delta \kappa_{\gamma}, \Delta \kappa_{Z}, \lambda_{\gamma}$, and $\lambda_{Z}$ [9]. The SM values of these parameters are one for $g_{1}^{Z}$ and zero for the others, at the tree level.

At the LHC two processes can be studied to access these TGCs: $W \gamma \rightarrow \ell \nu \gamma$ production, which probes the couplings $\lambda_{\gamma}$ and $\Delta \kappa_{\gamma}$, and $W Z \rightarrow \ell \nu \ell \ell$ production, which probes the couplings $\lambda_{Z}, \Delta \kappa_{Z}$ and $g_{1}^{Z}$. After simple kinematic cuts and lepton and photon identification, a few thousands events are expected for an integrated luminosity of $100 \mathrm{fb}^{-1}$ in one experiment, with a background contamination of 20-30\%. In contrast, the $W W \rightarrow \ell \nu \ell \nu$ process, which also proceeds through triple-gauge interactions in the $s$-channel, suffers from a large $t \bar{t}$ background and therefore has not been considered here.

The experimental sensitivity to anomalous TGC's arises from the increase of the production crosssection and from alteration of differential distributions. The $\lambda$-type couplings have a strong $\sqrt{s}$ dependence, being enhanced at high centre-of-mass energy. Therefore they can be constrained by measuring the total cross-section for the above-mentioned processes and by looking for an excess of gauge boson pairs with high $p_{T}$ compared to the SM expectation. The $\kappa$-type couplings, on the other hand, modify mainly the angular distributions of the final state particles.

For a luminosity of $10^{35} \mathrm{~cm}^{-2} \mathrm{~s}^{-1}$, the analysis reported here uses conservatively only final states containing muons and photons, because these final states do not necessitate significant detector upgrades. This choice entails a loss of 50\% (75\%) of the $W \gamma(W Z)$ effective rate. In addition, only transverse momentum distributions have been used to constrain TGC's, therefore these results are pessimistic in the case of the $\kappa$-type couplings.

The expected sensitivity is summarised in Table 4 and Fig. 3 for different luminosity scenarios. For comparison, the performance of a $p p$ machine running at $\sqrt{s}=28 \mathrm{TeV}$ is also shown. Only statistical errors have been taken into account. The dominant systematic uncertainty is expected to come from higher-order QCD corrections. Their contribution, which has not been evaluated for the studies presented here, depends on the extent to which they can be constrained by using theory and data and controlled by applying a jet veto at $10^{35} \mathrm{~cm}^{-2} \mathrm{~s}^{-1}$.

It can be seen that a tenfold luminosity increase should extend the sensitivity for the $\lambda$-type and $g_{1}^{Z}$ parameters into the range $(\sim 0.001)$ expected from radiative corrections in the Standard Model, and should therefore allow a meaningful test of these corrections and others that arise for example in Supersymmetric models. It should also be noted that, even in the pessimistic approach adopted here and with only one experiment, the precision on the $\lambda$-type and $g_{1}^{Z}$ parameters is equal to or better than that expected at a Linear Collider with $\sqrt{s}=500 \mathrm{GeV}$ and an integrated luminosity of $500 \mathrm{fb}^{-1}$ [10]. On the other hand even the SLHC is not competitive with a Linear Collider for the measurement of the $\kappa$-type 
Table 4: Expected 95\% C.L. constraints on Triple Gauge Couplings in ATLAS for various luminosity/energy scenarios $(\Lambda=10 \mathrm{TeV}$ ). Only one coupling is allowed to vary at the time, while the others are fixed at their SM values. The last column shows the expectation for a Linear Collider with $\sqrt{s}=500 \mathrm{GeV}$ and $500 \mathrm{fb}^{-1}$ [10].

\begin{tabular}{|c|c|c|c|c|c|}
\hline Coupling & $\begin{array}{c}14 \mathrm{TeV} \\
100 \mathrm{fb}^{-1}\end{array}$ & $\begin{array}{c}14 \mathrm{TeV} \\
1000 \mathrm{fb}^{-1}\end{array}$ & $\begin{array}{c}28 \mathrm{TeV} \\
100 \mathrm{fb}^{-1}\end{array}$ & $\begin{array}{c}28 \mathrm{TeV} \\
1000 \mathrm{fb}^{-1}\end{array}$ & $\begin{array}{c}\mathrm{LC} \\
500 \mathrm{fb}^{-1}, 500 \mathrm{GeV}\end{array}$ \\
\hline$\lambda_{\gamma}$ & 0.0014 & 0.0006 & 0.0008 & 0.0002 & 0.0014 \\
\hline$\lambda_{Z}$ & 0.0028 & 0.0018 & 0.0023 & 0.009 & 0.0013 \\
\hline$\Delta \kappa_{\gamma}$ & 0.034 & 0.020 & 0.027 & 0.013 & 0.0010 \\
\hline$\Delta \kappa_{Z}$ & 0.040 & 0.034 & 0.036 & 0.013 & 0.0016 \\
\hline$g_{1}^{Z}$ & 0.0038 & 0.0024 & 0.0023 & 0.0007 & 0.0050 \\
\hline
\end{tabular}

parameters, which do not exhibit a strong energy dependence and which are optimally constrained by angular measurements in the clean environment of an $e^{+} e^{-}$machine.

\subsubsection{Quartic gauge boson couplings}

Quartic boson couplings (QGC) are an essential component of the EW theory. Similarly to the TGCs, they are required by gauge invariance and their values are uniquely determined within the SM by the value of the EW gauge coupling. As in the case of TGC's, possible deviations from the SM prediction are parametrised in terms of effective terms in the Lagrangian.

The results presented here are based on the work of Ref. [12], where the following operators leading to genuine quartic vertices are considered:

$$
\begin{aligned}
\mathcal{L}_{4} & =\alpha_{4}\left[\operatorname{Tr}\left(V_{\mu} V_{\nu}\right)\right]^{2} \\
\mathcal{L}_{5} & =\alpha_{5}\left[\operatorname{Tr}\left(V_{\mu} V^{\mu}\right)\right]^{2} \\
\mathcal{L}_{6} & =\alpha_{6} \operatorname{Tr}\left(V_{\mu} V_{\nu}\right) \operatorname{Tr}\left(T V^{\mu}\right) \operatorname{Tr}\left(T V^{\nu}\right), \\
\mathcal{L}_{7} & =\alpha_{7} \operatorname{Tr}\left(V_{\mu} V^{\mu}\right)\left[\operatorname{Tr}\left(T V^{\nu}\right)\right]^{2} \\
\mathcal{L}_{10} & =\frac{\alpha_{10}}{2}\left[\operatorname{Tr}\left(T V_{\mu}\right) \operatorname{Tr}\left(T V_{\nu}\right)\right]^{2} .
\end{aligned}
$$

In the unitary gauge, there are new anomalous contributions to the $Z Z Z Z$ vertex coming from all five operators, to the $W^{+} W^{-} Z Z$ vertex from all operators except $\mathcal{L}_{10}$, and to the $W^{+} W^{-} W^{+} W^{-}$vertex from $\mathcal{L}_{4}$ and $\mathcal{L}_{5}$. A possible way to probe these couplings is via the scattering of gauge bosons in reactions like $p p \rightarrow q q V V \rightarrow V V j j$ [13, 14, 15], with $V=W^{ \pm}$or $Z$.

Table 5 shows the limits on the couplings $\alpha_{i}(i=4,5,6,7,10)$ expected at the LHC, as a function of integrated luminosity, compared to current indirect limits from Ref. [16]. Fully leptonic final states were required and the cuts applied are those of Eq. (11). It can be seen that in few cases the improvement obtained with the luminosity upgrade goes beyond the simple statistical scaling. This is due to the fact that almost no events are expected in the $Z Z$ final state at $10^{34} \mathrm{~cm}^{-2} \mathrm{~s}^{-1}$. The interplay among the various channels, and the correlations among different parameters $\alpha_{i}$, are shown in Figs. 46 .

In addition to the vector boson scattering processes, an alternative probe of quartic couplings is given by the production of three gauge bosons via the off-resonance production of a $\mathrm{W}$ or $\mathrm{Z}$ decaying into a system of three gauge bosons $\left(V^{*} \rightarrow V V V\right)$. In this case, a different kinematical configuration is probed. For vector boson scattering, two of the bosons are space-like, with virtualities of the order of $M_{W}$; for triple gauge boson production, one is off-shell but is time-like and with large virtuality. The observation of anomalies in the two channels would therefore provide complementary information, and would also be sensitive to different combinations of the QGC parameters. For triple gauge boson production we updated the studies presented in [9], assuming a total integrated luminosity of $6000 \mathrm{fb}^{-1}$. Given the number of events quoted in Table 3, and using just the $Z Z Z$ final state, the limit $\left|\alpha_{4}+\alpha_{5}\right|<0.025$ was obtained at 95\% C.L. for $\Lambda=2 \mathrm{TeV}$. This is to be compared with 0.09 with $100 \mathrm{fb}^{-1}$. 

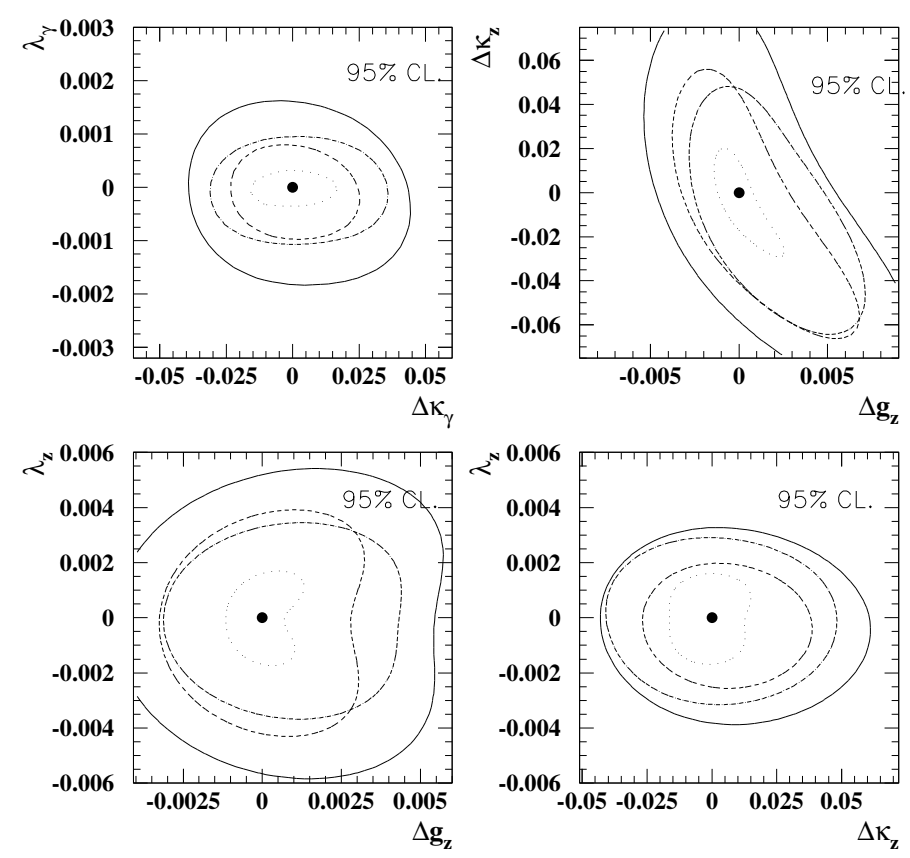

Fig. 3: Expected 95\% C.L. constraints on Triple Gauge Couplings in ATLAS, resulting from two-parameter fits $(\Lambda=10$ TeV). The contours correspond to $14 \mathrm{TeV}$ and $100 \mathrm{fb}^{-1}$ (solid), $28 \mathrm{TeV}$ and $100 \mathrm{fb}^{-1}$ (dot dash), $14 \mathrm{TeV}$ and $1000 \mathrm{fb}^{-1}$ (dash) and $28 \mathrm{TeV}$ and $1000 \mathrm{fb}^{-1}$ (dotted).

Table 5: $1 \sigma$ limits on the anomalous quartic couplings $\alpha_{i}$ at LHC and SLHC (95\% C.L. limits are also given in this case), as well as the present indirect bounds from Ref. [16].

\begin{tabular}{|c|c|c|c|c|}
\hline Coupling & $\begin{array}{c}\text { Indirect Limits } \\
(1 \sigma) \\
\left(\times 10^{-3}\right)\end{array}$ & $\begin{array}{c}\text { LHC, } 100 \mathrm{fb}^{-1} \\
(1 \sigma) \\
\left(\times 10^{-3}\right)\end{array}$ & $\begin{array}{c}\text { LHC, } 6000 \mathrm{fb}^{-1} \\
(1 \sigma) \\
\left(\times 10^{-3}\right)\end{array}$ & $\begin{array}{c}\text { LHC, } 6000 \mathrm{fb}^{-1} \\
95 \% \text { C.L. } \\
\left(\times 10^{-3}\right)\end{array}$ \\
\hline$\alpha_{4}$ & $-120 . \leq \alpha_{4} \leq 11$. & $-1.1 \leq \alpha_{4} \leq 11$. & $-0.67 \leq \alpha_{4} \leq 0.74$ & $-0.92 \leq \alpha_{4} \leq 1.1$ \\
\hline$\alpha_{5}$ & $-300 . \leq \alpha_{5} \leq 28$. & $-2.2 \leq \alpha_{5} \leq 7.7$ & $-1.2 \leq \alpha_{5} \leq 1.2$ & $-1.7 \leq \alpha_{5} \leq 1.7$ \\
\hline$\alpha_{6}$ & $-20 . \leq \alpha_{6} \leq 1.8$ & $-9.6 \leq \alpha_{6} \leq 9.1$ & $-3.5 \leq \alpha_{6} \leq 3.2$ & $-4.3 \leq \alpha_{6} \leq 3.9$ \\
\hline$\alpha_{7}$ & $-19 . \leq \alpha_{7} \leq 1.8$ & $-10 . \leq \alpha_{7} \leq 7.4$ & $-4.4 \leq \alpha_{7} \leq 2.2$ & $-5.4 \leq \alpha_{7} \leq 2.8$ \\
\hline$\alpha_{10}$ & $-21 . \leq \alpha_{10} \leq 1.9$ & $-24 . \leq \alpha_{10} \leq 24$. & $-4.1 \leq \alpha_{10} \leq 4.1$ & $-4.8 \leq \alpha_{10} \leq 4.8$ \\
\hline
\end{tabular}



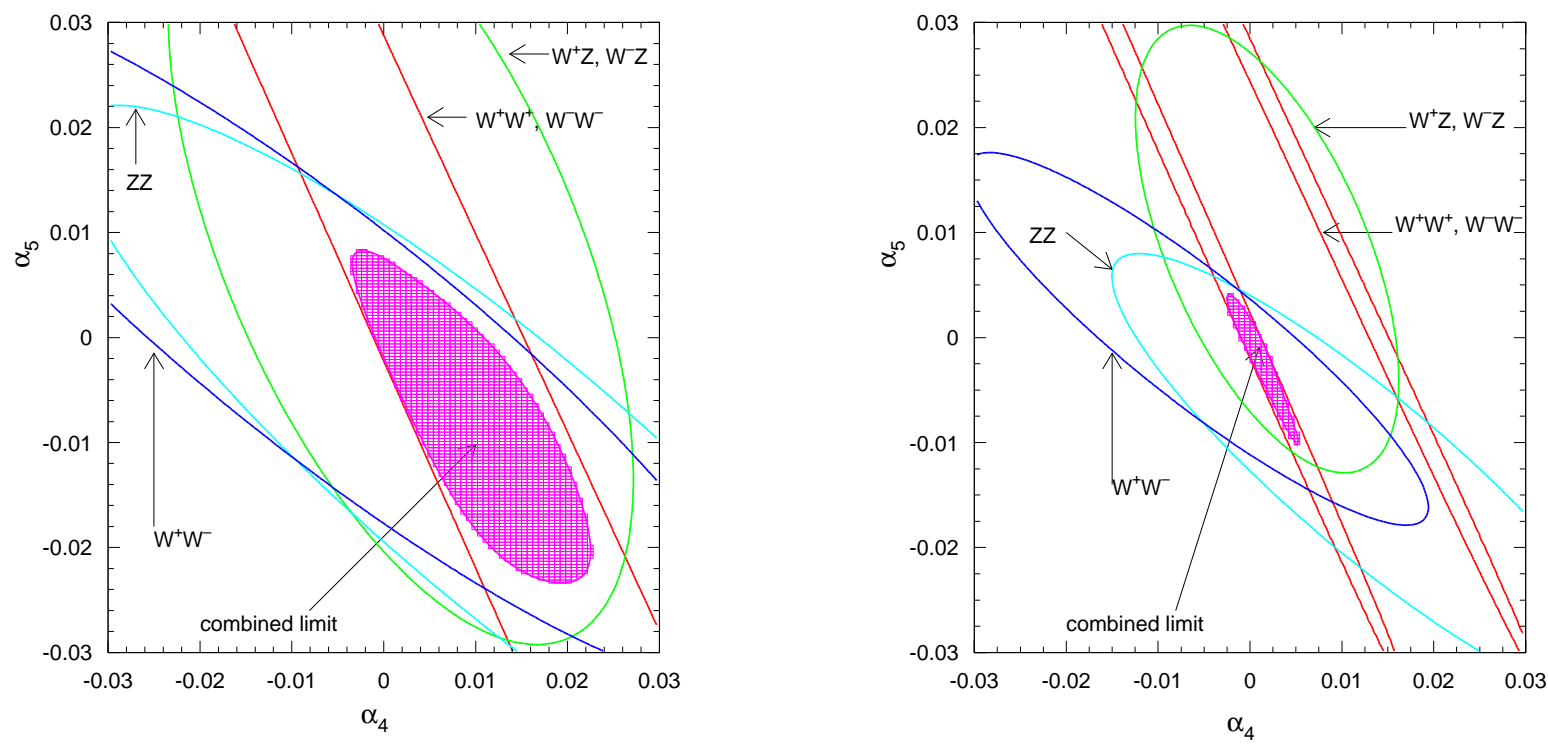

Fig. 4: 1- $\sigma$ contours in the $\alpha_{4}-\alpha_{5}$ plane for $W^{+} W^{-}, W^{ \pm} W^{ \pm}, W^{ \pm} Z$ and $Z Z$ production for an integrated luminosity of $100 \mathrm{fb}^{-1}$ (left plot) and $6000 \mathrm{fb}^{-1}$ (right plot). We have assumed $\alpha_{6}=\alpha_{7}=\alpha_{10}=0$.
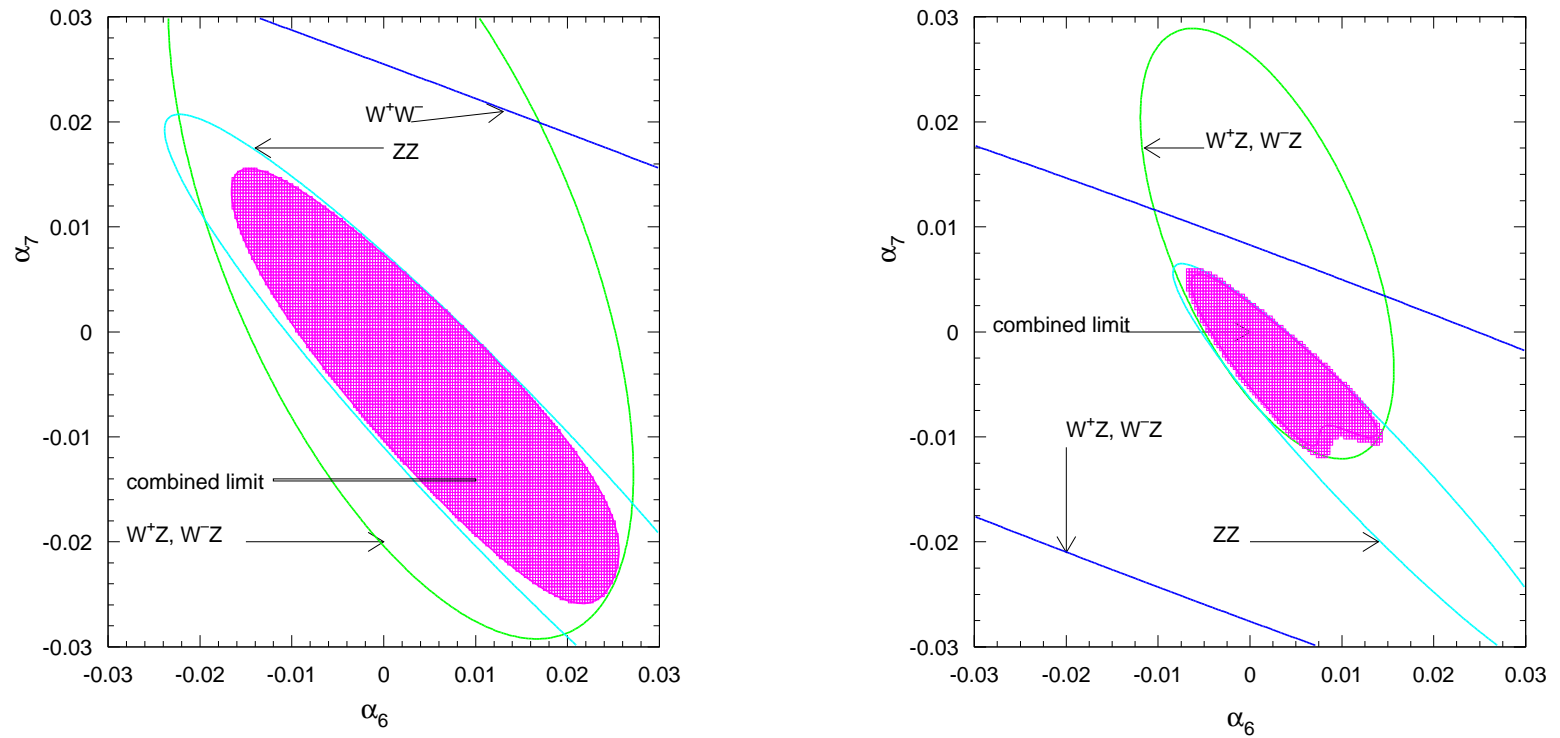

Fig. 5: $1-\sigma$ contours in the $\alpha_{6}-\alpha_{7}$ plane from $W^{+} W^{-}, W^{ \pm} Z$ and and $Z Z$ production for an integrated luminosity of $100 \mathrm{fb}^{-1}$ (left plot) and $6000 \mathrm{fb}^{-1}$ (right plot). We have assumed $\alpha_{4}=\alpha_{5}=\alpha_{10}=0$. 

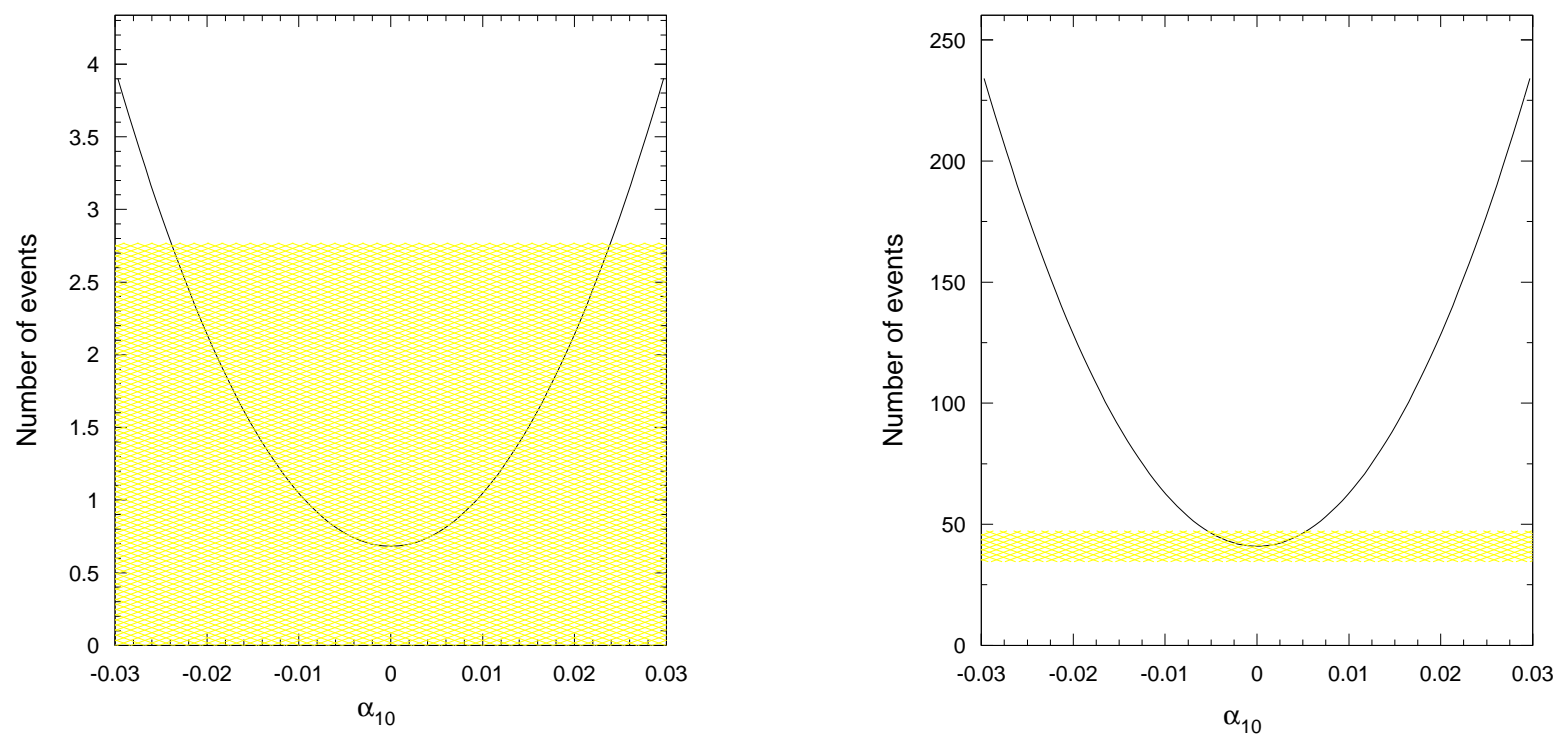

Fig. 6: Expected number of events from $Z Z$ production as a function of $\alpha_{10}$ for an integrated luminosity of $100 \mathrm{fb}^{-1}$ (left plot) and $6000 \mathrm{fb}^{-1}$ (right plot). The horizontal line represents a 64\% C.L. effect. We have assumed $\alpha_{4}=\alpha_{5}=\alpha_{6}=\alpha_{7}=0$.

\subsection{Higgs physics}

The Higgs search programme, including the discovery of a SM Higgs over the full allowed mass range or of at least one SUSY Higgs boson, will be largely accomplished at the standard LHC. There are issues where the LHC potential is limited by statistics and where a luminosity upgrade could therefore have a significant impact.

Four examples are discussed in this Section. The possibility of observing a Higgs boson in decay channels which are rate-limited at the LHC; the measurements of Higgs couplings to fermions and bosons with improved precision; the possibility of observing the production of Higgs pairs and measuring Higgs self-couplings; the increased discovery potential for heavy MSSM Higgs bosons in the difficult region of the decoupling limit. It should be stressed that Higgs physics requires fully functional detectors, providing powerful $b$-tagging and electron identification and allowing precise measurements of particles with moderate energies. Major detector upgrades are therefore needed in this case for the SLHC phase.

\subsubsection{Rare decay modes}

Two examples of channels which are accessible with difficulty at the LHC because of their tiny rates are discussed here. Assuming that a Higgs boson will have been previously discovered in higher-rate final states, these rare decay modes can be used to extend the information available on the Higgs couplings to fermions and bosons.

The possible decay $H \rightarrow Z \gamma$ of a SM Higgs is relevant only in the mass region $100-160 \mathrm{GeV}$ and has a branching ratio of a few per mill. In contrast to the $H \rightarrow \gamma \gamma$ channel, which has a similar branching ratio, an additional suppression in the $Z \gamma$ case comes from the fact that only decays of the $Z$ into electron or muon pairs lead to observable final states above the background at the LHC. Taking this into account, the production cross-section times branching ratio for $H \rightarrow Z \gamma \rightarrow \ell \ell \gamma$ is only $\sim 2.5 \mathrm{fb}$, too small to be observed at the LHC. Indeed, the expected significance for $600 \mathrm{fb}^{-1}$ (300 $\mathrm{fb}^{-1}$ per experiment) is $\sim 3.5 \sigma$. A factor of ten in luminosity, i.e. $6000 \mathrm{fb}^{-1}$, would allow the observation of a signal at the $\sim 11 \sigma$ level. This additional channel would provide an additional measurement of the Higgs couplings to bosons (see Section 4.2.2).

Another interesting decay mode which may become accessible at the SLHC is $H \rightarrow \mu^{+} \mu^{-}$. 
Table 6: Expected signal significance of a SM $g g \rightarrow H \rightarrow \mu \mu$ signal for various mass values, as obtained by combining ATLAS and CMS and for an integrated luminosity of $3000 \mathrm{fb}^{-1}$ per experiment [19]. The expected statistical accuracy on the measurement of the product of cross-section times BR is also given.

\begin{tabular}{|l|c|c|}
\hline$m_{H}(\mathrm{GeV})$ & $S / \sqrt{B}$ & $\frac{\delta \sigma \times \mathrm{BR}(H \rightarrow \mu \mu)}{\sigma \times \mathrm{BR}}$ \\
\hline $120 \mathrm{GeV}$ & 7.9 & 0.13 \\
$130 \mathrm{GeV}$ & 7.1 & 0.14 \\
$140 \mathrm{GeV}$ & 5.1 & 0.20 \\
$150 \mathrm{GeV}$ & 2.8 & 0.36 \\
\hline
\end{tabular}

This channel, which in the SM has a BR of order $10^{-4}$, has recently been studied for the LHC design luminosity in [18] using production via gauge boson fusion, and in [19] using production via $g g$ fusion. The analyses were performed at the generator level, taking into account the experimental acceptance and muon momentum resolution. The large background from $Z \rightarrow \mu \mu$ was reduced by constructing a signal likelihood function based on the $\mu \mu$ system kinematics and the event $E_{T}$ distribution. It should also be noticed that, since a Higgs signal will have been previously observed in higher-rate decay modes, the Higgs mass will be known with an accuracy of $\sim 0.1 \%$. Therefore the $\mathrm{Z}$ background can be precisely measured in the signal region by using a sample of $Z \rightarrow e e$ decays. Due to the small branching ratio, this channel cannot be observed to better than $3.5 \sigma$ at the LHC design luminosity, even combining both production channels. Extrapolation to the SLHC gives, for the $g g$-fusion channel alone, the results of Table 6. In the mass range $120-140 \mathrm{GeV}$, a $5 \sigma$ evidence or larger can be obtained, and the square root of the production cross-section times branching ratio (which is directly proportional to the muon Yukawa coupling $g_{H \mu \mu}$ ) could be measured with statistical accuracies of better than $10 \%$. These results are comparable to those obtained for a $200 \mathrm{TeV}$ Very Large Hadron Collider (VLHC) with an integrated luminosity of $300 \mathrm{fb}^{-1}$. The possibility of adding the contribution of the gauge-boson fusion production channel rests on the viability of the forward jet tagging, and has so far not been explored in detail for the SLHC.

\subsubsection{Higgs couplings to fermions and bosons}

Assuming that a SM Higgs boson will have been discovered at the LHC, measurements of Higgs couplings to fermions and bosons should be possible [17], but in most cases the precision will be limited by the available statistics [20]. A luminosity upgrade should therefore be useful for this physics.

In principle, the Higgs coupling for instance to a given fermion family $f$ could be obtained from the following relation:

$$
R(H \rightarrow f f)=\int L d t \cdot \sigma(p p \rightarrow H) \cdot \frac{\Gamma_{f}}{\Gamma}
$$

where $R(H \rightarrow f f)$ is the Higgs production rate in a given final state, which can be measured experimentally, $\int L d t$ is the integrated luminosity, $\sigma(p p \rightarrow H)$ is the Higgs production cross-section, and $\Gamma$ and $\Gamma_{f}$ are the total and partial Higgs widths respectively. Hence, a measurement of the Higgs production rate in a given channel allows extraction of the partial width for that channel, and therefore of the Higgs coupling $g_{f}$ to the involved decay particles $\left(\Gamma_{f} \sim g_{f}^{2}\right.$ ), provided that the Higgs production cross-section and the total Higgs width are known from theory.

Model-independent measurements are only possible if one considers ratios of couplings, which are experimentally accessible through the measurements of ratios of rates for two different final states, because in the ratio the total Higgs cross-section, width and luminosity cancel. Examples are shown in Fig. 7. The left plot gives the expected precision on the ratio of the Higgs widths for the decays into $W W$ and $Z Z$. For masses larger than approximately $150 \mathrm{GeV}$ a comparison of the $H \rightarrow Z Z \rightarrow 4 \ell$ and $H \rightarrow W W \rightarrow \ell \nu \ell \nu$ rates provides a direct measurement of $\Gamma_{W} / \Gamma_{Z}$. At smaller masses the process 

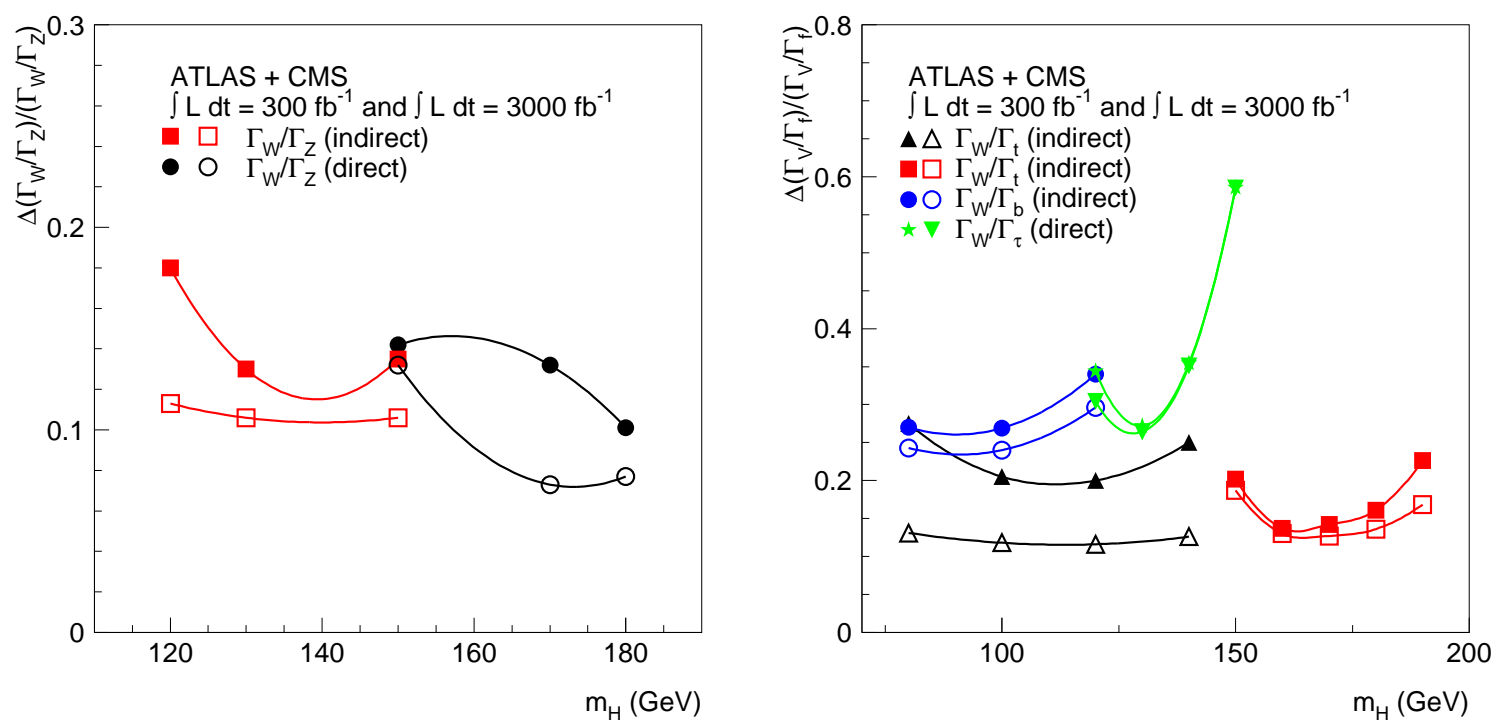

Fig. 7: Expected uncertainties on the measured ratios of the Higgs widths to final states involving bosons only (left) and bosons and fermions (right), as a function of the Higgs mass. Closed symbols: two experiments and $300 \mathrm{fb}^{-1}$ per experiment (standard LHC); open symbols: two experiments and $3000 \mathrm{fb}^{-1}$ per experiment (SLHC). Direct and indirect measurements have been included (see text).

$H \rightarrow W W \rightarrow \ell \nu \ell \nu$ has too low a rate but one can use the measured rate of $H \rightarrow \gamma \gamma$ to extract $\Gamma_{W}$, at the prize of introducing some theoretical assumptions (indirect measurement). The coupling $H \rightarrow \gamma \gamma$ is dominated by a loop graph with an intermediate $W$ and hence the rate of $H \rightarrow \gamma \gamma$ can be related to the $H W W$ coupling. Similarly, the right plot in Fig. 7 shows the expected precisions on the measurements of ratios of Higgs couplings to fermions and bosons. The coupling $H t \bar{t}$ can be probed in the mass region below $150 \mathrm{GeV}$ by comparing the $W H \rightarrow \ell \nu \gamma \gamma$ rate and the $H \rightarrow \gamma \gamma$ rate. The latter production rate is determined by the coupling of the Higgs to gluon pairs (since $g g \rightarrow H$ is the production mechanism) and the dominant contribution to this coupling is from a top quark loop. An indirect measurement of the ratio of couplings $H W W$ and $H t \bar{t}$ can therefore be performed. In the mass region above $150 \mathrm{GeV}, \Gamma_{W} / \Gamma_{t}$ can be obtained in a similar way by using the $W H \rightarrow W W W$ and the $H \rightarrow W W$ channels. Since in this mass range the dominant systematics is the theoretical uncertainty on the absolute cross-sections for the two independent production channels, the higher luminosity leads to a minor improvement. Progress in the understanding of the theoretical systematics will however allow to take full benefit of the higher statistics. The processes $t \bar{t} H(\rightarrow \gamma \gamma)$ and $t \bar{t} H(\rightarrow b \bar{b})$ can be combined to give the ratio of widths to $W W$ and to $b \bar{b}$ in an indirect way. Finally, measurements of the $H \rightarrow \tau \tau$ and $H \rightarrow W W \rightarrow \ell \nu \ell \nu$ rates in events with tagged forward jets, which arise from the fusion process $q q \rightarrow q q H$, can be combined to directly obtain $\Gamma_{W} / \Gamma_{\tau}$. At this time, the impact of the $10^{35} \mathrm{~cm}^{-2} \mathrm{~s}^{-1}$ environment on the combined request of tau identification, missing $E_{T}$ and forward jets has not been evaluated, and we do not have an estimate of the improvement possible at the SLHC for this channel.

It can be seen that at the SLHC ratios of Higgs couplings to fermions and bosons should be measured with precisions of $10 \%$ or better in most cases. In some cases, this represents an improvement by up to a factor of two on the ultimate precision expected at the standard LHC. Progress for other channels can be anticipated as a result of improved theoretical understanding of the Higgs production mechanisms, and of the impact of the experimental environment on the detector performance.

\subsubsection{Higgs self-couplings}

A complete determination of the parameters of the Standard Model requires the measurements of the Higgs selfcouplings. These include a trilinear and a quartic interaction. In the SM, the corresponding 


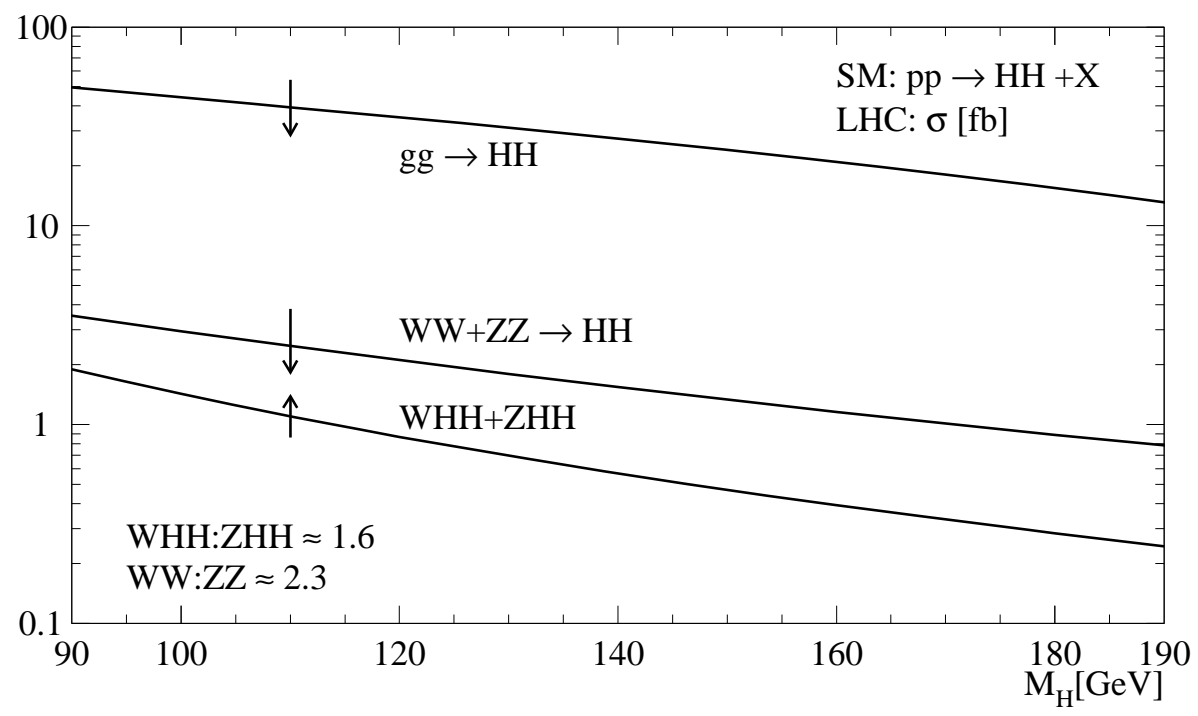

Fig. 8: Cross-sections for the production of Higgs boson pairs from $g g$ fusion, $W W / Z Z$ fusion and double Higgs-strahlung (from [21]). The vertical arrows correspond to a variation of $\lambda_{H H}$ from 1/2 (arrow's tail) to 3/2 (arrow's tip) of the SM value. couplings are fixed at LO in terms of the Higgs mass and vacuum expectation value $v$ :

$$
\lambda_{H H H}^{S M}=3 \frac{m_{H}^{2}}{v}, \quad \lambda_{H H H H}^{S M}=3 \frac{m_{H}^{2}}{v^{2}}
$$

A direct measurement of $\lambda_{H H H}$ can be obtained via the detection of Higgs pair production, where a contribution is expected from the production of a single off-shell Higgs which decays into a pair of Higgs. This contribution will always be accompanied by diagrams where the two Higgs bosons are radiated independently, with couplings proportional to the Yukawa couplings or to the gauge couplings. As a result, different production mechanisms will lead to different sensitivities of the $H H$ rate to the value of $\lambda_{H H H}$. The production mechanisms which have been considered in the literature in the context of hadron-hadron collisions include [21]:

1. inclusive $H H$ production, dominated by the partonic process $g g \rightarrow H H$ [22, 23]

2. vector boson fusion [24]: $q q \rightarrow q q V^{*} V^{*}$, followed by $V^{*} V^{*} \rightarrow H H$ (where possible different quark flavours are understood in both initial and final state)

3. associated production with $W$ or $Z$ bosons [25]: $q \bar{q} \rightarrow V H H$

4. associated production with top quark pairs: $g g / q \bar{q} \rightarrow t \bar{t} H H$

In theories beyond the SM, alternative production channels may exist. For example, when several Higgs multiplets exist, as is typical of Supersymmetry, pairs of lighter Higgs bosons can be produced in the resonant decay of a heavier one. In this document we concentrate on the SM case.

The $H H$ production rates are shown in Fig. 8 for the first three channels [21], and in Table 7 for the $t \bar{t} H H$ case $\rrbracket$. The arrows indicate the variation in rate expected when changing the self-coupling in the range $\lambda_{H H H}^{S M} / 2<\lambda_{H H H}<3 / 2 \lambda_{H H H}^{S M}$. Depending on the value of $m_{H}$, different decay channels dominate. For $m_{H} \lesssim 140 \mathrm{GeV} H \rightarrow b \bar{b}$ decays dominate, for $170 \lesssim m_{H} \lesssim 190 \mathrm{GeV} H \rightarrow W W$ dominates, and for larger masses $W W$ and $Z Z$ final states give the largest rate.

Given the extremely low production rates, and the potentially large backgrounds associated to the final states with the largest signal rates, naive arguments lead to the conclusion that detection of SM Higgs pairs at $10^{34} \mathrm{~cm}^{-2} \mathrm{~s}^{-1}$ is not feasible. As a result, no complete study of the backgrounds is present in the literature. We present here the first preliminary results of studies performed specifically for the $10^{35} \mathrm{~cm}^{-2} \mathrm{~s}^{-1}$ option. We analysed the cases of production via gluon-gluon fusion, vector boson fusion,

\footnotetext{
${ }^{1}$ C.G. Papadopoulos, unpublished, using results of [26].
} 
Table 7: Total production cross-section (fb) fb for $p p \rightarrow t \bar{t} H H$, as a function of $m_{H}$ and of $\lambda_{H H H}$ (given in units of the SM value $\left.\lambda_{H H H}^{S M}\right)$.

\begin{tabular}{|l|cccc|}
\hline$m_{H}(\mathrm{GeV})$ & $\sigma\left(\lambda_{H H H}=1\right)$ & $\sigma\left(\lambda_{H H H}=1 / 2\right)$ & $\sigma\left(\lambda_{H H H}=2\right)$ & $\sigma\left(\lambda_{H H H}=0\right)$ \\
\hline 120 & 1.0 & 0.9 & 1.3 & 0.8 \\
140 & 0.54 & 0.48 & 0.73 & 0.42 \\
160 & 0.32 & 0.32 & 0.47 & 0.24 \\
\hline
\end{tabular}
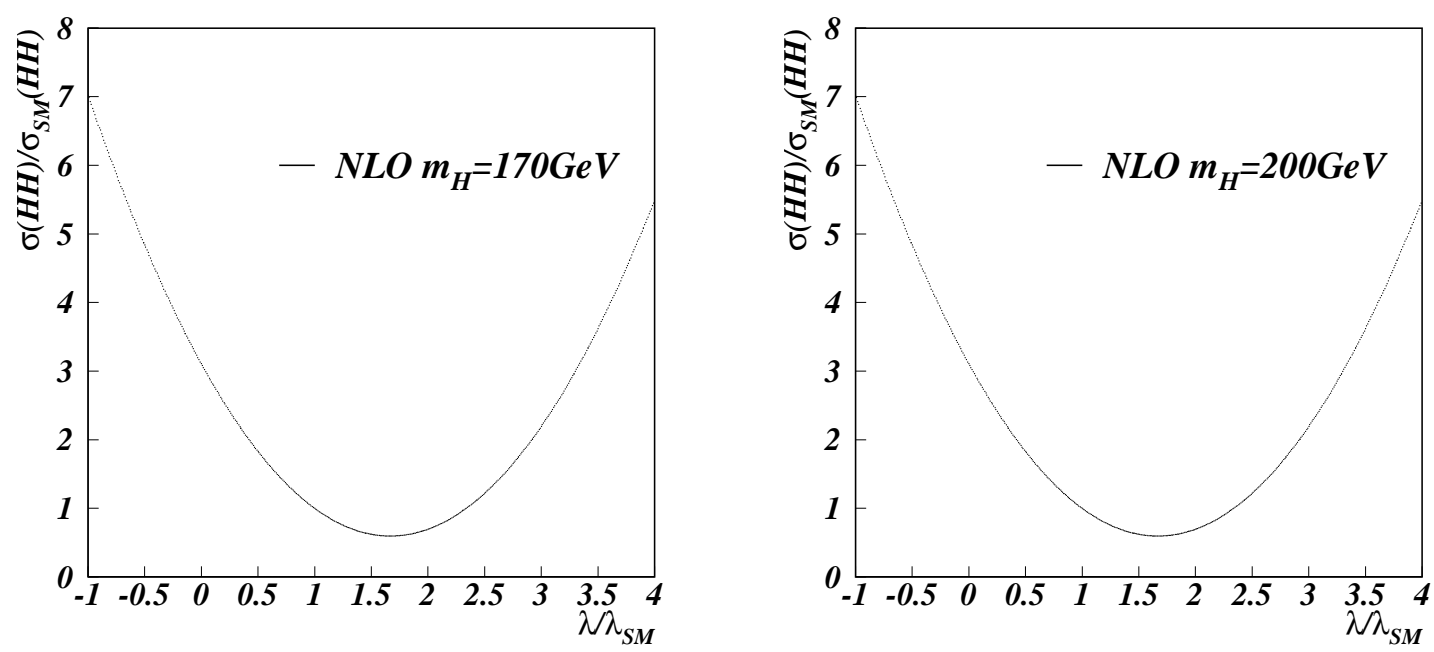

Fig. 9: $\lambda_{H H_{H}}$ dependence of the $g g \rightarrow H H$ rate [23], for $m_{H}=170 \mathrm{GeV}$ (left) and $200 \mathrm{GeV}$ (right).

and associated production with top quark pairs. In the latter two cases, we concluded that the large level of backgrounds make it very hard, if not impossible, to extract meaningful signals. These findings will be documented in more detail elsewhere. In the case of the gluon fusion channel, we shall now show that the extra factor of ten in luminosity, if accompanied by a detector performance comparable to that expected at the LHC, allows extraction of a signal,and provides the first measurements of $\lambda_{H H H}$, if the Higgs lies within the mass range $170 \mathrm{GeV}<m_{H}<200 \mathrm{GeV}$.

\section{$\mathrm{gg} \rightarrow \mathbf{H H}$}

The $g g$ fusion process has a strong dependence on the value of $\lambda_{H H H}$. This is shown in Fig. 9] [23]. For small $m_{H}$, $4 b$ final states dominate. In spite of the double resonance, the QCD bacgkround is immense and limits any chance to observe a signal. Among all channels we considered, the most interesting one turned out to be

$$
g g \rightarrow H H \rightarrow W^{+} W^{-} W^{+} W^{-} \rightarrow \ell^{ \pm} \nu j j \ell^{ \pm} \nu j j
$$

which has a good overall $\mathrm{BR}$ for $m_{H} \gtrsim 170 \mathrm{GeV}$. The like-sign lepton requirement is essential to reduce the high-rate opposite-sign lepton final states from Drell Yan (DY) and $t \bar{t}$. Potential backgrounds to this signal arise from:

1. $t \bar{t}+$ jets, where the second lepton comes from $b$ decays

2. $W Z+$ jets, where one of the leptons from the $Z$ is not identified

3. $t \bar{t} W$

4. $W W W j j$, including the resonant channel $W(H \rightarrow W W) j j$

5. $t \bar{t} t \bar{t}$

All these backgrounds are in principle reducible, since they share one or more of the following features not present in the signal: presence of $b$-jets; presence of additional hard and isolated leptons; jet-jet invariant masses not consistent with $W$ decays. 
Table 8: Expected numbers of signal and background events after all cuts for the $g g \rightarrow H H \rightarrow 4 W \rightarrow \ell^{+} \ell^{\prime+} 4 j$ final state, for $\int \mathcal{L}=6000 \mathrm{fb}^{-1}$.

\begin{tabular}{|l|ccccccc|}
\hline$m_{H}$ & Signal & $t \bar{t}$ & $W^{ \pm} Z$ & $W^{ \pm} W^{+} W^{-}$ & $t \bar{t} W^{ \pm}$ & $t \bar{t} t \bar{t}$ & $S / \sqrt{B}$ \\
\hline $170 \mathrm{GeV}$ & 350 & 90 & 60 & 2400 & 1600 & 30 & 5.4 \\
$200 \mathrm{GeV}$ & 220 & 90 & 60 & 1500 & 1600 & 30 & 3.8 \\
\hline
\end{tabular}

To reduce the top-quark backgrounds, all events with one (or more) $b$-tags are rejected (this is accomplished by reweigthing each event with a factor of 0.5 for each $b$ jet with $E_{T}>30 \mathrm{GeV}$ and $|\eta|<2.4$ ). To select hadronic $W$ decays we require the presence of at least two jet pairs with invariant masses $50<m_{j j}<110 \mathrm{GeV}$. To reduce the contribution of leptons from untagged $b$ decays we apply an isolation cut. We assumed a 10\% identification inefficiency for either leptons, to estimate the residual DY contamination from non-reconstructed DY pairs. In addition, the following cuts are applied:

$$
p_{T}^{\ell}>20 \mathrm{GeV}, \quad\left|\eta_{\ell}\right|<2.4 \quad \forall \ell
$$

$$
\geq 4 \text { jets with } E_{T}>20 \mathrm{GeV} \text { and }|\eta|<2.4 \text {, two of which with } E_{T}>30 \mathrm{GeV}
$$

To reduce the high jet multiplicity $4 t$ processes, we finally ask that there be at most six jets with $E_{T}>30 \mathrm{GeV}$ in the event. Backgrounds 1-3 were generated with PYTHIA, and processed through ATLFAST; backgrounds 3-5 were evaluated using parton-level simulations based on exact multi-particle matrix elements, following [27]. We used the channel $t \bar{t} W$, for which simulations were carried out using both approaches, to cross-check our overall results. We also verified that a PYTHIA+ATLFAST simulation of the $q \bar{q}^{\prime} \rightarrow W H$ contribution to the $W W W j j$ background is consistent with a partonic simulation of the $q \bar{q}^{\prime} \rightarrow W H$ subset of the full set of contributing processes.

The resulting event numbers after all cuts are shown in Table 8 . In spite of the signal being smaller than the backgrounds, the number of events is large enough to provide a statistical excess of 5.3 (3.8) $\sigma$ for $m_{H}=170$ (200) GeV. It is important to remark that the precise size of the backgrounds is subject today to large theoretical uncertainties, and the above significance values should be taken as indicative only. However, once the data will be available these uncertainties can be determined experimentally by using control samples or by other tools. For example, the largest backgrounds $\left(t \bar{t} W^{ \pm}\right.$and $\left.W^{ \pm} W^{+} W^{-} j j\right)$ have a potentially measurable charge asymmetry. The ratios $\sigma\left(X W^{+}\right)-\sigma\left(X W^{-}\right) / \sigma\left(X W^{+}\right)+\sigma\left(X W^{-}\right)$ (with $X=t \bar{t}, W^{+} W^{-} j j$ ) are quite insensitive to theoretical uncertainties (scales, parton densities, etc), thereby allowing a determination of the background normalization. Additional handles for an accurate estimate of the backgrounds come from counting events where some of the cuts are relaxed (e.g. the $b$-veto, the lepton misidentification). We therefore assume that the uncertainty on the background subtraction will be dominated by statistics. In the case of SM Higgs production, this leads to a determination of the total production cross-section with a statistical uncertainty of $\pm 26 \%( \pm 20 \%)$ for $m_{H}=200 \mathrm{GeV}$ $\left(m_{H}=170 \mathrm{GeV}\right)$. This allows a measurement of $\lambda_{H H H}$ with statistical errors of $25 \%$ (19\%).

\subsubsection{The heavy Higgs bosons of the MSSM}

The LHC discovery potential for MSSM Higgs bosons decaying into SM particles is summarised in Fig. 10 in the usual $\tan \beta$ vs $m_{A}$ plane. This plot shows that over a good part of the parameter space (note the logaritmic scale) the LHC should be able to discover two or more SUSY Higgs bosons, except in the region at large $m_{A}$ (the so-called "decoupling limit"). In this region, only the lightest Higgs boson $h$ can be observed, unless the heavier Higgs bosons $\left(H, A, H^{ \pm}\right)$have detectable decay modes into SUSY particles. This means that the LHC cannot promise a complete and model-independent observation of the heavy part of the MSSM Higgs spectrum, although the observation of sparticles (e.g. squarks and gluinos) will clearly indicate that additional Higgs bosons should exist.

In the region of the decoupling limit, the heavy Higgs bosons are not accessible at a future Linear Collider like TESLA either. In this case, however, very precise measurements of the $h$ parameters 


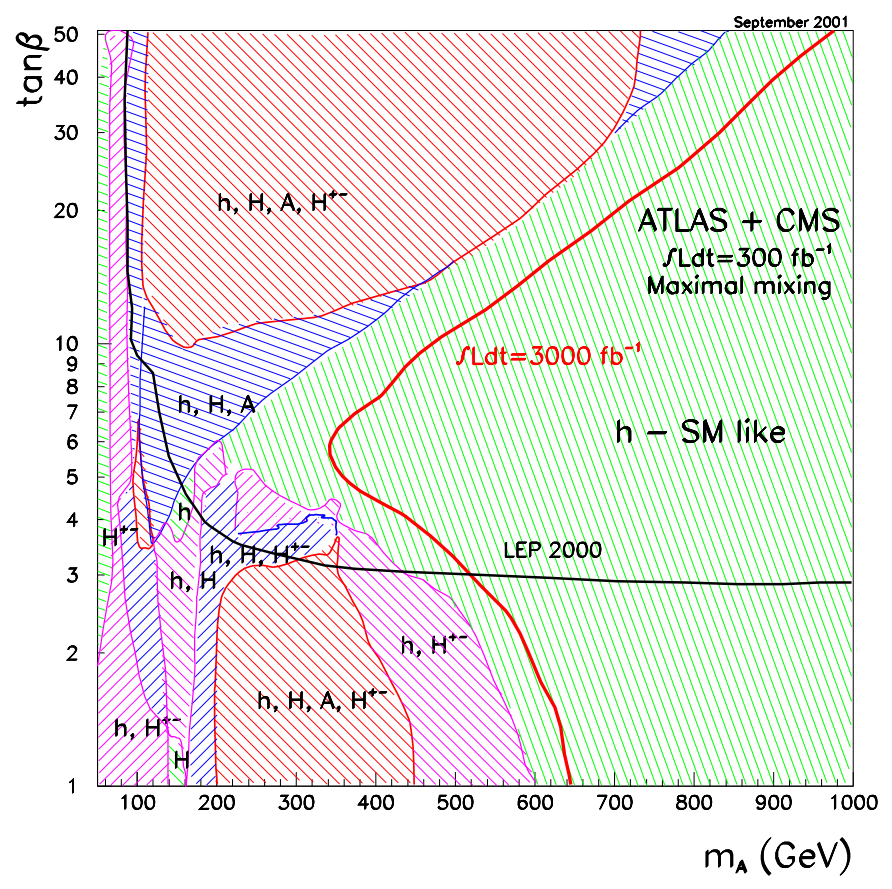

Fig. 10: Regions of the MSSM parameter space where the various Higgs bosons can be discovered at $\geq 5 \sigma$ at the LHC (for $300 \mathrm{fb}^{-1}$ per experiment and both experiments combined) through their decays into SM particles. In the dashed regions at least two Higgs bosons can be discovered, whereas in the dotted region only $h$ can be discovered at the LHC. In the region to the left of the rightmost contour at least two Higgs bosons can be discovered at the SLHC (for $3000 \mathrm{fb}^{-1}$ per experiment and both experiments combined). 
Table 9: Cross-sections for backgrounds to the $W_{L} Z_{L} \rightarrow W_{L} Z_{L}$ process.

\begin{tabular}{|c|c|}
\hline Process & $\sigma(\mathrm{pb})$ \\
\hline$q q \rightarrow q q W Z$ & 1.45 \\
$Z b \bar{b}$ & 141 \\
$Z t \bar{t}$ & 2.23 \\
$q q \rightarrow W Z$ & 3.00 \\
$q q \rightarrow Z Z$ & 0.81 \\
\hline
\end{tabular}

should be able to demonstrate indirectly (i.e. through radiative corrections), and limited to the region $m_{A}<500 \mathrm{GeV}$, the existence of heavier Higgs bosons [10]. Figure 10 also shows that the SLHC should be able to extend significantly the region over which at least one heavy Higgs boson can be discovered at $\geq 5 \sigma$ in addition to $h$ (rightmost contour in the plot), covering in particular almost the full part of the parameter space where TESLA should be able to constrain (at the 95\% C.L.) the heavy part of the SUSY Higgs spectrum through precise measurements.

\subsection{Strongly-coupled vector boson system}

If there is no light Higgs boson, then general arguments [28] imply that scattering of electroweak gauge bosons at high energy will show structure beyond that expected in the Standard Model. In order to explore such signals it is necessary to measure final states containing pairs of gauge bosons with large invariant mass.

\subsection{1 $W_{L} Z_{L} \rightarrow W_{L} Z_{L}$}

Estimates of the production of a $\rho$-like vector resonance of $W_{L} Z_{L}$ can be obtained from the Chiral Lagrangian model, with the inverse amplitude method of unitarization [9, 29]. The cross-section depends, in next to leading order, on a linear combination $a_{4}-2 a_{5}$ of two quadrilinear coupling parameters. The model was implemented in PYTHIA. Only the channel $W_{L} Z_{L} \rightarrow W_{L} Z_{L} \rightarrow \ell \nu \ell^{+} \ell^{-}$is considered here, although the resonance can be produced in the $q \bar{q}$ fusion channel at higher rate. Forward jet tagging is here an essential ingredient to reduce the background.

The irreducible Standard Model background $q q \rightarrow q q W Z$, with transverse gauge bosons in the final state, was generated with COMPHEP [30] with cuts $p_{T}(q, W, Z)>15 \mathrm{GeV}$, and $m_{W Z}>500 \mathrm{GeV}$, with CTEQ5L structure functions and $Q=m_{Z}$. The process includes electroweak and QCD diagrams, as well as the quadrilinear gauge boson couplings. The Higgs mass was set at the low value of $100 \mathrm{GeV}$, and the signal is then defined, as in [31], as the enhancement of the SM prediction over the $100 \mathrm{GeV}$ Higgs. Other backgrounds considered were $Z b \bar{b}$ and $Z t \bar{t}$, also generated with COMPHEP, with cuts $p_{T}(b, t)>15 \mathrm{GeV}$ and $p_{T}(Z)>50 \mathrm{GeV}$, and SM production of $W Z, Z Z$, generated with PYTHIA. Table 9 gives the cross-sections for the different backgrounds.

The selection criteria are based on leptonic cuts:

$$
\begin{aligned}
p_{T}\left(\ell_{1}\right)>150 \mathrm{GeV}, \quad p_{T}\left(\ell_{2}\right)>100 \mathrm{GeV}, \quad p_{T}\left(\ell_{3}\right) & >50 \mathrm{GeV} \\
\left|m\left(\ell_{1} \ell_{2}\right)-m_{Z}\right| & <10 \mathrm{GeV} \\
E_{T}^{m i s s} & >75 \mathrm{GeV}
\end{aligned}
$$

and forward jet tagging, i.e. the presence of one forward and one backward jet $(|\eta|>2)$ with energy greater than 300 (400) $\mathrm{GeV}$ at LHC (SLHC) luminosity. In addition, events with jets with transverse momenta greater than 50 (70) $\mathrm{GeV}$ at LHC (SLHC) luminosity in the central region $(|\eta|<2$ ) were rejected. The degraded jet tag and jet veto performances discussed in Section 3.4 were used for the SLHC case. 

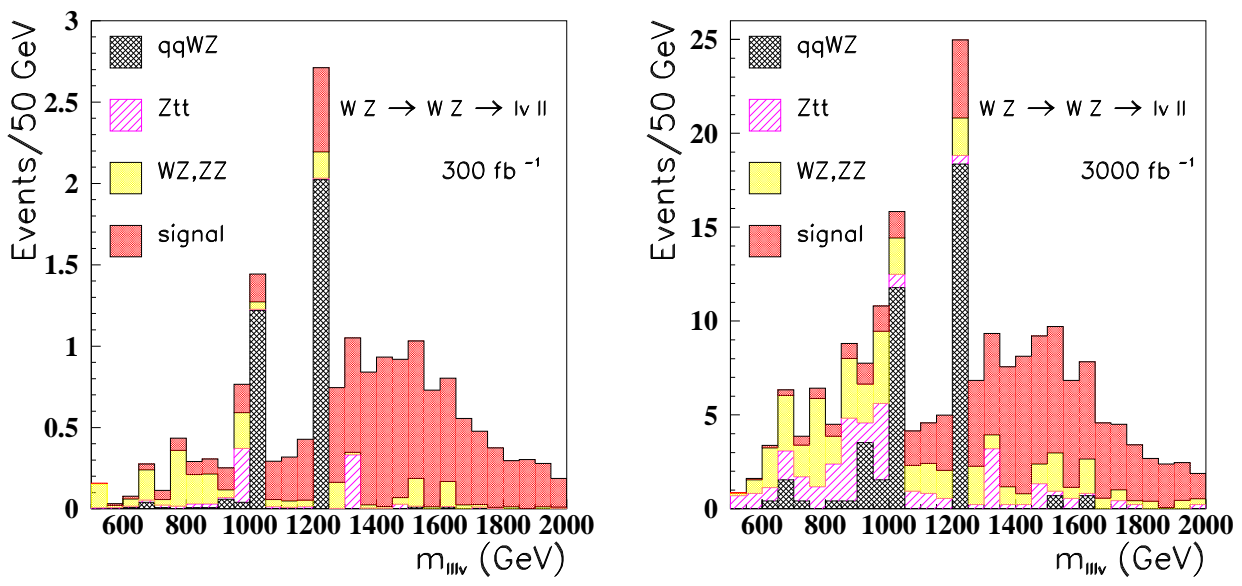

Fig. 11: Expected signal and background for a $1.5 \mathrm{TeV}$ WZ resonance in the leptonic decay channel for $300 \mathrm{fb}^{-1}$ (left) and $3000 \mathrm{fb}^{-1}$ (right).

Figure 11 shows the expected signal for a $1.5 \mathrm{TeV}$ resonance, corresponding to the choice of the Chiral Lagrangian parameters of eqs. (3)-(円) $\alpha_{4}-2 \alpha_{5}=0.006$, at the LHC and at the SLHC. The resonance is at the limit of the observation at LHC, with 6.6 events expected over a background of about 2.2 events around the region of the peak, but at the SLHC the signal has a significance of $S / \sqrt{B} \sim 10$.

\subsection{2 $Z_{L} Z_{L}$ scalar resonance}

Production of a scalar $Z_{L} Z_{L}$ resonance decaying via the gold-plated channel $Z Z \rightarrow 4 \ell$ is a rare process, well suited to the SLHC. As in the case of the $W Z$ resonance discussed above, the Chiral Lagrangian model with regularization by the inverse amplitude method was used. The cross-section depends on a linear combination, $7 \alpha_{4}+11 \alpha_{5}$, of the same parameters as those of the vector resonance, and therefore observation of the scalar resonance will resolve $\alpha_{4}$ and $\alpha_{5}$ unambiguously.

Production occurs through the scattering processes $W_{L}^{+} W_{L}^{-} \rightarrow Z_{L} Z_{L}$ and $Z_{L} Z_{L} \rightarrow Z_{L} Z_{L}$. Standard Model backgrounds leading to $q q Z Z$ in the final state have been generated with COMPHEP, with cuts $p_{T}(q, Z)>15 \mathrm{GeV}, m_{Z Z}>500 \mathrm{GeV}, m_{q q}>200 \mathrm{GeV}$, with CTEQ5L structure functions and $Q=m_{Z}$. The process was implemented in PYTHIA as an external process. The Higgs mass was set at $100 \mathrm{GeV}$, so that the contribution from longitudinal vector boson scattering was negligible in this background. With these conditions, the SM cross-section is $69.4 \mathrm{fb}$. Other backgrounds considered were $q q \rightarrow Z Z$, with cut $m_{Z Z}>500 \mathrm{GeV}$ and cross-section $8.66 \mathrm{fb}$. The background $g g \rightarrow Z Z$ was not included, but is expected to contribute about one third of the $q \bar{q}$ fusion process [29].

The analysis requires the presence of four isolated leptons with transverse momenta greater than $30 \mathrm{GeV}$, and with two-lepton invariant masses compatible with coming from $Z$ bosons. Forward jet tagging was applied by requiring the presence of one forward and one backward jet with energies greater than $400 \mathrm{GeV}$. No central jet veto was imposed, as this is not needed to reject the main backgrounds. The expected signal and background for a resonance of mass $750 \mathrm{GeV}$, corresponding to $7 \alpha_{4}+11 \alpha_{5}=0.063$, is shown in Fig. 12 for an integrated luminosity of $3000 \mathrm{fb}^{-1}$. Such a process would not be observable at the nominal LHC.

\subsection{3 $W_{L}^{+} W_{L}^{+}$}

Non-resonant production of vector boson pairs at high mass represents a challenge at LHC because the background needs to be very well understood.

The production of $W^{+} W^{+}$pairs has no contribution from $q \bar{q}$ fusion. Two models have been considered: 


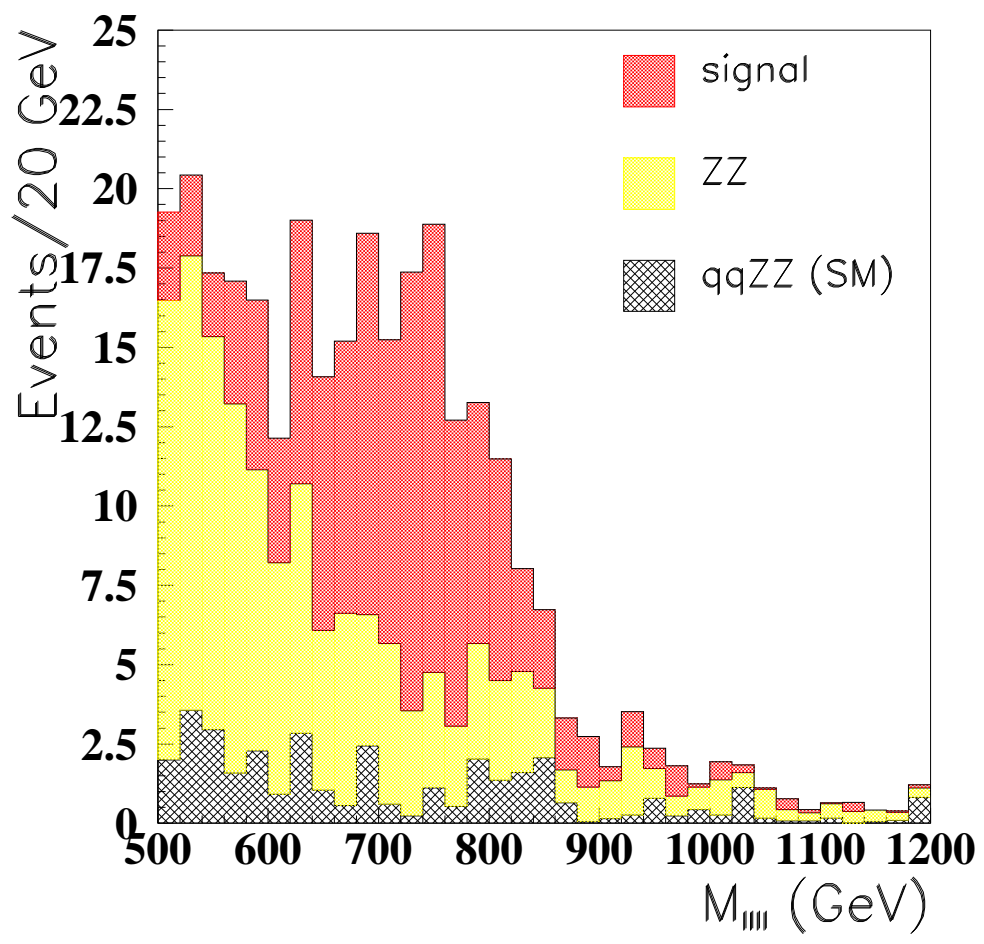

Fig. 12: Expected signal and background at the SLHC $\left(3000 \mathrm{fb}^{-1}\right)$ for a scalar resonance of mass $750 \mathrm{GeV}$ decaying into four leptons.

- A Higgs boson of mass $1 \mathrm{TeV}$ (as a reference point).

- WW production with K-matrix unitarization.

Backgrounds arise from gauge boson pairs produced by electroweak and gluon exchange diagrams [1]. Here only the most promising $\ell^{+} \ell^{+} \nu \nu$ final state, arising from same-sign $W^{+} W^{+}$production, is discussed. Selection cuts were applied on the transversa momenta of both leptons, on their azimuthal angle and on the event missing transverse energy [1]. Two forward tagging jets with energies above 400 (600) $\mathrm{GeV}$ were required for the LHC (SLHC) scenario, and events were rejected if there was a central jet with transverse momentum greater than 40 (60) GeV.

Table 10 shows the number of signal and background events expected, and the signal significance, for the LHC, the SLHC and (for comparison) for a machine with $\sqrt{s}=28 \mathrm{TeV}$.

In spite of the increase in statistical significance at the SLHC compared to the standard LHC, it should be noticed that extraction of a convincing signal in this channel will not be easy, because the shapes of the background and of the signal are similar.

\subsection{Top-quark physics}

Given the large top quark cross-section, most of the top physics programme should be completed during the first few years of LHC operation [32]. In particular, the $t \bar{t}$ and the single-top production cross-sections should be measured more precisely than the expected theoretical uncertainties, and the determination of the top mass should reach an uncertainty (dominated by systematics) of $\sim 1 \mathrm{GeV}$, beyond which more data offer no obvious improvement.

There is however one issue in top physics, namely rare decays, that the LHC can only address with limited statistics. While most of the rare decays expected in the SM are beyond any possible reach, there is a large class of theories beyond the SM where branching fractions for decays of top quarks induced 
Table 10: Expected numbers of reconstructed events above an invariant mass of $600 \mathrm{GeV}$ (for $\sqrt{s}=14 \mathrm{TeV}$ ) and $800 \mathrm{GeV}$ (for $\sqrt{s}=28 \mathrm{TeV}$ ) for models with a strongly-coupled Higgs sector and for the background. The significance was computed as $S / \sqrt{S+B}$.

\begin{tabular}{|c|c|c|c|c|}
\hline Model & $\begin{array}{c}300 \mathrm{fb}^{-1} \\
14 \mathrm{TeV}\end{array}$ & $\begin{array}{c}3000 \mathrm{fb}^{-1} \\
14 \mathrm{TeV}\end{array}$ & $\begin{array}{c}300 \mathrm{fb}^{-1} \\
28 \mathrm{TeV}\end{array}$ & $\begin{array}{c}3000 \mathrm{fb}^{-1} \\
28 \mathrm{TeV}\end{array}$ \\
\hline Background & 7.9 & 44 & 20 & 180 \\
\hline K-matrix Unitarization & 14 & 87 & 57 & 490 \\
Significance & 3.0 & 7.6 & 6.5 & 18.9 \\
\hline Higgs, 1 TeV & 7.2 & 42 & 18 & 147 \\
Significance & 1.8 & 4.5 & 2.9 & 8.1 \\
\hline
\end{tabular}

by flavour-changing neutral currents (FCNC) be as large as $10^{-5}-10^{-6}$. Studies documented in [32] indicate that the data which can be collected with a luminosity of $10^{34} \mathrm{~cm}^{-2} \mathrm{~s}^{-1}$ are not sufficient to explore these models.

Three possible FCNC decays have been investigated:

$$
\begin{array}{ll}
t \rightarrow q \gamma, & q=u \text { or } c \\
t \rightarrow q Z, & q=u \text { or } c \\
t \rightarrow q g, & q=u \text { or } c
\end{array}
$$

For each channel the number of signal events was evaluated for the reference value of

$$
\mathrm{BR}_{\mathrm{def}}(t \rightarrow(u+c) V)=1.0 \times 10^{-3}, \quad V=\gamma, Z, g
$$

The "reachable" branching ratio for $t \rightarrow q V$ decay was estimated as follows [33]:

$$
\frac{S}{\sqrt{S+B}+\sqrt{B}} \geq \frac{3}{2} \sigma, \quad(\mathrm{CL}=99 \%)
$$

where $S$ and $B$ stand for the numbers of signal and background events, respectively. The considered background processes include:

- $t \bar{t}(\sigma=830 \mathrm{pb})$

- $W(\rightarrow e, \mu)+$ jets $\left(\sigma \sim 7500 \mathrm{pb}\right.$ for $\left.p_{T, W}>20 \mathrm{GeV}\right)$

- $W W+W Z+Z Z(\sigma=110 \mathrm{pb})$

- $W \gamma(\sigma=17.3 \mathrm{pb})$

- single top (generated with TopRex [34]) $(\sigma=240 \mathrm{pb})$

All $b$-tagged jets should have $|\eta|<2.5$. We considered three cases for the $b$-tagging efficiency:

- An ideal case, where jets from $b, c$ and light quarks are identified and distinguished with $100 \%$ efficiency.

- A realistic case, based on a CMS simulation valid for a luminosity of $10^{34} \mathrm{~cm}^{-2} \mathrm{~s}^{-1}$ where the $b$-tagging efficiency is $\epsilon_{b} \approx 60 \%$, the mistagging probability for $c$-jets $\approx 10 \%$, and the mistagging probability for light-quark and gluon jets $\approx(1-2) \%$.

- A pessimistic case, in which only semileptonic muon decays of the $b$-quarks can be used. In particular, we require $p_{T}(\mu) \geq 20 \mathrm{GeV}$ for non-isolated muons carrying at least $60 \%$ of the jet energy, and having a $p_{T}$ relative to the jet axis larger than $700 \mathrm{MeV}$. This algorithm leads to a b-tagging efficiency $\epsilon_{b}=6.4 \%$.

$\mathbf{t} \rightarrow \mathbf{q} \gamma$

We consider $\left(\gamma+\ell^{ \pm}+\geq 2\right.$ jets $)$ final states, with the following cuts: 
Table 11: For $t \rightarrow q \gamma$ decays, the achievable branching ratio (in units of $10^{-5}$ ) at the LHC and SLHC for different $b$-tagging hypotheses (see text).

\begin{tabular}{|l||c|c|c|}
\hline$b$-tagging & ideal & real. & $\mu$-tag \\
\hline $600 \mathrm{fb}^{-1}$ & 0.48 & 0.88 & 3.76 \\
\hline $6000 \mathrm{fb}^{-1}$ & 0.14 & 0.26 & 0.97 \\
\hline
\end{tabular}

Table 12: For $t \rightarrow q g$ decays, the achievable branching ratio (in units of $10^{-5}$ ) at the LHC and SLHC for different $b$-tagging hypotheses (see text).

\begin{tabular}{|l||c|c|c|}
\hline$b$-tagging & ideal & real. & $\mu$-tag \\
\hline $600 \mathrm{fb}^{-1}$ & 22.3 & 60.8 & 210. \\
\hline $6000 \mathrm{fb}^{-1}$ & 7.04 & 19.2 & 66.2 \\
\hline
\end{tabular}

- One isolated photon, with $E_{T} \geq 75 \mathrm{GeV}$ and $\left|\eta_{\gamma}\right| \leq 2.5$.

- One isolated lepton, with $p_{T} \geq 20 \mathrm{GeV}$ and $\left|\eta_{\ell}\right| \leq 2.5$.

- two or more jets with $E_{T j} \geq 30 \mathrm{GeV}$ and $\left|\eta_{j}\right| \leq 4.5$. No third jet with $E_{T j} \geq 50 \mathrm{GeV}$. One of the jets should be $b$-tagged.

- One pairing of the jets, the $\gamma$ and the reconstructed semileptonic top such that:

$$
\left|M\left(j_{1}+\gamma\right)-m_{t}\right| \leq 15 \mathrm{GeV} \quad\left|M(\mathrm{~b}-j e t+W)-m_{t}\right| \leq 25 \mathrm{GeV}
$$

The results are given in Table 11 .

$\mathbf{t} \rightarrow \mathbf{q g}$

We consider $\left(\ell^{ \pm}+\geq 3\right.$ jets) final states, with the following cuts:

- One isolated lepton, with $p_{T} \geq 20 \mathrm{GeV}$ and $\left|\eta_{\ell}\right| \leq 2.5$.

- $N_{\text {jets }}=3$, with $E_{T j} \geq 50 \mathrm{GeV}$ and $\left|\eta_{j}\right| \leq 4.5$. Only one of the three jets should be $b$-tagged.

- At least one combination such that $\left|M(\mathrm{~b}-j e t+W)-m_{t}\right| \leq 25 \mathrm{GeV}$ and $\left|M\left(j_{1}+j_{2}\right)-m_{t}\right| \leq$ $25 \mathrm{GeV}$.

The results are given in Table 12 .

$\mathbf{t} \rightarrow \mathbf{q} \mathbf{Z}$

We consider ( $3 \ell^{ \pm}+\geq 2$ jets) final states, with the following cuts:

- Three isolated leptons with $p_{T} \geq 20 \mathrm{GeV}$ and $\left|\eta_{\ell}\right| \leq 2.5$.

- $N_{\text {jets }} \geq 2$, with $E_{T j} \geq 50 \mathrm{GeV}$ and $\left|\eta_{j}\right| \leq 4.5$. Only one of the jets should be $b$-tagged.

- At least one combination with $\left|M(\mathrm{~b}-j e t+W)-m_{t}\right| \leq 25 \mathrm{GeV}$ and $\left|M(Z+j)-m_{t}\right| \leq 25 \mathrm{GeV}$.

The results are given in Table 13 .

With a detector performance comparable to that expected at $10^{34} \mathrm{~cm}^{-2} \mathrm{~s}^{-1}$, the SLHC should enhance by a large factor the sensitivity to FCNC top decays. In the case of $t \rightarrow Z q$, in particular, the improvement is almost linear with the luminosity, thanks to the very low background level. Branching ratios of order $10^{-6}$ are achievable, which are of interest for some theories beyond the Standard Model,

Table 13: For $t \rightarrow q Z$ decays, the achievable branching ratio (in units of $10^{-5}$ ) at the LHC and SLHC for different $b$-tagging hypotheses (see text).

\begin{tabular}{|l||c|c|c|}
\hline$b$-tagging & ideal & real. & $\mu$-tag \\
\hline $600 \mathrm{fb}^{-1}$ & 0.46 & 1.1 & 83.3 \\
\hline $6000 \mathrm{fb}^{-1}$ & 0.05 & 0.11 & 8.3 \\
\hline
\end{tabular}




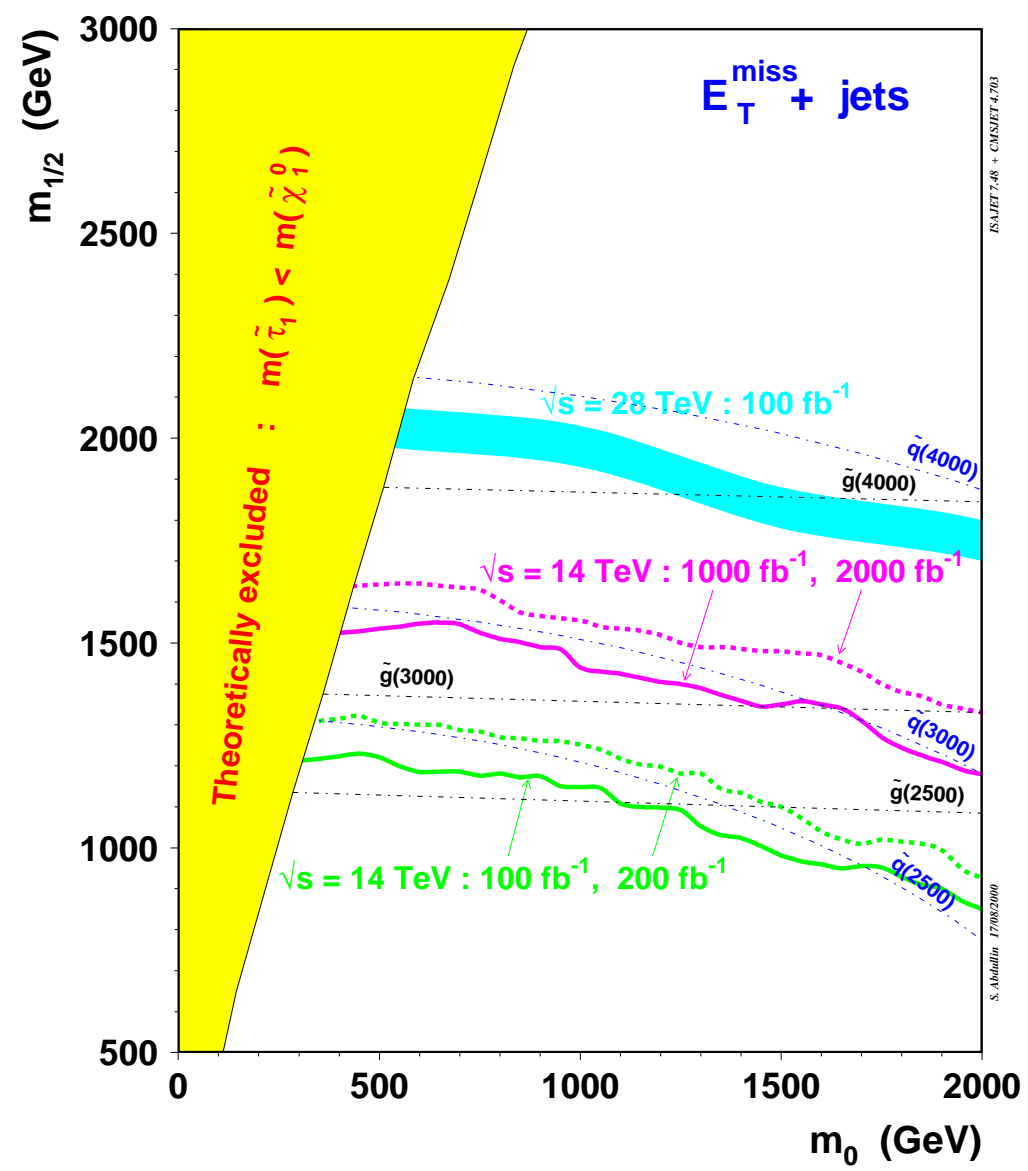

Fig. 13: Expected $5 \sigma$ discovery contours in the mSUGRA plane $m_{0}$ versus $m_{1 / 2}$ for $A_{0}=0, \tan \beta=10$ and $\mu<0$. The various curves show the potential of the CMS experiment at the standard LHC (for luminosities of $100 \mathrm{fb}^{-1}$ and $200 \mathrm{fb}^{-1}$ ), at the SLHC (for $1000 \mathrm{fb}^{-1}$ and $2000 \mathrm{fb}^{-1}$ ), and (for comparison) at a machine with a centre-of-mass energy of $28 \mathrm{TeV}$.

as discussed in [32]. Loss of the ability to tag $b$-quarks with a secondary vertex technique would however downgrade the sensitivity to such a level that no gain could be obtained from SLHC data compared to the standard LHC.

\subsection{Supersymmetry}

If Supersymmetry is connected to the hierarchy problem, it is expected that sparticles will be sufficiently light that at least some of them will be observed at the LHC. However it is not possible to set a rigorous bound on the sparticle masses, and it may well be that the heaviest part of the SUSY spectrum (usually squarks and gluinos) is missed at the standard LHC.

The LHC discovery potential for squarks and gluinos, in several energy and luminosity scenarios, is summarised in Fig. 13. The various contours were derived within the framework of minimal Supergravity models (mSUGRA), and are shown as a function of the universal scalar mass $m_{0}$ and of the universal gaugino mass $m_{1 / 2}$. They were obtained by looking for events with many high- $p_{T}$ jets and large missing transverse energy. This is the most typical and most model-independent signature for SUSY if R-parity is conserved.

It can be seen that a luminosity upgrade would extend the mass reach for squarks and gluinos from about 2.5 TeV (standard LHC) to about $3 \mathrm{TeV}$ (SLHC). This performance does not require major 

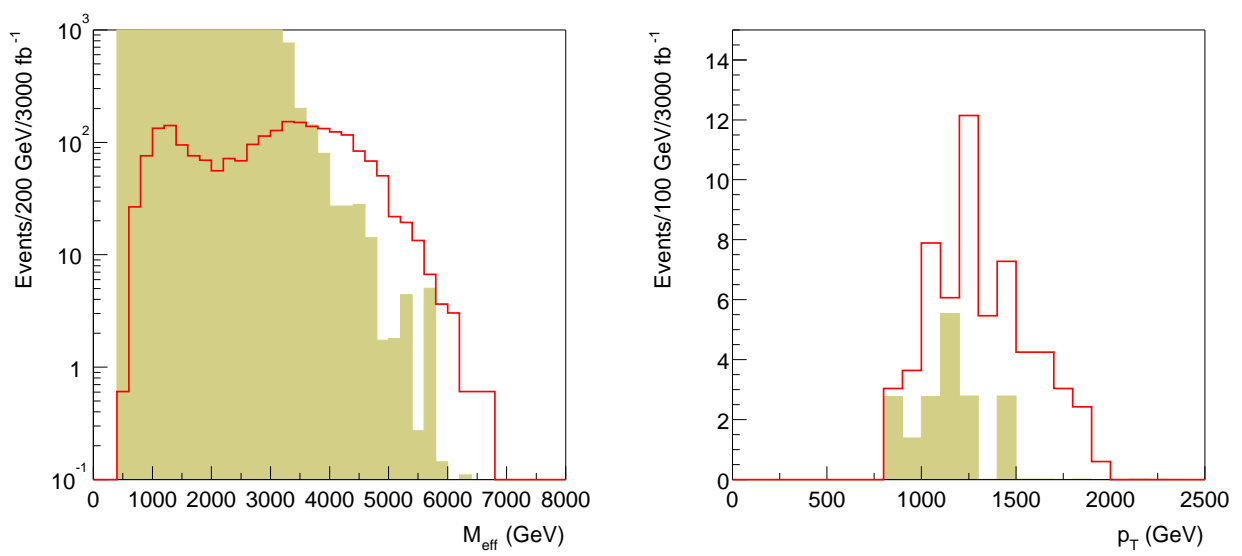

Fig. 14: For Point K, the distributions of the effective mass (left) and of the $p_{T}$ of the hardest jet from a sample of 2 jets + $E_{T}^{\text {miss }}$ events (right). Solid: signal. Shaded: SM background.

detector upgrades because these inclusive searches are based mainly on calorimetric measurements of high- $p_{T}$ jets and large missing transverse energy. On the other hand, reconstruction of more exclusive decay chains which may be rate-limited at the LHC, such as some cascade decays of heavy gauginos, could become possible at the SLHC provided the full detector functionality is preserved. To illustrate this case, two points (here called $\mathrm{K}$ and $\mathrm{H}$ ) of the mSUGRA parameter space have been studied in some detail [35]. These points were taken from a recently-proposed set of benchmark points [36], that satisfy existing bounds including dark matter constraints and results from direct searches at LEP. Three of these points $(\mathrm{F}, \mathrm{H}, \mathrm{K})$, where the squark and gluino masses exceed $2 \mathrm{TeV}$, might benefit from a luminosity upgrade 2 , because the expected event rates are small at the LHC and therefore detailed SUSY studies will not be possible.

Point $\mathrm{K}$ has gluino and squark masses slightly above $2 \mathrm{TeV}$. Squark pair production dominates, and is followed by the decays $\tilde{q}_{L} \rightarrow \tilde{\chi}_{1}^{ \pm} q, \tilde{\chi}_{2}^{0} q$ and $\tilde{q}_{R} \rightarrow \tilde{\chi}_{1}^{0} q$. The signal can inclusively be observed on top of the background by using for instance the distribution of the effective mass, defined as

$$
M_{\text {eff }}=E_{T}^{\text {miss }}+\sum_{\text {jets }} E_{T, j e t}+\sum_{\text {leptons }} E_{T, l e p t o n}
$$

where the sum runs over all jets with $E_{T}>50 \mathrm{GeV}$ and $|\eta|<5.0$ and isolated leptons with $E_{T}>15$ $\mathrm{GeV}$ and $|\eta|<2.5$. Events were selected with at least two jets with $p_{T}>0.1 M_{\text {eff }}, E_{T}^{\text {miss }}>0.3 M_{\text {eff }}$, $\Delta \phi\left(j_{1}, E_{T}^{m i s}\right)<\pi-0.2$, and $\Delta \phi\left(j_{1}, j_{2}\right)<2 \pi / 3$. The distributions in $M_{e f f}$ for signal and background are shown in Fig. 14. The signal emerges from the background at large values of $M_{\text {eff }}$. For an integrated luminosity of $3000 \mathrm{fb}^{-1}$, a signal of 500 events should be observed on top of a background of 100 events for $M_{\text {eff }}>4000 \mathrm{GeV}$. These rates are sufficiently large that a discovery could be made already at the LHC.

Production of $\tilde{q}_{R} \tilde{q}_{R}$ followed by the decay of each squark to $q \tilde{\chi}_{1}^{0}$ gives a di-jet signal accompanied by missing $E_{T}$. In order to extract this signature from the Standard Model background, hard cuts on the jets and $E_{T}^{\text {miss }}$ are needed. Events were required to have two jets with $p_{T}>700 \mathrm{GeV}, E_{T}^{\text {miss }}>600 \mathrm{GeV}$, and $\Delta \phi\left(j_{1}, j_{2}\right)<0.8$. The resulting distribution of the $p_{T}$ of the hardest jet is shown in Fig. 14. Only a few events survive with $3000 \mathrm{fb}^{-1}$, hence this exclusive channel is not observable at the standard LHC. The transverse momentum of the hardest jet is sensitive to the $\tilde{q}_{R}$ mass [1]]. The mass determination will be limited by the available statistics.

Since the decay $\tilde{\chi}_{2}^{0} \rightarrow \tilde{\chi}_{1}^{0} h$ is dominant at Point K, Higgs particles should be found in the decay $\tilde{q}_{L} \rightarrow \tilde{\chi}_{2}^{0} q$, followed by $\tilde{\chi}_{2}^{0} \rightarrow \tilde{\chi}_{1}^{0} h$. The Higgs signal should be observed as a peak in the $b \bar{b}$ invariant

\footnotetext{
${ }^{2}$ Point $\mathrm{M}$ is beyond the sensitivity even of a SLHC, given that the squark and gluino masses are above $3 \mathrm{TeV}$.
} 

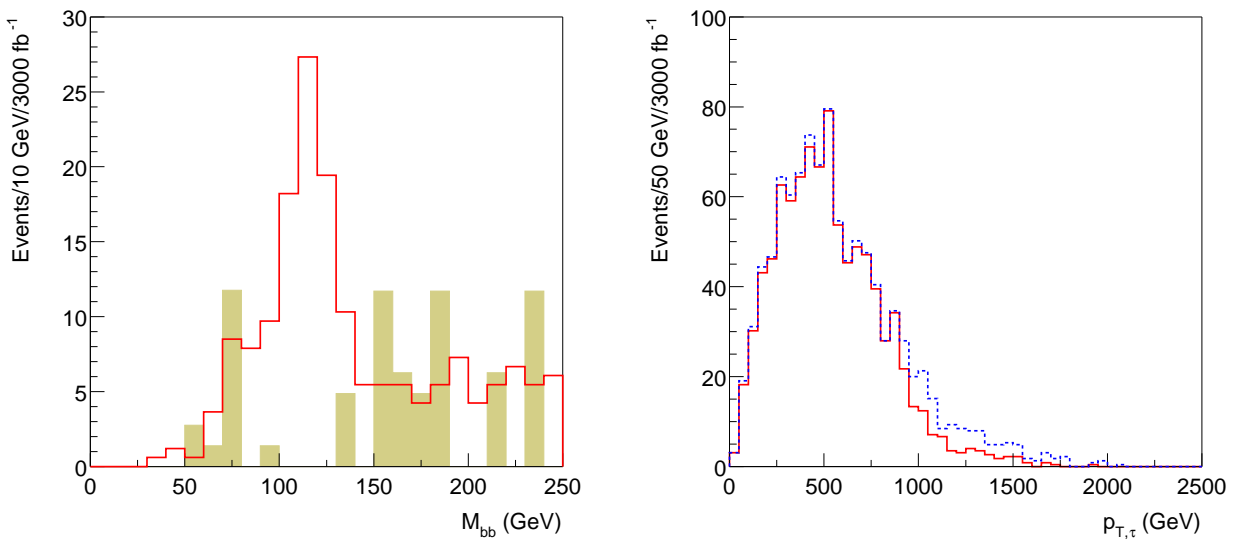

Fig. 15: Left: the $b \bar{b}$ invariant mass distribution for Point K (solid: signal; shaded: SM background). Right: the $p_{T}$ distribution of $\tilde{\tau}_{1}$ for Point $\mathrm{H}$ (dashed: all $\tilde{\tau}_{1}$; solid: $\tilde{\tau}_{1}$ reaching the ATLAS Muon Spectrometer with a delay $\Delta t>7 \mathrm{~ns}$ ).

mass distribution. It is therefore essential that $b$-jets can be tagged with good efficiency and excellent rejection against light-quark jets. There is a large background from $t \bar{t}$ production that must be overcome using topological cuts. Events were selected to have at least three jets with $p_{T}>600,300,100 \mathrm{GeV}$, $E_{T}^{\text {miss }}>400 \mathrm{GeV}, M_{\text {eff }}>2500 \mathrm{GeV}, \Delta \phi\left(j_{1}, E_{T}^{\text {miss }}\right)<0.9$, and $\Delta \phi\left(j_{1}, j_{2}\right)<0.6$. The $b \bar{b}$ invariant mass distribution is shown in Fig. 15 assuming a $b$-tagging efficiency of $60 \%$ and a rejection of $\sim 100$ against light-quark jets.

Point $\mathrm{H}$ is characterised by squark and gluino masses above $2.5 \mathrm{TeV}$ and by almost degenerate $\tilde{\tau}_{1}$ and $\tilde{\chi}_{1}^{0}$ masses. In this particular case, $\tilde{\tau}_{1} \nrightarrow \tilde{\chi}_{1}^{0} \tau$, so $\tilde{\tau}_{1}$ must decay by second order weak processes, $\tilde{\tau}_{1} \rightarrow \tilde{\chi}_{1}^{0} e \bar{\nu}_{e} \nu_{\tau}$, with a long lifetime. The dominant SUSY rates arise from the strong production of squark pairs, with $\tilde{q}_{L} \rightarrow \tilde{\chi}_{1}^{ \pm} q, \tilde{\chi}_{2}^{0} q$ and $\tilde{q}_{R} \rightarrow q \tilde{\chi}_{1}^{0}$. The staus which are produced from cascade decays of the gauginos traverse the detector with a signal similar to a "heavy muon". The $p_{T}$ spectrum of these quasistable $\tilde{\tau}_{1}$ is shown in Fig. 15. The ATLAS Muon Spectrometer [1] has a resolution of about $0.7 \mathrm{~ns}$ for the time of flight measurement. The spectrum for staus reaching the muon chambers with a time delay $\Delta t>7 \mathrm{~ns}(10 \sigma)$ is also shown in Fig. 15. This signal could be observed with $\sim 300 \mathrm{fb}^{-1}$. The mass of the stable stau can be determined by combining a momentum measurement with a time of flight measurement in the Muon Spectrometer. Studies of such quasi-stable particles at somewhat smaller masses carried out with simulations of the ATLAS detector showed a mass resolution of approximately $3 \%$ given sufficient statistics [1]. A precision of this order should be achieved for Point $\mathrm{H}$ with $3000 \mathrm{fb}^{-1}$.

The stable $\tilde{\tau}_{1}$ signature is somewhat exceptional. Therefore other signatures that would be present if the stau decayed inside the detector were examined. Events were selected by requiring at least two jets with $p_{T}>0.1 M_{\text {eff }}, E_{T}^{\text {miss }}>0.3 M_{\text {eff }}, \Delta \phi\left(j_{1}, E_{T}^{\text {miss }}\right)<\pi-0.2$, and $\Delta \phi\left(j_{1}, j_{2}\right)<2 \pi / 3$. The $M_{\text {eff }}$ distributions after these cuts show that the number of events in the region where $S / B>1$ is of order 30 for $3000 \mathrm{fb}^{-1}$. Di-leptons arise from the cascade decay $\tilde{q}_{L} \rightarrow q \tilde{\chi}_{2}^{0} \rightarrow q \ell^{+} \ell^{-} \tilde{\chi}_{1}^{0}$. The di-lepton invariant mass distributions should have a kinematic end-point corresponding to this decay. Figure 16 shows the distribution for same-flavor and different-flavor lepton pairs. Events were required to have $M_{\text {eff }}>3000 \mathrm{GeV}, E_{T}^{\text {miss }}>0.2 M_{\text {eff }}$, and two isolated opposite-sign leptons with $E_{T}>15 \mathrm{GeV}$ and $|\eta|<2.5$. The end-point structure may be observable, although it should be noted that the background has large errors as only three generated events passed the cuts. The edge comes mainly from $\tilde{\chi}_{2}^{0} \rightarrow \tilde{\ell}_{L}^{ \pm} \ell^{\mp}$, which has a branching ratio of $15 \%$ per flavor. This gives an end-point at

$$
\sqrt{\frac{\left(M_{\tilde{\chi}_{2}^{0}}^{2}-M_{\tilde{\ell}_{L}}^{2}\right)\left(M_{\tilde{\ell}_{L}}^{2}-M_{\tilde{\chi}_{1}^{0}}^{2}\right)}{M_{\tilde{\ell}_{L}}^{2}}}=447.3 \mathrm{GeV}
$$



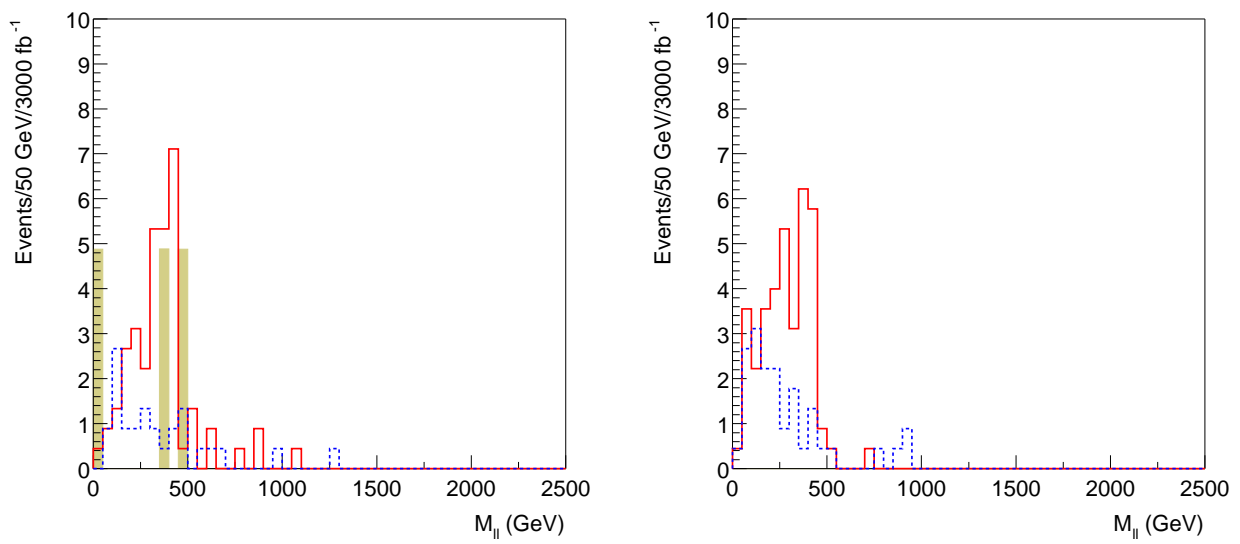

Fig. 16: For Point H, the $M_{\ell \ell}$ distribution for all events (left) and events containing a stable stau (right). Solid: same-flavour lepton pairs. Dashed: different-flavour lepton pairs. Shaded: SM background.

Table 14: $Z^{\prime}$ production cross-section at the LHC times branching ratio into electron pairs and $Z^{\prime}$ width, as a function of mass.

\begin{tabular}{|c|c|c|c|c|c|c|}
\hline$Z^{\prime}$ mass $(\mathrm{TeV})$ & 1 & 2 & 3 & 4 & 5 & 6 \\
\hline$\sigma\left(Z^{\prime} \rightarrow e^{+} e^{-}\right)(f b)$ & 512 & 23.9 & 2.5 & 0.38 & 0.08 & 0.026 \\
\hline$\Gamma_{Z^{\prime}}(\mathrm{GeV})$ & 30.6 & 62.4 & 94.2 & 126.1 & 158.0 & 190.0 \\
\hline
\end{tabular}

consistent with the observed end-point in Fig. 16. Obviously this distribution does not distinguish $\tilde{\ell}_{L}$ and $\tilde{\ell}_{R}$.

If the stable stau signature is used, then the situation improves considerably. The di-lepton mass for events containing a $\tilde{\tau}_{1}$ with a time delay in the ATLAS Muon Spectrometer in the range $7<\Delta t<21.5 \mathrm{~ns}$ is shown in Fig. 16. Since $\Delta t>10 \sigma$, the Standard Model background is expected to be negligible. A measurement of the end-point should be possible at the SLHC.

\subsection{New gauge bosons}

The potential of the SLHC for the discovery of additional heavy gauge bosons has been studied by considering a $Z^{\prime}$ with $Z$-like couplings to leptons and quarks. The $Z^{\prime}$ production cross-section times branching ratio into electron pairs $\sigma\left(Z^{\prime} \rightarrow e^{+} e^{-}\right)$and the $Z^{\prime}$ width are shown in Table 14 as a function of mass.

The study is based on the CMS detector performance and the result is then extrapolated to the case of two experiments. The analysis takes into account acceptance, reconstruction efficiency and resolution for muons and electrons. The expected pile-up noise at $10^{35} \mathrm{~cm}^{-2} \mathrm{~s}^{-1}$ is also included, as well as saturation effects in the CMS crystal calorimeter.

The $Z^{\prime}$ mass is reconstructed conservatively without correcting for internal photon radiation. This leads to some tails in the mass spectra. The expected number of signal and background events is calculated from the gaussian part of these spectra. The background is found to be small, i.e. $\simeq 2 \%$ from Drell-Yan production and less than $1 \%$ from $t \bar{t}$. The expected number of signal events for two experiments is shown in Fig. 17. Assuming that a discovery can be claimed if the number of observed events is at least ten, the LHC discovery reach improves from $\sim 5.3 \mathrm{TeV}$ (standard LHC, $600 \mathrm{fb}^{-1}$ ) to $\sim 6.5 \mathrm{TeV}$ (SLHC, $6000 \mathrm{fb}^{-1}$ ). For comparison, a machine with $\sqrt{s}=28 \mathrm{TeV}$ running at $10^{34} \mathrm{~cm}^{-2} \mathrm{~s}^{-1}$ would extend the mass reach of the standard LHC by $50 \%$ [ $₫$.

It should also be noted that discovery will be mainly based on the electron final state, which provides the best mass resolution, whereas measurements of couplings and asymmetries will use both lepton channels. 


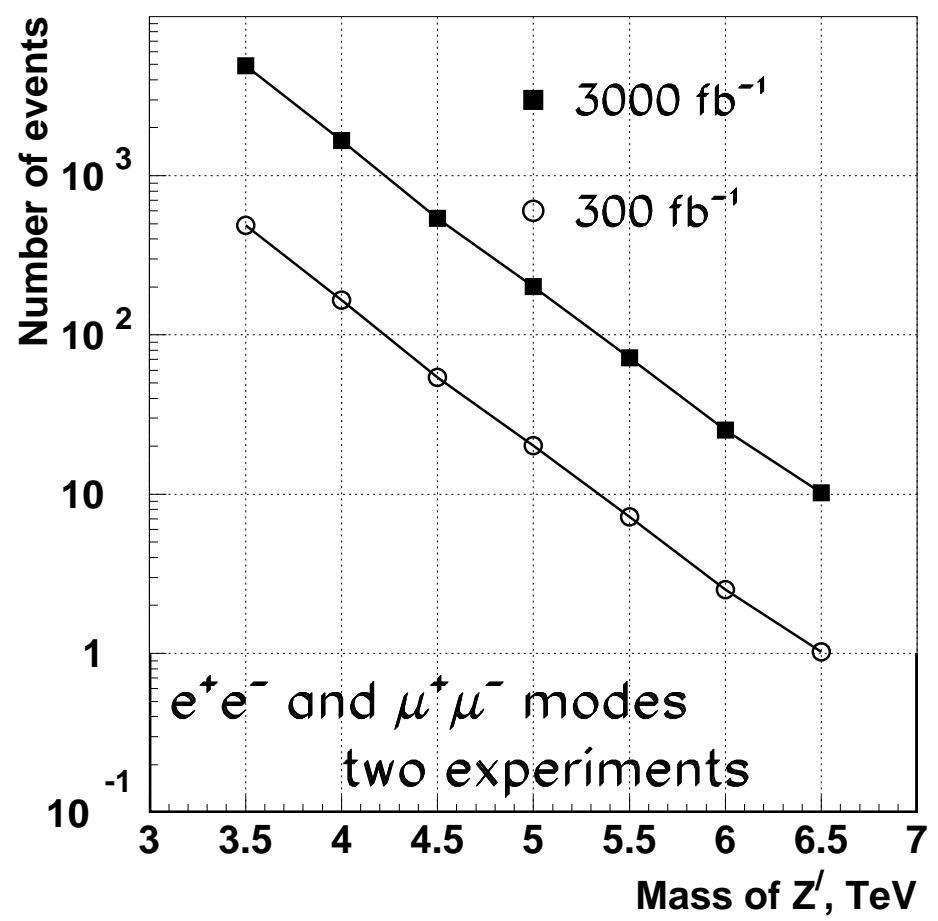

Fig. 17: Expected number of $Z^{\prime} \rightarrow \mu^{+} \mu^{-}, e^{+} e^{-}$events in both experiments for integrated luminosities of $300 \mathrm{fb}^{-1}$ per experiment and $3000 \mathrm{fb}^{-1}$ per experiment.

\subsection{Extra-dimensions}

Theories with large extra-dimensions, which aim at solving the hierarchy problem by allowing the gravity scale to be close to the electroweak scale, have recently raised a lot of interest. They predict new phenomena in the $\mathrm{TeV}$ energy range, which can therefore be tested at present and future colliders. Several models and signatures have been considered in the study presented here. They are discussed below.

\subsubsection{Direct graviton production in ADD models}

In these models [37], the extra-dimensions are compactified to the sub-millimiter size and only gravity is allowed to propagate in them, whereas the SM fields are confined to a 4-dimensional world. Gravitons in the extra-dimensions occupy energy/mass levels which are separated by very small splittings, and therefore give rise to a continuous tower of massive particles ('Kaluza-Klein (KK) excitations'). The presence of additional dimensions can therefore produce new phenomena involving gravitons, such as direct graviton production at high energy colliders.

The most sensitive channel at the LHC should be the associated production of KK gravitons with a quark or a gluon. The resulting signature is an energetic jet plus missing transverse energy, since the gravitons escape detection. The cross-section depends on two parameters, the gravity scale $M_{D}$ and the number of extra-dimensions $\delta$, and decreases with increasing values of both $M_{D}$ and $\delta$. The background is dominated by the final state $Z(\rightarrow \nu \nu)+$ jets.

The discovery potentials of the LHC and SLHC are compared in Fig. 18. It can be seen that a factor of ten in luminosity would improve the LHC mass reach by typically $30 \%$. Major detector upgrades are not crucial for this physics, since the search is based on events with jets and missing energy in the $\mathrm{TeV}$ range. For comparison, doubling the LHC energy but keeping the instantaneous luminosity of $10^{34} \mathrm{~cm}^{-2} \mathrm{~s}^{-1}$ would approximately double the reach in $M_{D}$ for any value of $\delta$ 沛. 


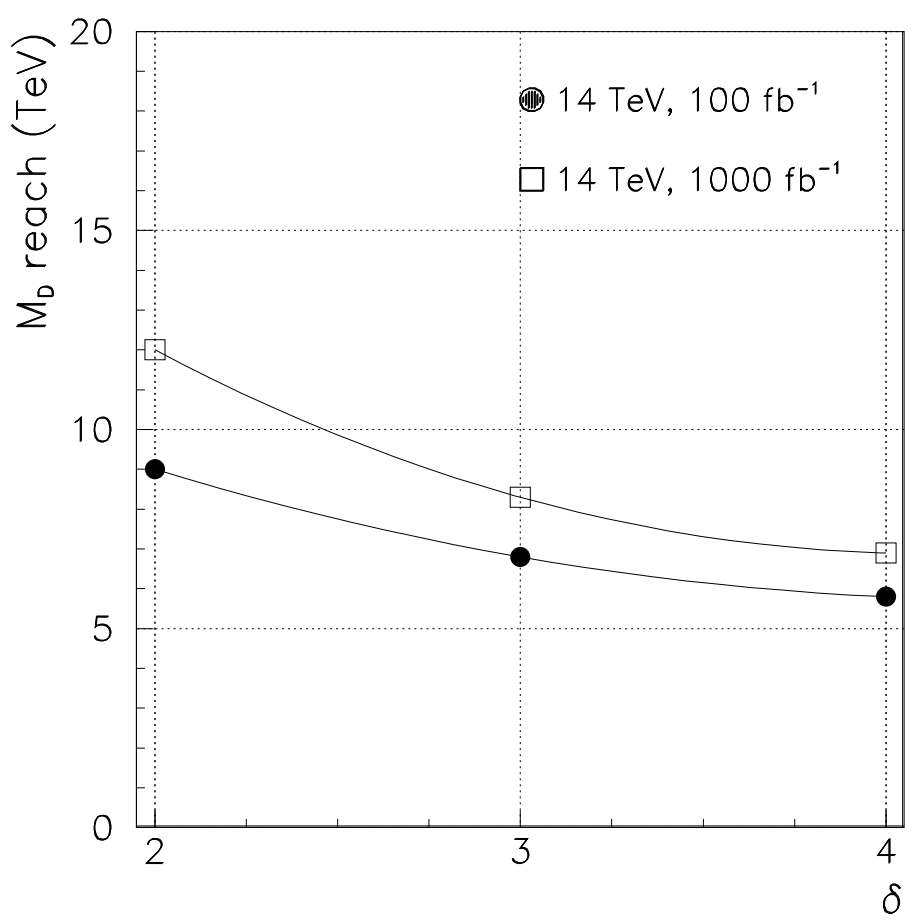

Fig. 18: Expected $5 \sigma$ discovery reach on the gravity scale $M_{D}$ as a function of the number of extra-dimensions $\delta$ in ATLAS in the framework of ADD models, for $100 \mathrm{fb}^{-1}$ (LHC) and $1000 \mathrm{fb}^{-1}$ (SLHC).

\subsubsection{Virtual graviton exchange in ADD models}

Virtual KK gravitons can also be exchanged between incoming and outgoing SM particles in high-energy collisions, thereby leading to modifications of the cross-section and angular distributions compared to the SM expectations. Since graviton effects are enhanced at high energy, due to the large number of accessible Kaluza-Klein excitations, such manifestations of Extra-dimensions are expected at large invariant mass and $p_{T}$ of the particles in the final state. Drell-Yan and two-photon production are among the most sensitive channels at high-energy colliders. Using these channels, it was found that the reach in the gravity scale $M_{D}$ for $\delta=3$ increases from $\sim 8 \mathrm{TeV}\left(100 \mathrm{fb}^{-1}\right.$, standard LHC) to $11.7 \mathrm{TeV}$ (3000 $\mathrm{fb}^{-1}, \mathrm{SLHC}$ ).

\subsubsection{Resonance production in Randall-Sundrum models}

In the Extra-dimension scenario proposed by Randall and Sundrum [38] the hierarchy between the Planck and the electroweak scales is generated by an exponential function called "warp factor". This model predicts KK graviton resonances with both weak scale masses and couplings to matter. In its simplest form, with only one extra-dimension, two distinct branes (the TeV brane and the Planck brane), and with all of the SM fields living on the TeV brane, the Randall-Sundrum model has only two fundamental parameters: the mass of the first KK state $m_{1}$ and the parameter $c=k / M_{\overline{P L}}$, where $k$ is related to the curvature of the 5-dimensional space and $M_{\overline{P L}}$ is the effective Planck scale. The parameter $c$ governs the width of the resonances, and is expected to be not far from unity.

Direct production of Randall-Sundrum resonances $(p p \rightarrow G)$ can lead to spectacular signals, for instance in the clean di-lepton decay mode $(G \rightarrow \ell \ell)$. They should be observable already in the first years of LHC running if $m_{1}$ is in the range 1-3 TeV. In addition, their properties (e.g. their spin-2 nature) can be measured and should distinguish them from e.g. $Z^{\prime}$ production [39]. Figure 19 summarises 95\% C.L. exclusion limits in the plane $m_{1}$ versus $c$-parameter. It shows the present constraints from 


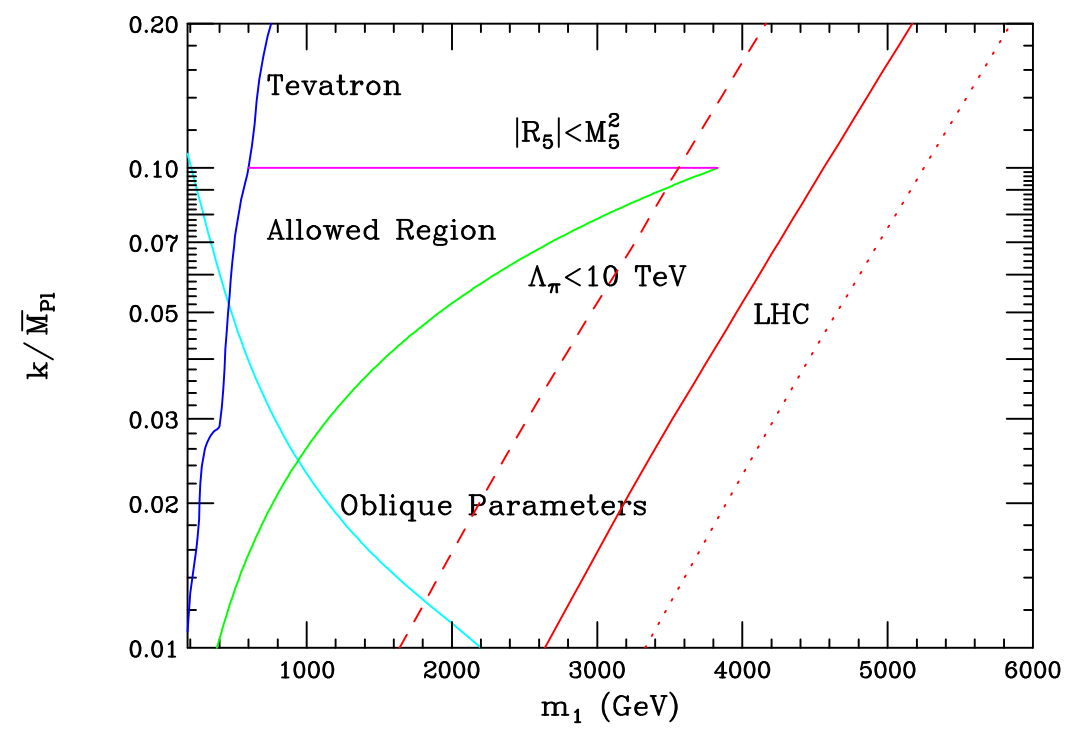

Fig. 19: 95\% C.L. exclusion limits in the plane $m_{1}$ versus $c$ (see text) for Randall-Sundrum graviton resonances decaying into electron or muon pairs. The triangular region labelled as 'allowed' represents the theoretically favoured domain. Here $M_{5}$ is the 5-dim Planck scale, $R_{5}$ is the 5-dim curvature invariant and $\Lambda_{\pi}$ is the inverse coupling strength of the KK gravitons. The bound $\left|R_{5}\right|<M_{5}^{2}$ is applied so that quantum gravity loop effects are small and we can treat the RS model classically. The dashed line and the full line show the LHC potential for integrated luminosities of $10 \mathrm{fb}^{-1}$ and $100 \mathrm{fb}^{-1}$ respectively, the dotted line shows the potential of the SLHC with $1000 \mathrm{fb}^{-1}$. The exclusion domains lie to the left of the lines. The present Tevatron limit is also indicated, as well as the region excluded by precision measurements of the electroweak oblique parameters $S, T, U$ at LEP.

LEP and Tevatron, as well as the potentials of the LHC and SLHC. The region labelled as "allowed" is theoretically favoured [40], although the rest of the plane is not strictly forbidden. It can be seen that the SLHC should extend the LHC reach by almost $1 \mathrm{TeV}$.

\subsubsection{Resonance production in $\mathrm{TeV}^{-1}$ scale Extra-dimensions}

In these models [41] only the fermion fields are confined to a 4-dimensional brane, whereas the SM gauge fields are allowed to propagate in a number of additional small extra-dimensions (compactification radius $\sim 1 \mathrm{TeV}^{-1}$ ), orthogonal to the brane. The most important phenomenological consequence is the predicted existence of KK excitations of the SM gauge bosons, $\gamma, Z, W$ and $g$. For simplicity, only the case of one extra-dimension is considered here. The model is completely specified by a single parameter $M_{c}$, the compactification scale, from which the masses $M_{n}$ of the KK excitations of the gauge bosons can be derived using the relation $M_{n}^{2}=\left(n M_{c}\right)^{2}+M_{0}^{2}$, where $M_{0}$ is the mass of the SM gauge boson. The couplings are the same as the corresponding SM couplings, scaled by a factor $\sqrt{2}$. Constraints from precision electroweak measurements give an approximate lower limit $M_{c}>4 \mathrm{TeV}$ [42].

The possibility of detecting the leptonic decays of the $K K$ excitations of the $\gamma$ and $Z$ bosons at the LHC and SLHC has been studied. The production of the gauge boson excitations, including interference terms and angular information, was performed using the full Breit-Wigner shape for the first two excited states [43], and a resummed expression for the higher states. The matrix elements were interfaced to PYTHIA, and the produced events were passed through a fast simulation of the ATLAS detector [7].

The main experimental issue in the study of these models is the measurement of leptons with very high transverse momenta. As an example, the ATLAS detector has been designed to measure leptons with $p_{T}$ up to $\sim 2-3 \mathrm{TeV}$. For electrons the main issue is the saturation of the dynamic range of the calorimeter electronics, which can be possibly compensated by a modification in the gain of the readout electronics. For muons, the momentum is obtained from the track curvature in the external Muon Spectrometer, and this measurement is very poor for transverse momenta above $4 \mathrm{TeV}$. 


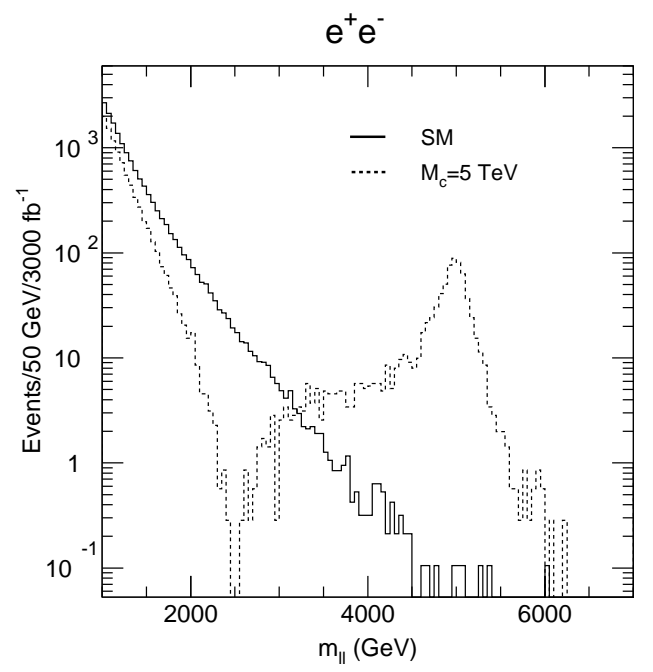

Fig. 20: Invariant mass distribution of $e^{+} e^{-}$pairs as expected from the Standard Model (full line) and from a gauge excitation model with $M_{c}=5 \mathrm{TeV}$ (dashed line). The histograms are normalised to $3000 \mathrm{fb}^{-1}$.

Events were selected by requiring two opposite-sign isolated leptons $(\ell=e, \mu)$ with $p_{T}>20 \mathrm{GeV}$, inside the rapidity range $|\eta|<2.5$ and with invariant mass $m\left(\ell^{+} \ell^{-}\right)>1000 \mathrm{GeV}$. In the absence of New Physics, approximately 18000 events survive these cuts for each lepton flavour and for an integrated luminosity of $3000 \mathrm{fb}^{-1}$. Figure 20 shows the expected two-lepton invariant mass spectrum for an Extradimension signal with $M_{c}=5 \mathrm{TeV}$ on top of the SM background. One can notice two structures. A peak centered around $M_{c}$, corresponding to the superposition of the first $\gamma$ and $Z$ resonances, and a suppression of the cross-section with respect to the SM expectation for masses below the resonance. This suppression is due to the negative interference between the SM gauge bosons and the whole tower of KK excitations, and is sizeable even for compactification scales well above those accessible form a direct detection of the mass peak.

The reach for the observation of a resonant peak in the $\ell^{+} \ell^{-}$invariant mass distribution can be obtained from Table 15, which gives the expected numbers of signal and background events in the electron and muon channels separately, for an integrated luminosity of $3000 \mathrm{fb}^{-1}$ and for different values of $M_{c}$. Using as a discovery criterion that at least ten events (summed over both lepton species and both experiments) be detected with two-lepton invariant mass above a given value, and that the signal statistical significance be $S / \sqrt{B}>5$, the reach should be $\sim 6 \mathrm{TeV}$ for $300 \mathrm{fb}^{-1}$ per experiment and $\sim 7.7 \mathrm{TeV}$ for $3000 \mathrm{fb}^{-1}$ per experiment (both experiments combined). It should be noticed that to achieve this result a good knowledge of the rate of background events at high masses, which could be affected by mismeasurements of the lepton momenta, is crucial. For $M_{c}=4 \mathrm{TeV}$ a few events from the second resonance at $8 \mathrm{TeV}$ could be observed, thereby hinting at the periodic structure of the mass spectrum of the resonances. For higher compactification scales, only the first KK excitation will most likely be accessible.

Sensitivity to a signal can also be obtained from the observation of off-resonance (negative) interference effects in the Drell-Yan mass spectrum (see Fig. 20). A detailed estimation requires a likelihood fit to the shape of the distribution. A simpler method was used to obtain the results reported here, which consists of evaluating the decrease in the number of events (compared to the SM expectation) inside a given $m\left(\ell^{+} \ell^{-}\right)$range, as a function of $M_{c}$. The statistical significance of the cross-section suppression is approximately given by $(N(t o t)-N(S M)) / \sqrt{N(S M)}$, where $N(t o t)$ is the total number of observed events and $N(S M)$ the expected number of events from the Standard Model. There are however uncertainties in the knowledge of the shape of the two-lepton invariant mass distribution, due to both instrumental effects (absolute energy scale, linearity), and theoretical effects (structure functions, 
Table 15: For an integrated luminosity of $3000 \mathrm{fb}^{-1}$ and one experiment, expected number of signal events in the peak region for different values of the mass of the lowest KK excitation $M_{c}$, and expected number of SM background events. The peak region is defined by a cut on the minimum $\ell^{+} \ell^{-}$invariant mass given in the second column. The results for electrons and muons are shown separately.

\begin{tabular}{|r|r|r|r|r|r|}
\hline$M_{c}(\mathrm{GeV})$ & Cut $(\mathrm{GeV})$ & Signal $(e)$ & Signal $(\mu)$ & Background $(e)$ & Background $(\mu)$ \\
\hline 4000 & 3000 & 5160 & 4680 & 44 & 54 \\
5000 & 4000 & 690 & 600 & 4.5 & 6.6 \\
5500 & 4000 & 270 & 240 & 4.5 & 6.6 \\
6000 & 4500 & 99 & 84 & 1.5 & 3 \\
7000 & 5000 & 13.5 & 11.4 & 0.45 & 0.5 \\
8000 & 6000 & 1.3 & 1.6 & 0.045 & 0.36 \\
\hline
\end{tabular}

higher order corrections). A precise estimation of these uncertainties requires a dedicated study, which is beyond the scope of this paper. Therefore here we have simply assumed that a signal can only be claimed if $R \equiv \mid N($ tot $) / N(S M)-1 \mid>5 \%$ or $R>10 \%$. For an integrated luminosity of $3000 \mathrm{fb}^{-1}$ per experiment, combining both experiments and both lepton flavours, and looking at the mass range $1500<m\left(\ell^{+} \ell^{-}\right)<3500 \mathrm{GeV}$, the reach should be $M_{c}=14 \mathrm{TeV}(10 \sigma$ statistical significance $)$ requiring $R>10 \%$ and $M_{c}=20 \mathrm{TeV}$ ( $5 \sigma$ statistical significance) requiring $R>5 \%$. As a comparison, in one year at the nominal LHC luminostiy, i.e. with $100 \mathrm{fb}^{-1}$, and considering the interval $1000<m\left(\ell^{+} \ell^{-}\right)<3500 \mathrm{GeV}$, the $5 \sigma$ reach should be $\sim 10 \mathrm{TeV}$, corresponding to a $14 \%$ deviation from the Standard Model cross-section.

In conclusion, with an integrated luminosity of $3000 \mathrm{fb}^{-1}$ per experiment, the LHC experiments should be able to detect directly KK excitations of the $\gamma$ and $Z$ gauge bosons in their leptonic decay modes for compactification scales of up to $7.7 \mathrm{TeV}$. By studying the deviation from the Standard Model expectation of the non-resonant part of the two-lepton invariant mass distribution, this reach can be extended to higher compactification scales. The sensitivity in this case is limited by the assumed uncertainty on the knowledge of the SM Drell-Yan spectrum at high mass, and is of order 15-20 TeV for a systematic uncertainty of $5-10 \%$.

\subsection{Quark substructure}

A tenfold increase in the LHC luminosity should give access to jets of up to $E_{T} \sim 4.5 \mathrm{TeV}$ (see Fig. 21), thereby extending the machine kinematic reach for QCD studies by more than $0.5-1 \mathrm{TeV}$. This improved sensitivity should have an impact also on the search for quark sub-structures. Indeed, signals for quark compositeness should reveal themselves in deviations of the high energy part of the jet cross-section from the QCD expectation. The angular distribution of di-jet pairs of large invariant mass provides an independent signature and is less sensitive to systematic effects like possible non-linearities in the calorimeter response. This method was therefore used in this study.

Figure 22 shows the expected deviations from the SM prediction for two values of the compositeness scale $\Lambda$, as a function of the variable $\chi$ defined as $\chi=(1+|\cos \theta|) /(1-|\cos \theta|)$. Here $\theta$ is the angle between a jet and the beam in the centre-of-mass frame of the di-jet system. The invariant mass of the di-jet system was required to be larger than $6 \mathrm{TeV}$. The effect of compositeness shows up as an increase in the event rate at small values of $\chi$. The compositeness scales that can be probed in this way at the LHC and SLHC are summarised in Table 16. For comparison, the potential of a $28 \mathrm{TeV}$ machine is also shown. It can be seen that a tenfold luminosity increase would have a significant impact for this physics.

As these measurements involve only the calorimeters and jets in the $\mathrm{TeV}$ range, they can be performed at the SLHC without major detector upgrades. Ability to extend the heavy-flavour tagging to the very high $E_{T}$ region could however help disentangling the flavour composition of a possible cross-section 


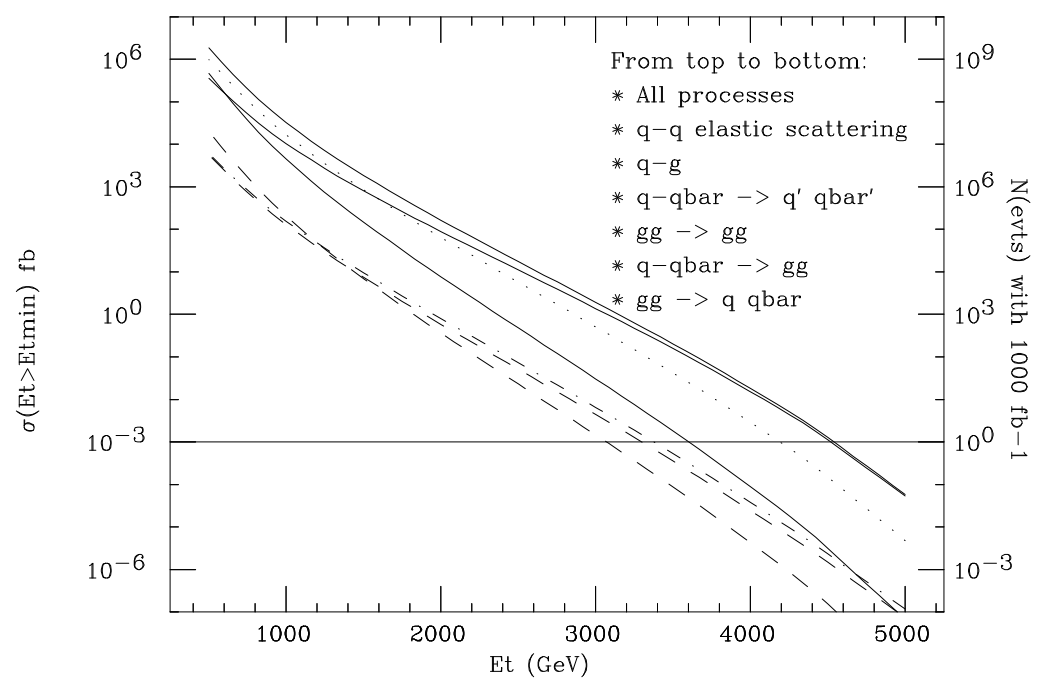

Fig. 21: Integrated production cross-section and rates for inclusive central $(|\eta|<2.5)$ jets. The different curves label the various contributions to the total the cross-section.

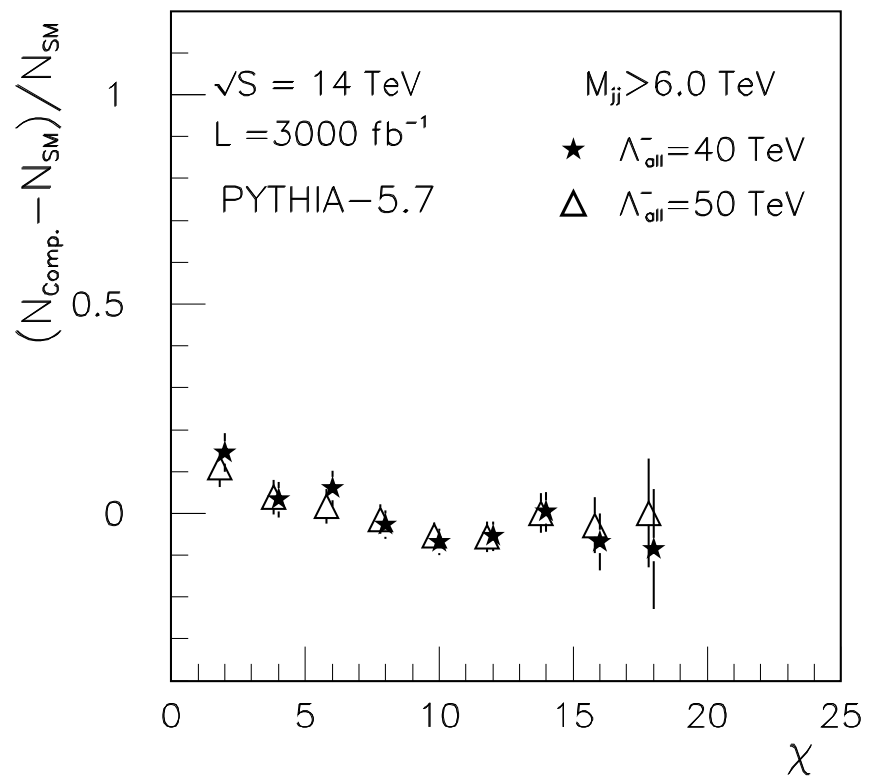

Fig.2

Fig. 22: Expected deviations from the Standard Model predictions for the angular distribution of di-jet pairs at the SLHC (ATLAS experiment, integrated luminosity of $3000 \mathrm{fb}^{-1}$ ), for two values of the compositeness scale $\Lambda$. Di-jet pairs are required to have invariant mass greater than $6 \mathrm{TeV}$.

Table 16: The 95\% C.L. lower limits that can be obtained in ATLAS on the compositeness scale $\Lambda$ by using di-jet angular distributions and for various energy/luminosity scenarios.

\begin{tabular}{|c|c|c|c|c|}
\hline Scenario & $14 \mathrm{TeV} 300 \mathrm{fb}^{-1}$ & $14 \mathrm{TeV} 3000 \mathrm{fb}^{-1}$ & $28 \mathrm{TeV} 300 \mathrm{fb}^{-1}$ & $28 \mathrm{TeV} 3000 \mathrm{fb}^{-1}$ \\
\hline$\Lambda(\mathrm{TeV})$ & 40 & 60 & 60 & 85 \\
\hline
\end{tabular}


excess. We evaluated that only a fraction smaller than $1 \%$ of the jets with $E_{T}>2 \mathrm{TeV}$ should contain bottom or charm quarks, therefore any indication of a long lifetime component in these jets beyond this level would signal New Physics.

\section{THE EXPERIMENTAL CHALLENGES AND THE DETECTOR R\&D}

The main motivation for a luminosity upgrade is to extend the physics reach of the experiments by providing more statistics. For a full exploitation of this upgrade it is imperative that the general detector performance remains at the same level as at the nominal LHC. In order to face the challenge of operation at an order of magnitude higher luminosity than foreseen in the original LHC design deliberately chosen to pose first the question of whether the currently planned detectors could survive and operate at luminosities of $10^{35} \mathrm{~cm}^{-2} \mathrm{~s}^{-1}$. If they cannot then the question is posed of possible replacements of detectors or technologies.

Development of new particle detectors takes a long time and goes through many phases starting from the idea or concept, progressing through intensive R\&D, prototyping, systems integration, installation and commissioning and finally data taking. This can be illustrated using any one of the many detector technologies in the LHC experiments. Typical time-scales stretch over more than a decade. In order to create a $R \& D$ roadmap we therefore assume that the upgraded detectors should be installed and commissioned by around 2012/2013, and try to answer the following questions:

- What R\&D is necessary?

- What priority should be assigned to the R\&D?

- When should the R\&D start, taking account of the manufacturing phase?

- What resources would be required (financial and manpower)?

- How would the R\&D interface with that carried out elsewhere?

In this Section, we shall address the above points for each sub-detector in turn, as well as for the trigger, data acquisition and electronics. While, as discussed in Section \&, several options can be envisaged for the bunch structure at high luminosiry, we limited our studies to the case of $12.5 \mathrm{~ns}$ bunch spacing.

\subsection{Inner Tracking Detector}

The performance of the tracker is characterised by momentum resolution, track reconstruction efficiency and b-tagging efficiency and purity. In order to keep the occupancy and two-track resolution at the standard LHC levels, the cell sizes have to be decreased in order to compensate for the increased track density at SLHC. Cell sizes have to be decreased by a factor 10, though simulations will be needed to optimise the granularities required at a given radius. The total cost should not much exceed that of the currently planned trackers, implying that the cost per channel has to be decreased by a factor of 10 . Cost reduction should therefore be a driving feature of any planned $R \& D$.

In what follows we assume that the tracker will be equipped with electronics that is fast enough to distinguish individual crossings. Otherwise, at $10^{35} \mathrm{~cm}^{-2} \mathrm{~s}^{-1}$, the detectors will have to deal with about 200 collisions per $25 \mathrm{~ns}$, producing about 1200 charged tracks per unit of pseudo-rapidity.

The limiting factor for the lifetime of the detectors will be radiation damage, which for cooled silicon detectors is mainly a function of the integrated luminosity. For the latter we assume $2500 \mathrm{fb}^{-1}$, which is 5 times more than the assumption used e.g. in the CMS Technical Design Report for the design luminosity of LHC. The integrated fluence and dose for the CMS Tracker are given in Table 17. Because the radiation environment is dominated by the pp secondaries, these values are of rather generic nature and not strongly CMS specific.

\footnotetext{
${ }^{3}$ For further reference the neutron flux at $10^{35} \mathrm{~cm}^{-2} \mathrm{~s}^{-1}$ and the dose for an integrated luminosity of $2500 \mathrm{fb}^{-1}$ are shown in Fig. 23.
} 


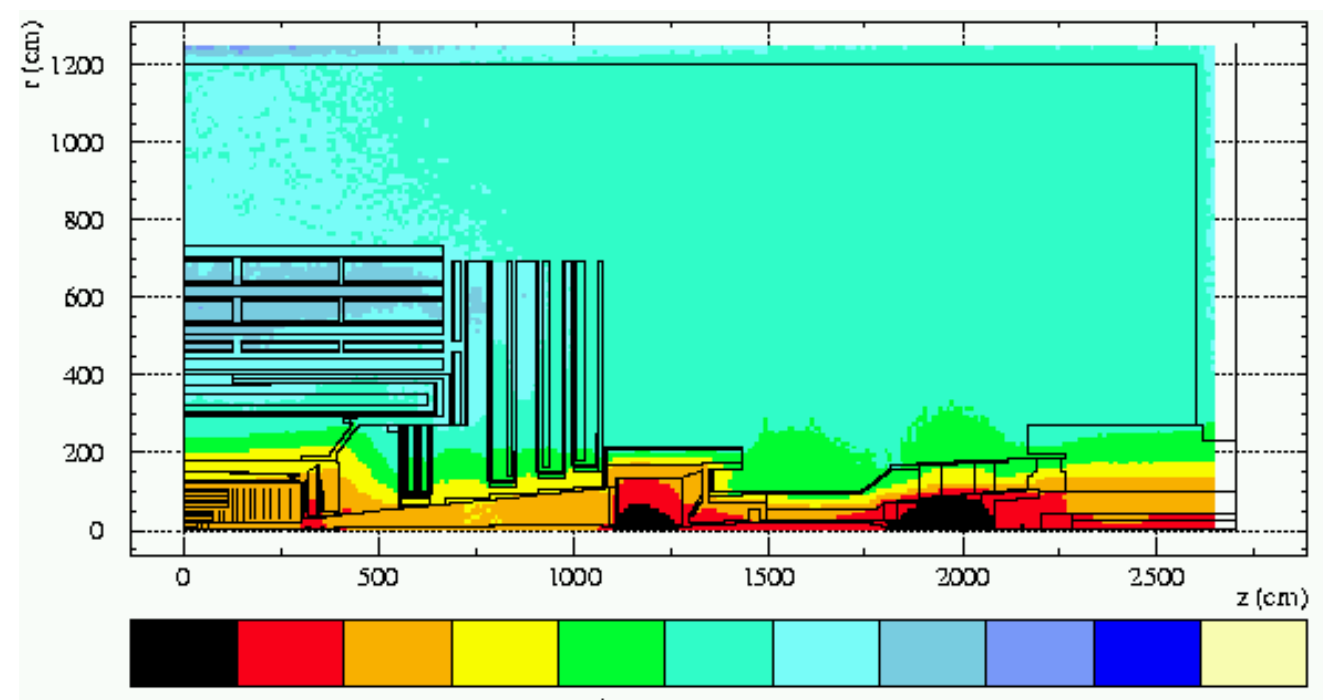

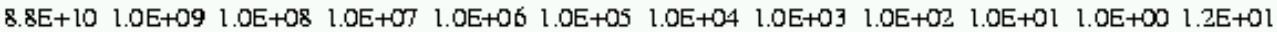

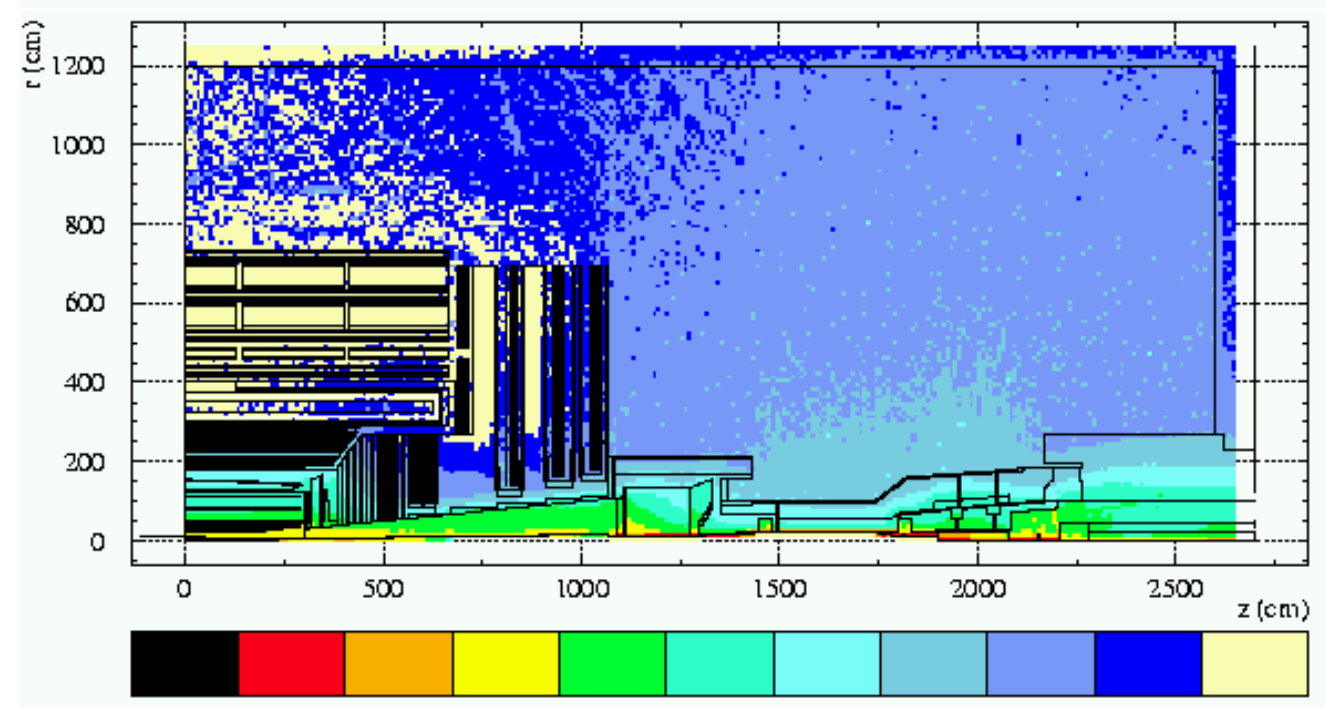

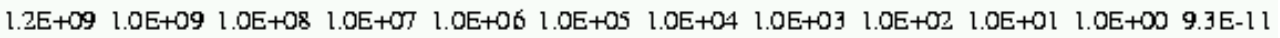

Fig. 23: Upper: the neutron flux $\left(\mathrm{cm}^{-2} \mathrm{~s}^{-1}\right)$ at an instantaneous luminosity of $10^{35} \mathrm{~cm}^{-2} \mathrm{~s}^{-1}$. Lower: dose (Gy) for an integrated luminosity of $2500 \mathrm{fb}^{-1}$

Table 17: Hadron fluence and radiation dose in different radial layers of the CMS Tracker (barrel part) for an integrated luminosity of $2500 \mathrm{fb}^{-1}$.

\begin{tabular}{|c|c|c|c|}
\hline Radius (cm) & $\begin{array}{c}\text { Fluence of fast } \\
\text { hadrons }\left(10^{14} \mathrm{~cm}^{-2}\right)\end{array}$ & Dose (kGy) & $\begin{array}{c}\text { Charged Particle } \\
\text { Flux }\left(\mathrm{cm}^{-2} \mathrm{~s}^{-1}\right)\end{array}$ \\
\hline 4 & 160 & 4200 & $5 \times 10^{8}$ \\
\hline 11 & 23 & 940 & $10^{8}$ \\
\hline 22 & 8 & 350 & $3 \times 10^{7}$ \\
\hline 75 & 1.5 & 35 & $3.5 \times 10^{6}$ \\
\hline 115 & 1 & 9.3 & $1.5 \times 10^{6}$ \\
\hline \hline
\end{tabular}


The currently planned silicon systems (designed for integrated luminosities of approximately $500 \mathrm{pb}^{-1}$ ) would probably be nearing the end of their lifetime at the start of a potential SLHC programme. They would not be able to handle the fluences at SLHC. The silicon sensors, both strip and pixel systems, would suffer substantial radiation damage, increased noise and thermal runaway as a consequence of the increased leakage currents. Since the current sensor design would not support the bias voltage required for full depletion, the sensors would operate under-depleted, decreasing the signal and increasing the noise, and hence degrading the performance.

For the electronics the situation is somewhat more favourable. The indications are that deep sub micron (DSM) electronics can probably withstand higher doses, but is not fully characterised at the doses and fluences under discussion. Mitigation of single event effects will also have to be carefully investigated. At low radius it is unlikely that the current DSM chips could survive. We also expect the opto-electronic components to be affected, and most of the materials, glues, and cooling fluids are not tested to the doses discussed above.

The innermost strip layers (at a radius of around $25 \mathrm{~cm}$ ) will have occupancies above $10 \%$. The TRT in ATLAS will face occupancy approaching $100 \%$ and cannot be used. So even if the detectors could tolerate the radiation damage, they will not be suitable for the SLHC.

We conclude that the only viable solution is to completely rebuild the Inner Detector systems of ATLAS and CMS.

Simple extrapolations based on the available experimental data show that current detector technologies, with some new developments, could work at a factor 3 larger radius, i.e. strips at $\mathrm{R}>60 \mathrm{~cm}$, pixels at $\mathrm{R}>20 \mathrm{~cm}$. This can also be seen from Table 17, where the fluences at these radii correspond to the currently foreseen fluences for strip and pixel detectors at the LHC. As a result of these rather straightforward considerations the tracker volume can be split into three radial regions:

- $\mathrm{R}>60 \mathrm{~cm}$ : where detectors can be built by further pushing existing silicon strip technology.

- $20<\mathrm{R}<60 \mathrm{~cm}$ : where further developed hybrid pixel technology can work

- $\mathrm{R}<20 \mathrm{~cm}$ : where most likely new approaches and concepts are required.

The R\&D programs should be focussed towards upgrades of ATLAS and CMS. For both experiments it is important to establish common guidelines and requirements for the relevant studies from the beginning. This implies good overall co-ordination of the R\&D and good contact between the various $\mathrm{R} \& \mathrm{D}$ groups. There are in particular two areas where common understanding and ground rules must be established with dedicated studies:

- Common software and physics studies: pattern recognition, fluences, activation, occupancy, cell size optimisation, segmentation (e.g. macro pixels, mini strips), radius of the innermost layer (driven by b-jets), material effects on the key physics channels to be studied at SLHC.

- Tracker system studies: understand the timing, synchronisation, readout/trigger rates and limitations, engineering issues (see Section 5.5).

This focus is necessary to make appropriate choices and set priorities relatively early in the R\&D phase. In the following Sections we discuss these three regions and propose and motivate various R\&D programs for suitable tracking detectors.

\section{1 .1 Tracking at a radius greater than $60 \mathrm{~cm}$.}

Silicon micro-strip detectors can be used to instrument the outer tracker $(60<\mathrm{R}<110 \mathrm{~cm})$ in a layout similar to that chosen by CMS.

Six layers of silicon micro-strip detectors with read-out pitch ranging between 80 and $160 \mu \mathrm{m}$

would be good enough to cope both with the occupancy expected for operation at $10^{35} \mathrm{~cm}^{-2} \mathrm{~s}^{-1}$ and have a point resolution needed for good transverse momentum resolution. By extrapolating estimates of 
occupancy in the CMS Si tracker at nominal LHC the global occupancy is expected to vary from less than $1 \%$ in the outer layers to a maximum of a few $\%$. In this region a $50 \%$ increase in number of channels would probably be needed compared to the current CMS strip tracker.

The fluence of fast hadrons in this region for an integrated luminosity of $2500 \mathrm{fb}^{-1}$ is estimated to be between 1 and $3 \times 10^{14} \mathrm{~cm}^{-2}$. Extensive studies have been done in preparation of the current generation of LHC inner trackers to validate the use of silicon detectors up to integrated fluences of $1.5 \times$ $10^{14} \mathrm{~cm}^{-2}$ [44, 45]. In particular it has been demonstrated that the current high breakdown technology $\left(V_{\text {break }}>500 \mathrm{~V}\right)$ guarantees safe operation of these devices for the entire LHC lifetime.

For SLHC many of the characteristics of what are considered to be standard "radiation resistant" silicon micro-strip detectors will be maintained: $\mathrm{p}^{+} \mathrm{n}$ technology, integrated AC coupling, poly-silicon bias circuit, $\langle 100\rangle$ crystal orientation, standard $0.20-0.25 \mathrm{w} / \mathrm{p}$ ratio and metal overhang on the strips. A careful study will be needed on the charge collection efficiency in heavily irradiated devices read-out by very fast shaping time electronics. A focused $R \& D$ program will be needed to optimise the Si-strip detector characteristics, performance and cost.

A factor of 2 higher fluence in SLHC would require further improvement in breakdown voltage in industrially produced devices though use of lower resistivity silicon and thinner devices could lower the bias voltage at full depletion. The choice of wafer resistivity and thickness will have an impact on system aspects (detector module layout and strip length, read-out granularity, noise performance of the front-end electronics, cooling needs etc) and on the total cost (number of channels, number of detectors, technology and cost of processing).

The microelectronics industry is rapidly migrating to 8" (and then to 12") processing lines. It is likely that the currently used 6" lines will not be available in 6-8 years from now. As a consequence a part of the R\&D program should be devoted to the exploration of the feasibility of processing of detector grade Si wafers with a larger diameter, and to transfer the existing detector processing technology to the new lines.

\section{1 .2 Tracking between a radius of $20 \mathrm{~cm}$ and $60 \mathrm{~cm}$.}

Today's pixel technology is expected to work at radii above $20 \mathrm{~cm}$. Questions related to the infrastructure, services, power and cost are likely to be decisive in determining the minimum radius at which the pixels can be deployed at the SLHC.

It is possible to marry the current pixel architecture (both sensor and electronics) for cell-sizes that are ten times bigger than the current pixels but ten times smaller than the size of the current microstrips in this region. Hence a critical goal of the necessary R\&D would be to achieve a cost/pixel that is between 10 and 100 times smaller than the current cost/microstrip. Such devices can be labelled 'macro-pixel' devices. Many issues such as routing will need to be addressed. Such devices could already figure in the upgrades of innermost silicon strip detectors of current LHC trackers.

Defect engineered silicon (section 5.1.4.a) is already used in the current LHC trackers, in particular to reduce damage due to charged hadron irradiation at low radius. Defect engineered silicon has the potential of reducing the radius at which current technologies can be used at SLHC.

\subsubsection{Tracking at a radius smaller than $20 \mathrm{~cm}$.}

At the LHC design luminosity the innermost pixel detectors are expected to be placed at a radius of $7 \mathrm{~cm}$ from the beamline. The occupancy at $7 \mathrm{~cm}$ is estimated to be $\simeq 3 \times 10^{-4}$. At SLHC, in order to preserve the occupancy at a tolerable level at such radii, the pixels area should be decreased by at least a factor of 5. The b-tagging performance should then not be degraded.

At SLHC, and at a radius of $\simeq 7 \mathrm{~cm}$, the dose and fluence of hadrons is expected to be $100 \mathrm{kGy}$ and

$5 \times 10^{15}$ hadron $/ \mathrm{cm}^{2}$ respectively. Hence this is an extremely harsh region. Short of changing the hybrid 
pixel detectors annually, or perhaps even more frequently, there are currently few solid and demonstrated possibilities. Hence fundamental research rather than only development is needed. New concepts, geometries and materials are probably needed to attain the required speed and radiation hardness.

The pixel systems in the current trackers will possibly be changed during the LHC period and some of the R\&D mentioned above will also be very relevant for these upgrades.

\subsubsection{Subjects for $R \& D$}

\section{a) Use of defect-engineered silicon}

The term "defect-engineering" stands for the deliberate incorporation of impurities or defects into the silicon bulk material before or during the processing of the detector. The aim is to suppress the formation of microscopic defects that have a detrimental effect on the macroscopic detector parameters during or after irradiation. In this sense defect engineering tries to cope with the problem of radiation damage at its root.

One example of a defect-engineered material is the "Diffusion Oxygenated Float Zone" (DOFZ) silicon developed by the ROSE (CERN RD48) collaboration [46]. It was shown that this oxygen-enriched material exhibits an improved radiation tolerance with respect to charged hadron irradiation. The increase of depletion voltage after type inversion is reduced by a factor of three and the so-called "reverse annealing" saturates at fluences above about $2 \times 10^{14} \mathrm{~cm}^{-2}(24 \mathrm{GeV} / \mathrm{c}$ protons $)$. Furthermore, the reverse annealing is slowed down allowing for longer warm up (maintenance) periods. These properties and the relatively simple and cheap implementation into detector processing make DOFZ an ideal material for the detectors located closest to the interaction points. After thorough testing the ATLAS collaboration is now using it for the pixel layers. It is anticipated that the CMS pixel collaboration will follow the same path.

It is expected that further optimisation studies of the oxygenation process will lead to better results with respect to radiation tolerance. Furthermore it is worth exploring other promising possibilities for defect-engineering [47].

\section{b) 3D detectors and new biasing schemes}

The main characteristic of '3D' detector concept is to place the electrodes $(\mathrm{p}, \mathrm{n})$ throughout the bulk in the form of narrow columns instead of being deposited parallel to the detector surface. In a conventional silicon sensor the depletion and charge collection across the full wafer thickness (usually $300 \mu \mathrm{m}$ ) requires high voltages and becomes incomplete after intense irradiation. The main advantage of the 3D approach is the short distance between the collecting electrodes. This allows very low depletion bias voltage $(\simeq 10 \mathrm{~V})$ as well as very fast collection times and low noise. The principle has been successfully proven on small prototypes operated after irradiation with protons up to $2 \times 10^{15} \mathrm{n}_{1 \mathrm{MeV} \text { eq }} / \mathrm{cm}^{2}[48]$. Other approaches are also under study [49].

The $3 \mathrm{D}$ concept is a very new approach. Considering that above $10^{15}$ particle $/ \mathrm{cm}^{2}$ only electrons contribute to the generated signal, due to the rapid trapping of the holes and the reduced effective drift length of the electrons, '3D' concepts potentially are probably 'rad-hard' up to fluences of $5-10 \times$ $10^{15}$ particles $/ \mathrm{cm}^{2}$ if collection is performed at the $\mathrm{n}^{+}$-electrode. Moreover, if '3D' concept would be combined with defect engineered material and operated in forward bias at cryogenic temperatures, radiation tolerance might be achieved for even higher particle fluences. Size and uniformity of the sensors are important issues to be addressed together with optimisation of the manufacturing techniques.

\section{c) New sensor materials}

CVD diamond is almost an ideal material for radiation hard charged particle detectors. Its outstanding radiation tolerance, fast charge collection, low dielectric constant, and low leakage current make it a good candidate for high luminosity colliders.

The RD42 collaboration has demonstrated that small area strip and pixel detectors based on di- 
amonds can be fabricated; they can collect signals corresponding to about $8,000 \mathrm{e}^{-}$and work up to a proton fluence of $5 \times 10^{15} \mathrm{p} / \mathrm{cm}^{2}$ [50].

A complete qualification of the technology would require a strong partnership with the producers of the raw material to increase the collected charge and to perform basic research on defects and impurities, both before and after irradiation. In terms of processing techniques an optimization of the electrical contacts is needed. Further studies would be needed on the characterization of complete detector modules.

\section{d) Cryogenic Silicon Tracker development}

The main motivations for silicon detectors operated at $130 \mathrm{~K}$ are:

- a factor of 10 increase in radiation hardness due to the 'Lazarus' effect [51];

- a factor 5 higher mobility for both carriers which leads to very fast sensors;

- negligible bulk and surface current generation rate at high voltage, even after substantial radiation damage.

In addition the factor 3 increase in the thermal conductivity of silicon between $130 \mathrm{~K}$ and $300 \mathrm{~K}$ would facilitate significantly the engineering design of the detector modules as far as evacuation of heat is concerned.

Results of the RD39 collaboration show that prototype modules can be fabricated with simplified techniques and successfully operated at very high SPS lead ion fluences of $(5 \pm 2) \times 10^{14}$ ions $/ \mathrm{cm}^{2}$ yielding almost $100 \mathrm{Grad}$ energy deposit [52]. It was also shown that the development of fast low-noise cryogenic read-out electronics is feasible, and that the basic engineering issues of operating complex systems of low temperature detectors can probably be addressed.

The basic advantage of this approach is that, once proven to be feasible and reliable on the large scale required for the SLHC trackers, it could be used for all the three radial regions. The need for further replacement will then be mainly motivated by the limits of radiation resistance of the DSM electronics.

From the point of view of the engineering design, it would be attractive to close the ends of the cavity that would house the entire tracker in cryogenic vacuum. Microtubes integrated in the module design would evacuate the heat produced locally by the electronics. The clean vacuum would help in avoiding contamination of the detector components, and a very thin-walled beam pipe would then be feasible.

While the cryogenic micro-strip sensors and modules are already being developed with promising initial results, there is much work still to be done in optimisation and testing of the pixel devices and DSM front-end electronics for low-temperature operation. At present the current-injected or forwardbiased cryogenic sensors can be operated up to the fluence of $2 \times 10^{15} \mathrm{~cm}^{-2}$ in the strip segmentation, and perhaps 5 times higher in the pixel devices. These results were obtained, however, using simplified devices; it is clear that further R\&D is required for large area detectors to be produced with high yield and then assembled in an automated manner. The device structures and current injection schemes should be further studied and optimised. Low-temperature studies of the bulk silicon under high damage should also be pursued, with view on possible optimisation of the defect neutralization and charge carrier trapping.

The engineering studies of the cryogenic tracker would also be a critical issue for the R\&D.

\section{e) Monolithic Pixel Detectors}

In a monolithic pixel detector a two-dimensional array of detecting diodes and the associated miniaturized readout electronics are integrated on the same silicon substrate. Compared to the hybrid approach, in which the arrays of sensing elements and readout cells are manufactured on different wafers, which are then bump-bonded together, the monolithic approach presents clear advantages in terms of detector assembling and handling.

Another important advantage of the monolithic technology is the reduction of the amount of matter 
Table 18: The neutron fluence and radiation dose at shower maximum at different pseudorapidities for an integrated luminosity of $2500 \mathrm{fb}^{-1}$.

\begin{tabular}{|c|c|c|c|}
\hline $\begin{array}{c}\text { Pseudorapidity } \\
\eta\end{array}$ & $\begin{array}{c}\text { ECAL Dose } \\
(\mathrm{kGy})\end{array}$ & $\begin{array}{c}\text { HCAL Dose } \\
(\mathrm{kGy})\end{array}$ & $\begin{array}{c}\text { ECAL Dose Rate } \\
(\mathrm{Gy} / \mathrm{h})\end{array}$ \\
\hline $0-1.5$ & 15 & 1 & 2.5 \\
\hline 2.0 & 100 & 20 & 14 \\
\hline 2.9 & 1000 & 200 & 140 \\
\hline 3.5 & - & 500 & - \\
\hline 5 & - & 5000 & - \\
\hline \hline
\end{tabular}

to be traversed by the particles. Moreover, the very small capacitance (down to few fF) presented by the detecting element at the input of the close-by front-end transistor result in reduced noise.

Monolithic pixel detectors will only be attractive if standard technologies can be used to keep costs affordable. A key issue is to understand whether fast switching front-end electronics needed for the SLHC can be integrated on the same piece of silicon with the detecting element. Recent tests of such sensors are promising though it is not clear whether the required level of radiation hardness will be achievable.

\subsubsection{Engineering aspects}

For the support structures a highly modular approach based on carbon fibre composite elements seems to be appropriate to cope with the increased radiation levels of SLHC. Moreover a large part of the existing outer supporting structures could probably be copied.

An increased number of channels and higher radiation levels would increase cooling needs. Since the requirements will be driven by the inner parts of the tracker, and a unique operating temperature for the entire tracking volume is advisable, the most likely operating scenario foresees an overall temperature $\mathrm{T} \simeq-15 /-20^{0} \mathrm{C}$. We presume that the currently foreseen cooling techniques could be used.

The power requirements for the front-end electronics should be defined early to enable implementation of radiation-hard local voltage regulators. Their use is mandatory in order to reduce the mass of power cables which otherwise would be one of the dominant contributors to the material budget.

Extensive use of high density interconnections, low mass hybrids and flexible circuitry will be necessary to reduce the amount of material for the read-out and ancillary electronics.

It will be important to maintain some level of accessibility for maintenance of critical detector components.

\subsubsection{Electronics}

\section{See Section 5.5}

\subsection{Calorimetry}

The chosen calorimeter technologies of ATLAS and CMS [53, 54, 55, 56] are designed to withstand an integrated luminosity in excess of $500 \mathrm{pb}^{-1}$. ATLAS has chosen liquid argon sampling calorimetry for the electromagnetic, the endcap hadronic and the forward calorimeters. The barrel hadronic calorimeter comprises iron/plastic scintillator sandwich with wavelength shifting fibres. CMS is using scintillating crystals for the electromagnetic, and brass/plastic scintillator sandwich with wavelength shifting fibres for the hadronic calorimeter. The forward calorimeter uses quartz fibres embedded in grooves in iron plates. The neutron fluence and radiation dose at shower maximum and at different pseudorapidities for an integrated dose of $2500 \mathrm{fb}^{-1}$ are given in Table 18. 
Table 19: Comparison of the critical density with the energy density for ATLAS liquid argon calorimeters

\begin{tabular}{|c|c|c|c|}
\hline & Critical density & ATLAS $10^{34}$ & ATLAS $10^{35}$ \\
\hline Barrel EM, $\eta=0$ & $5 \times 10^{6}$ & $0.5 \times 10^{5}$ & $5 \times 10^{5}$ \\
\hline Barrel EM, $\eta=1.3$ & $4 \times 10^{6}$ & $1.2 \times 10^{5}$ & $1.2 \times 10^{6}$ \\
\hline End-cap EM $\eta=1.4$ & $3 \times 10^{6}$ & $1.3 \times 10^{5}$ & $1.3 \times 10^{6}$ \\
\hline End-cap EM $\eta=3.2$ & $5 \times 10^{6}$ & $2.5 \times 10^{6}$ & $25 \times 10^{6}$ \\
\hline FCAL $\eta=3.2$ & $1500 \times 10^{6}$ & $2.5 \times 10^{6}$ & $25 \times 10^{6}$ \\
\hline FCAL $\eta=4.5$ & & $130 \times 10^{6}$ & $1300 \times 10^{6}$ \\
\hline \hline
\end{tabular}

Below we consider the possible limitations of these techniques and calorimeters for a 10 -fold increase in instantaneous luminosity, and a 5-fold increase in integrated luminosity.

\subsubsection{Liquid Argon Calorimeter}

The ATLAS Calorimeter was optimised for the nominal LHC luminosity of $10^{34} \mathrm{~cm}^{-2} \mathrm{~s}^{-1}$ and a centre of mass energy of $14 \mathrm{TeV}$. A factor of 10 increase in this luminosity would raise concerns that are considered below.

\section{a) Space charge effects.}

During steady operation of the calorimeter, an equilibrium is reached between ion creation by the passage of charged particles and ion collection by the electric field in the gaps. When the positive ion space density integrated over the gap becomes comparable to the charge density on the electrodes due to the HV polarisation, the field is distorted, and a distortion of the response may occur. Fortunately, no practical change in the response occurs until a region near the anode with zero field appears. The onset of such a regime goes like $V^{2} / d^{4} \mu$, where $\mathrm{V}$ is the operating voltage, $\mathrm{d}$ the gap and $\mu$ the $\mathrm{Ar}^{+}$mobility. This last quantity is not very well measured experimentally, and so far we have had more confidence in checking directly the onset of some saturation. Measurements in test beam using prototypes of the ATLAS EM calorimeter [53] show that losses at the level of $1 \%$ occur for an energy flux of about $5 \times 10^{6} \mathrm{GeV}$ $\mathrm{cm}^{-2} \mathrm{~s}^{-1}$

Table 19 compares the "critical density" with the energy density in various parts of ATLAS liquid Argon calorimeter for two values of the luminosity. The critical density in the various areas is scaled from the measured number, taking into account the actual geometry and the sampling fraction, as well as the shower extension in length. A major step occurs for the FCAL with gaps as narrow as $0.25 \mathrm{~mm}$ in a dense tungsten matrix

The numbers in the Table indicate a comfortable margin in the barrel, while the inner parts of the EM endcap, and of the FCAL may be affected. Ways to stay away from limits set by the space charge effects have to be investigated. These could involve different liquids (perhaps liquid krypton),or a cold dense gas under pressure (compatible with what the cryostats can withstand).

\section{b) Voltage drop in the HV distribution}

The current induced by the drift of electrons and ions in the gap, circulates in the HV polarisation chain, which incorporates resistors to isolate from each other channels hooked to the same HV supply. The value of these resistances should not be too low in order to avoid cross-talk, and not too high such that the induced drop would require a rate dependent correction.

The value of the resistances is about 10 times larger at LAr temperature than at room temperature. This factor $\simeq 10$ has large fluctuations from pad to pad (rms/average $\simeq 0.3$ ) which precludes using measurements made at room temperature to correct for the drop.

The expected voltage drops are given in Table 20. As for the ion build-up there is a comfortable margin for the barrel. The "small wheel" of the EM endcap, because of its smaller number of electrodes 
Table 20: The voltage drops expected in ATLAS liquid argon calorimeters

\begin{tabular}{|c|c|c|c|c|}
\hline & Resistance/0.05 & Current at $10^{34}$ & $\begin{array}{c}\text { Voltage drop } \\
10^{34}\end{array}$ & $\begin{array}{c}\text { Voltage drop } \\
10^{35}\end{array}$ \\
\hline Barrel EM, $\eta=0$ & $\sim 1 \mathrm{Mohm}$ & $80 \mathrm{nA}$ & $0.08 \mathrm{~V}$ & \\
\hline Barrel EM, $\eta=1.3$ & & $200 \mathrm{nA}$ & $0.2 \mathrm{~V}$ & $2 \mathrm{~V}$ \\
\hline End-cap EM, $\eta=2.4$ & & $400 \mathrm{nA}$ & $0.4 \mathrm{~V}$ & $4 \mathrm{~V}$ \\
\hline End-cap EM, $\eta=2.5$ & & $4000 \mathrm{nA}$ & $4.0 \mathrm{~V}$ & $40 \mathrm{~V}$ \\
\hline End-cap EM, $\eta=3.2$ & & $8000 \mathrm{nA}$ & $8.0 \mathrm{~V}$ & $80 \mathrm{~V}$ \\
\hline \hline
\end{tabular}

(256 against 768) sees a large current per sector. Significant effects are expected in this area, which would not allow precision measurements.

In order to get rid of the limitation induced by this effect, a different liquid should be explored, with less charge deposited per $\mathrm{GeV}$ or one that is at higher temperature. A cold dense gas should also be evaluated in this respect.

\section{c) Activation}

While this affects the "logistics" and not the detector response itself, activation may become a very serious limitation for the practical use of the detector. In particular for ATLAS one would have to reconsider the level of $\mathrm{Ar}^{40}$ that can be released into the atmosphere in case of evacuation of the liquid due to a fault in the cryogenic system.

\section{d) Radiation damage to the detector}

There is a comfortable margin in this respect. The only measurable effect found so far is a small increase of the polarisation resistances, that are silk screened on electrodes (see section b), under neutron irradiation.

\section{e) Radiation damage to the electronics}

Apart from the HEC cold preamplifiers, all active circuits are in warm environment, and therefore accessible and replaceable if needed. By design all ASICs (including the HEC GaAs preamplifiers) were manufactured in a technology offering a large safety margin w.r.t. the expected radiation level at the nominal high luminosity of LHC.

Nevertheless a dedicated analysis would be needed to evaluate the potential problems for 10 times more radiation. Particularly critical may be the case of the few COTS (commercial components) used in the front-end crates.

\section{f) Sequencing of the readout}

The effect of shorter bunch spacing, such as $12.5 \mathrm{~ns}$, has to be evaluated though the current scheme may well be adequate. In "standard" ATLAS conditions calorimeter pulses are sampled every $25 \mathrm{~ns}$, and the energy and time are calculated using "optimal filtering" with data from 5 consecutive samples. Timing is arranged such that the third sample falls at the maximum of the pulse.

In the test beam, which is asynchronous, all events in a window of $\pm 12.5 \mathrm{~ns}$ around the optimum timing are used, and give results indistinguishable from those sampled at the peak. In the case of 12.5 ns bunch spacing, one could therefore continue to clock the readout at $40 \mathrm{MHz}$, requiring only that the LVL1 identifies whether the triggered event is synchronous with this clock or advanced by $12.5 \mathrm{~ns}$.

\section{g) Formation of LVL1 signals}

The bandwidth of the present LVL1 authorises "BCID” for all signals above a given threshold. To obtain a similar "BCID" at $80 \mathrm{MHz}$ is likely to require an upgrading of this system. Since everything is accessible, such a change would cost money but should not be an a priori show-stopper 


\section{h) Level of pileup}

With no change to the circuits nor to the signal treatment, a factor of 10 in luminosity translates into a factor of 3 in pile-up, while electronics noise in unchanged. An optimal filtering could allow to "speedup" the equivalent response, for a better balance between electronics and pile-up noise, leading to a smaller degradation.

The use of a different liquid, or cold dense gas, would indeed require a full re-evaluation of the signal to noise ratio, and in general of the calorimeter performance.

In conclusion, an increase of luminosity would certainly create space charge and voltage drop problems in the large $\eta$ region of the Endcap electromagnetic calorimeter $(\eta>2.5)$ and the forward calorimeter (FCAL). R\&D will be needed to understand better these effects, and to investigate the use of other liquids or of a cold dense gas.

\subsubsection{CMS Crystal ECAL}

\section{a) Crystals}

In case of an order of magnitude luminosity increase, the dose rate in the Barrel (at shower max) will increase from $0.15 \mathrm{~Gy} / \mathrm{h}$ to $1.5 \mathrm{~Gy} / \mathrm{h}$, which corresponds to the current nominal situation at $\eta=2.4$. There should therefore be few problems. For the endcaps, the dose rate reaches $30 \mathrm{~Gy} / \mathrm{h}$ at $\eta=2.6$ and $75 \mathrm{~Gy} / \mathrm{h}$ at $\eta=3$. This is close to the "saturation" irradiation conditions actually used at the Geneva Hopital Cantonal $(250 \mathrm{~Gy} / \mathrm{h})$. It is known that the light attenuation in this condition is $>1$ meter and therefore the light loss is less than $25 \%$.

However, for a complete understanding, a programme of irradiations under these conditions should be performed including

- long-term irradiation (days)

- irradiations with high fluxes of hadrons, for comparison with gammas.

- calibration studies

\section{b) Photosensors}

The leakage current in the APDs used in the Barrel will increase by approx $20 \mu \mathrm{A}$ per year at SLHC. This should translate into a large increase in electronics noise, reaching $100 \mathrm{MeV}$ per crystal after a few years. Improved recovery mechanisms (e.g. higher temperature during shut down) could be investigated.

For Endcap VPTs the glass window has to be tested at the expected very high radiation doses. The behaviour of the tube with a strong steady current should also be studied.

\section{c) On-detector Electronics}

\section{Radiation hardness.}

The electronics is built using radiation-hard processes that are qualified for the nominal situation in the endcap (5Mrad, $2 \times 10^{14} \mathrm{n} / \mathrm{cm}^{2}$ for an integrated luminosity of $500 \mathrm{fb}^{-1}$ ). One can therefore conclude that the electronics in the Barrel will survive an order of magnitude luminosity increase. The situation for the endcaps is much more critical. The actual electronics has already been recessed from the beam axis to limit the maximal fluence to $2 \times 10^{14} \mathrm{n} / \mathrm{cm}^{2}$. An increase by a factor 10 requires either a move from the actual position to the periphery -which is probably impossible - or a replacement after a vigorous $\mathrm{R} \& \mathrm{D}$ to find more radiation hard technologies. This affects all the components of the Front-End cards (preamplifier, ADC, optolinks)

Faster bunch crossing.

Running the actual electronics at $80 \mathrm{MHz}$ is impossible. As in the case of the ATLAS liquid Argon calorimeter, the first thoughts are that one could cope with a doubling of the frequency (12.5 ns bunch crossing) by still sampling at the original $40 \mathrm{MHz}$ frequency. The excellent time resolution obtained with the multi-sample electronics would allow a corect assignment of the bunch crossing The LV1 trigger primitives are created by filters using 5 consecutive samples. The consequences on the actual CMS 
Trigger system have to be studied.

\section{d) Pileup}

Pileup effects have to be assessed, both from the physics point of view and also for the electronics (for example the variation of the base line for the Floating Point Preamplifier). The last point may be an issue in the high $\eta$ range.

\section{e) Activation}

One of the biggest worries is the activation level that would be reached in the endcaps,; certainly several $\mathrm{mSv} / \mathrm{h}$ after a run at a luminosity of $10^{35} \mathrm{~cm}^{-2} \mathrm{~s}^{-1}$. This means that interventions will be very difficult (the integrated allowed yearly dose being reached in a few hours). One should not envisage regular replacements of hardware in these conditions.

The results of the radiation hardness tests suggested above for VPTs and for electronics are therefore crucial. One should note that already the replacement campaign for the endcaps electronics before the upgraded Luminosity period will require careful planning.

In summary the CMS ECAL Barrel could probably be used with an increase of luminosity, even if the performance may be somewhat degraded due to an increase in noise and pileup. The situation for the Endcap is more difficult to assess: $R \& D$ is required to verify the behaviour of the crystals and photosensors under high dose rates. The Front End electronics will have to be replaced in difficult activation conditions.

\subsubsection{Plastic Scintillator Based Hadron Calorimeters}

CMS employs a brass/plastic scintillator sampling hadronic calorimeter up to $|\eta|<3$. ATLAS uses a similar technique in the barrel region. The plastic scintillator used in these calorimeters loses half of its light output after a dose of about $50 \mathrm{kGy}$. Hence, from Table 18, it can be seen that the ATLAS and CMS hadron calorimeters in the barrel region $(|\eta|<1.5)$ should not need changing.

The situation is more difficult for the CMS endcap hadron calorimeter. The deleterious effects of radiation can be substantially mitigated by individully reading out the scintillator layers in the first 3-4 interaction lengths. The signals from these layers can then be weighted to compensate for the loss of light. Periodic replacement of the scintillator, albeit difficult, could also be envisaged. A programme of $\mathrm{R} \& \mathrm{D}$ should be undertaken to search for an alternative active medium and for a more radiation tolerant scintillator.

\subsubsection{CMS Very Forward Calorimeter}

The radiation dose in the forward region $(3<|\eta|<5)$ changes rapidly. The CMS iron/quartz fibre calorimeter uses plastic-clad quartz fibres. Quartz -clad quartz fibres are more radiation resistant but are much more expensive. Replacing plastic-clad fibres by quartz-clad fibres, will allow the use of the same technique up to integrated luminosity of $2500 \mathrm{fb}^{-1}$.

Novel technologies that can operate at ultra high radiation levels should be searched and developed. A possibility is to detect the Cerenkov light emitted in pressurised gas contained in $2 \mathrm{~mm}$ diameter steel tubes whose reflectivity on the inner surface at grazing incidence is very high. The idea has been tested in CERN-H4 beam [57]

\subsection{Muon Systems}

\subsubsection{Intensity considerations}

\section{Background radiation}

The ATLAS and CMS muon systems [58, 59] have been designed according to conservative assumptions in the background rates (factor 3 to 5 safety margin above estimates from simulations). The real safety 
margin can only be established from measurements once LHC operates. It is possible that in some regions measured rates will be substantially higher than current expectations and this information, available only five years hence, will influence the modifications necessary for SLHC.

The particle fluence scales with luminosity and is an even stronger function of $\eta$ and $\mathrm{r}$ (factor of 100 variation). It is predominantly composed of low energy $(<100 \mathrm{MeV})$ neutrons, high energy neutrons and photons (with typical energy $<10 \mathrm{MeV}$ ). At SLHC, the fluence of each species ranges up to some $10^{5} \mathrm{~cm}^{-2} s^{-1}$ in the highest $\eta$ region. The detection efficiency for these particles is in the range of 0.1-1 $\%$. They dominate the observed hit rate at low $\eta(\leq 2.2)$. The rate for charged particles (hadrons, muons, isolated electrons) is typically much lower than the rate of detected neutral ones, except in the high $\eta$ region (2.2-2.7), where it progressively becomes dominant.

When considering technologies for use at SLHC, it is worth pointing out that the strong geometric dependence implies that detector types which function at high- $\eta$ in LHC will certainly work quite adequately at low- $\eta$ in SLHC.

\section{Shielding and muon tracking in the high- $\eta$ region}

The simplest viable modification for SLHC is to increase shielding around the beam-pipe at high- $\eta$, which reduces the overall background rate and is particularly effective at low- $\eta$, where neutrals dominate the observed hit-rate in the muon detectors. The penalty is a cut in the high- $\eta$ acceptance. However, the rate from charged particle background (20-40\% muons), effectively irreducible by shielding, may in any case limit the deployment of detectors in the forward regions, and force a reduction in the acceptance at SLHC.

Figure 24 compares the contours of fluence in the low radius, high- $\eta$ region of the CMS detector, for a high- $\eta$ cut-off of 2.4 (present LHC shielding) and 2.0 (possible shielding for SLHC). The maximum rates to which the cathode strip chambers are exposed are similar in the two cases, thus a re-build of the low radius muon layers to match the new geometry could be done using the existing technologies. The CMS resistive plate chambers used in triggering are already limited to the $|\eta|<2.1$ region. Depending on the exact configuration of machine elements chosen for the SLHC low- $\eta$ insertions (see below), re-design of the shielding around the TAS collimator, and around the beam-pipe within the forward muon system might also imply significant engineering re-design of the entire forward regions of the experiments, for instance the forward toroids of ATLAS or the forward calorimetry system of CMS. All this adds to the uncertainty in the rate estimates, and it is not inconceivable that in the worst case the first forward muon stations might need to be replaced by a different design concept. However, the aim would be to use developments of technologies already proven at LHC in a worse environment, eg those that were candidates for central tracking.

Tracking in the low- $\eta$ region $(\eta<2)$.

Assuming that an effective forward shielding design can be maintained, the current muon detectors in the central region $\eta<2$, will mostly perform well enough. However, the insensitivity to ageing at integrated dose levels higher than considered so far may need to be confirmed in some cases. The expedient of substituting high- $\eta$ technologies in the most vulnerable low- $\eta$ regions, could lead, in the CMS case, to replacement of drift tube chambers by endcap-type cathode strip chambers in the first and fourth barrel stations. However, the detector performance may be otherwise affected by the increased background e.g., the resolution of the ATLAS MDTs may be limited by space charge effects (which may already reduce performance at the highest $\eta$ at LHC). Experience from LHC operation is necessary to make an informed judgement.

\subsubsection{Trigger}

The design of the trigger will need a significant upgrade to cope with the increased intensity. In particular, rejection ability considerations will force the trigger to be driven at the increased bunch-crossing rate of $80 \mathrm{MHz}$. Different considerations apply to the different detector technologies: 

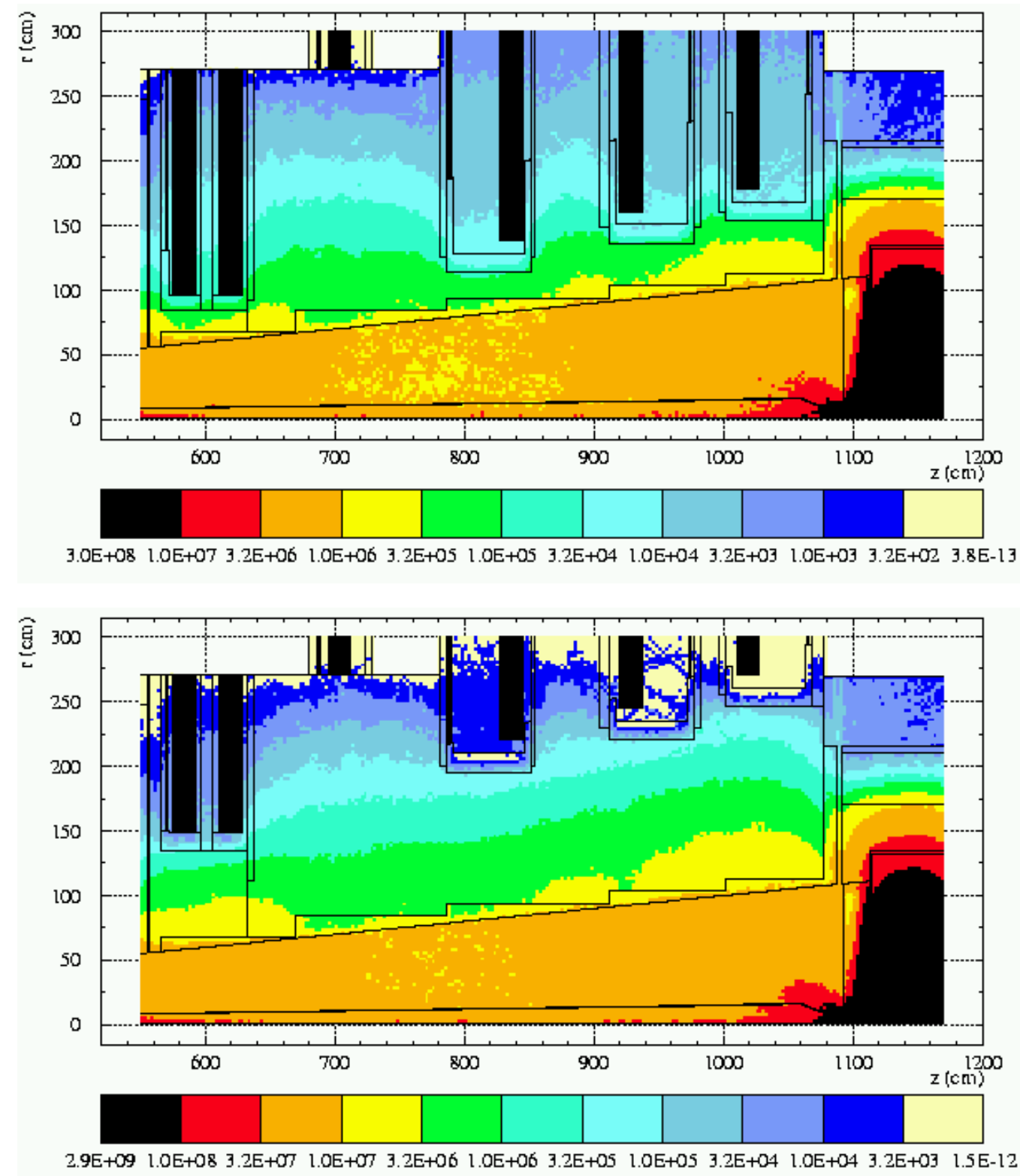

Fig. 24: Upper: the neutron fluxes $\left(\mathrm{cm}^{-2} \mathrm{~s}^{-1}\right)$ in the low radius, high $\eta$-region of the CMS endcap muon detector for $\eta<2.4$ and present LHC shielding $10^{34} \mathrm{~cm}^{-2} \mathrm{~s}^{-1}$. Lower: same, for for $\eta<2$ and possible shielding for SLHC $10^{35} \mathrm{~cm}^{-2} \mathrm{~s}^{-1}$. 
- For intrinsically fast detectors (i.e. with signal generated with time jitter $<12.5 \mathrm{~ns}$ ), where the trigger decision is taken asynchronously (or over-sampling above $40 \mathrm{MHz}$ ) (e.g.: the ATLAS RPCs), part of the existing trigger might be usable at SLHC.

- For fast detectors with trigger logic effectively driven by the LHC $40 \mathrm{MHz}$ bunch-crossing rate (e.g. ATLAS TGCs, CMS Drift Tubes); the trigger logic would need to be redesigned to operate at $80 \mathrm{MHz}$.

- For slow detectors, with signal time jitter comparable or above 12.5 ns (e.g. the ATLAS TGCs in the largest $\eta$ region: for tracks at large incidence angle); a different detector technology, or at minimum a more sophisticated electronics, might have to be considered in order to operate in these regions.

\subsubsection{Read out}

The detector read out would have to cope with increased bandwidth, due to higher background rates, and possibly to higher trigger rate. The actual read out speed may or may not need to be upgraded (e.g., the drift tubes could presumably be read out with the same speed and memory depth).

There may be sufficient impact on power dissipation in certain detectors to require an upgraded cooling system. Even if most existing detector elements are usable at luminosities around $10^{35} \mathrm{~cm}^{-2} \mathrm{~s}^{-1}$, the time taken to replace on-board electronics and cooling may be a major contribution to the shutdown length needed to re-configure experiments for SLHC.

\subsubsection{Beam optics and radio-activation}

The increase in luminosity would be achieved in part by doubling the bunch-crossing rate, in part by an increase in the bunch intensity and in part by decreasing the beam cross section $\left(\eta^{*}\right)$. A new design of the beam optics might require moving the quadrupole triplet closer to the interaction point or installing a D1,3Q,D2 configuration with the last dipole very close to the interaction point. Either may imply a major re-design of the collimators/shielding, and would affect the detector layout at the large $\eta$ limit. For ATLAS, this would add reasons for reducing the angular acceptance, and for modifying the design of the forward quadrupoles. For CMS, this would probably force integration of the forward calorimeter within the forward muon region and might force a further reduction in the acceptance.

Activation of shielding and supports might limit the access-time to the detectors, placing constraints on installation and maintenance scenarios.

\subsubsection{Conclusion on the muon systems}

The modifications needed for the SLHC to the muon systems of ATLAS and CMS should be determined by a cost-benefit analysis depending on the perceived physics potential in the light of results from LHC at $10^{34} \mathrm{~cm}^{-2} \mathrm{~s}^{-1}$. Benchmarking of background simulations from actual LHC experience is also important in deciding how to proceed. In general the choice to be made is between maintaining high- $\eta$ acceptance using new, super-robust, low maintenance detectors, or accepting a reduced high- $\eta$ acceptance limit due to additional shielding that permits the existing LHC muon detector technologies to survive in most locations. The forced re-design of other sub-systems may in any case, ultimately determine the effective high- $\eta$ region accessible for muon detection.

Depending on the exact configuration (particularly high- $\eta$ cut-off) changes in technology might be needed in certain specific regions (eg first forward stations), but the aim should be to use technologies already developed and applied for high-rate tracking at LHC.

To cope with the $80 \mathrm{Mhz}$ bunch-crossing rate, much of the on-board trigger and readout electronics and cooling will have to be replaced, even without change in detector technology. This task may be a major contribution to the re-fit time for SLHC. 


\subsection{Trigger and Data Acquisition}

The consequences are considered for trigger and data-acquisition (TDAQ) systems if LHC is upgraded to have higher luminosity (in the following we assume $10^{35} \mathrm{~cm}^{-2} \mathrm{~s}^{-1}$ ) and a reduced bunch-crossing (BC) period to $12.5 \mathrm{~ns}$. After discussing some issues related to the detectors that also affect TDAQ, we outline the programme of R\&D on TDAQ systems that would be needed for the successful exploitation of such a machine. Finally, we give an indication of the thresholds that might be possible with a first-level (level-1) trigger at SLHC.

\subsubsection{Higher luminosity}

The obvious consequences of raising the luminosity of LHC are higher detector occupancy, increased trigger rates at fixed transverse-momentum thresholds (or higher thresholds for fixed rates), and larger levels of radiation that could damage or perturb the detectors and the on-detector electronics.

\section{a) Occupancy}

Increased occupancy has two important consequences for the TDAQ system: degraded performance of trigger algorithms due to the increase in pile-up, and a larger event size to be read out. Examples of the degradation of the trigger performance include reduced rejection at fixed efficiency from isolation requirements on electron/photon candidates, and increased muon-trigger background rates arising from accidental coincidences between radiation-induced "noise" hits in the muon detectors.

\section{b) Trigger rates}

The increased event size reduces the maximum allowed level-1 rate for fixed readout bandwidth. This suggests that one should perhaps try at least to avoid increasing level-1 rate beyond the maximum of $100 \mathrm{kHz}$ presently envisaged in ATLAS and CMS. Such a strategy appears to be possible, as discussed later, but implies raising the transverse-momentum thresholds on candidate electrons, photons, muons, etc., and using less inclusive triggers. The increase in the thresholds has to compensate for the larger interaction rate, and also for the degradation in algorithm performance due to the higher occupancy (less rejection for fixed efficiency).

\section{c) Radiation damage}

The increased levels of radiation at SLHC could cause problems in terms of damage to detectors and to the on-detector electronics (either permanent damage or single-event-upset effects). Note that part of the level-1 trigger electronics in both ATLAS and CMS is mounted on the detectors. The radiation tolerance of this electronics, as well as the front-end electronics of the detector systems, would need to be assessed in view of increased radiation levels at SLHC. However, it should be kept in mind that the actual radiation levels are presently uncertain ("safety factors" are applied in qualifying the electronics), and will only be known with precision after LHC starts operation.

\subsubsection{Reduced BC period (12.5 ns)}

A reduction of the $\mathrm{BC}$ period below its present value of $25 \mathrm{~ns}$ has important consequences for the level1 trigger and the detector front-end electronics. The present trigger systems are pipelined processors driven by the 25-ns period (40 MHz) LHC machine clock; they select events indicating exactly which $\mathrm{BC}$ produced the interaction of interest. In the following we assume a $\mathrm{BC}$ period of $12.5 \mathrm{~ns}(80 \mathrm{MHz}$ frequency). Frequencies higher than this would amount to almost continuous beam given the rise times of signals from the detectors and the timing resolution achievable in the detector and trigger electronics. The present strategies for timing-in and time monitoring of the experiments rely on using the bunch structure of the machine. This may simply not be possible for BC intervals of less than about $12.5 \mathrm{~ns}$.

From the point of view of performance, it would be advantageous to rebuild the level-1 processor systems to work with data sampled at $80 \mathrm{MHz}$ (internally some data movement and/or processing are already done at $80 \mathrm{MHz}$ and above in both the ATLAS and CMS systems). This would optimise the 
performance of the algorithms (rate versus efficiency) by limiting the effects of pile-up that become more important at SLHC. This provides the best chance to hold the level-1 output rate below $100 \mathrm{kHz}$, leading to cost savings elsewhere by avoiding replacement of the front-end and readout electronics, and avoiding an increase in bandwidth and processing power in the high-level trigger and DAQ systems. It is the only way to identify with $12.5 \mathrm{~ns}$ precision the $\mathrm{BC}$ that caused the trigger.

In some cases the so-called "trigger primitive" information from the detectors (e.g. energy in calorimeter trigger towers) could still be derived from existing detector front-end systems. For example, the trigger primitive generation electronics that prepares the trigger tower data from the CMS digital calorimeter front-end electronics could possibly be modified to calculate the energy deposited in each $12.5 \mathrm{~ns} \mathrm{BC}$ period from the time sequence of measurements made at $40 \mathrm{MHz}$ frequency.

An alternative would be to keep some of the level-1 trigger processor electronics clocked at $25 \mathrm{~ns}$. Here the trigger-primitive information from the detectors (e.g. energy in calorimeter trigger towers) from pairs of BCs would be assigned to $25 \mathrm{~ns}$ intervals. This would still require modifications to the front-end part of the level-1 trigger, but part of the processing chain could be retained as is. The trigger would then identify pairs of BCs to be read out (and where appropriate with data from surrounding BCs) forming so-called time-frames. The data from these time-frames could then be used to reconstruct the hit time in the higher-level triggers and offline - note that for many detectors the resolution is better than $12.5 \mathrm{~ns}$. Drawbacks of this approach are increased pile-up (since the activity from pairs of BCs is combined at least at the level of the trigger processing), and a larger event size (since the size of time-frame has to be enlarged to allow for the ambiguity in the $\mathrm{BC}$ that caused the trigger).

\subsubsection{Comments on detectors (for 12.5 ns BC interval)}

The inner-tracking detectors will have to be replaced for operation at SLHC along with their front-end electronics (radiation damage, occupancy). The new detectors and associated electronics with $12.5 \mathrm{~ns}$ sampling period can benefit from a level-1 trigger that identifies the $\mathrm{BC}$ with $12.5 \mathrm{~ns}$ precision.

Although it may be possible to retain the existing calorimeters, it may be necessary to re-optimize the shaping time of the analogue electronics. It might be possible to retain the existing front-end and readout electronics, provided the analysis can be done with data sampled at $25 \mathrm{~ns}$ period. The time of deposition of the energy in the calorimeter can be reconstructed with high precision using data from a series of measurements in time. With the digital calorimeter readout in CMS, the digital processing that prepares the level-1 trigger tower data would have to be modified to calculate the energy in each $12.5 \mathrm{~ns}$ BC period. ATLAS has a separate system of ADCs for the trigger. Of course, the survival and operability of the on-detector electronics in the higher radiation environment would need to be checked.

For the muon spectrometers, it may be possible to retain (some of) the detectors and associated front-end and readout electronics. In some cases (e.g. ATLAS TGCs), the time resolution of the detectors may be marginal to trigger unambiguously on bunch crossings separated by $12.5 \mathrm{~ns}$. As for the calorimeters, the survival and operability of the on-detector electronics in the higher radiation environment would need to be checked. The rate of spurious triggers induced by radiation in the cavern would also need to be checked.

\subsubsection{Trigger menu}

Three types of triggers will most likely be needed at a SLHC:

- Triggers for very high- $p_{T}$ discovery physics. These do not cause big rate problems since thresholds can be as high as several hundreds of $\mathrm{GeV}$.

- Triggers to complete the LHC physics programme, e.g. precise measurements of the Higgs sector. These require thresholds on leptons/photons/jets as low as those used at the LHC. However, since the final states to be studied are known, one can use exclusive menus (e.g. one lepton plus two b-jets plus missing energy) targeted to the final states that need to be studied. 
- Control/calibration triggers with low thresholds, selecting, e.g., W, Z and top events. These can be pre-scaled.

A first, very preliminary, study has been made to determine the expected rate of some basic inclusive triggers at level-1. An illustrative set of selection criteria is as follows:

- inclusive single muon $p_{T}>30 \mathrm{GeV}$ (rate $\sim 25 \mathrm{kHz}$ );

- inclusive isolated $e / \gamma E_{T}>55 \mathrm{GeV}$ (rate $\sim 20 \mathrm{kHz}$ );

- isolated $e / \gamma$ pair $E_{T}>30 \mathrm{GeV}($ rate $\sim 5 \mathrm{kHz})$ f $^{4}$

- muon pair $p_{T}>20 \mathrm{GeV}$ (rate $\sim$ few kHz?);

- jet $E_{T}>150 \mathrm{GeV}$.AND. $E_{T}^{\text {miss }}>80 \mathrm{GeV}$ required in coincidence (rate $\sim 1-2 \mathrm{kHz}$ );

- inclusive jet trigger $E_{T}>350 \mathrm{GeV}$ (rate $\sim 1 \mathrm{kHz}$ );

- inclusive $E_{T}^{\text {miss }}>150 \mathrm{GeV}$ (rate $\lesssim 1 \mathrm{kHz}$ );

- a multi-jet trigger with thresholds determined by the affordable rate (still to be evaluated).

The rates are very preliminary estimates based on scaling rates from the ATLAS and CMS level-1 trigger TDRs, not allowing for the degradation in performance of isolation, etc. due to the higher level of pile-up at SLHC. This is particularly true for the muon rates, which do not take into account the degradation in performance of the trigger with $p_{T}$ (i.e. the threshold is less sharp at higher $p_{T}$ ), or the possibility of large rates from the increased background due to radiation in the cavern (which could be a serious problem for the inclusive muon trigger in ATLAS). We guess there might be some chance to lower the dimuon threshold, but we err on the safe side for now.

There will certainly be triggers in addition to the above, for example a trigger requiring a muonelectron pair with a $p_{T}$ threshold of about $20-30 \mathrm{GeV}$ for each lepton is likely to have an affordable rate.

\subsubsection{Data Acquisition}

In spite of the continuous and extraordinary evolution of the computing and communication technologies, a research and development programme is necessary in the following domains:

a) Readout network: implementation has to follow the LHC machine luminosity thus exploiting the parallel evolution of technologies The main building block of any LHC data acquisition system is the network interconnecting the data sources (detector digitizers) to the processing nodes (event filters). While processor farms are becoming off-the-shelf commercial components, the same is not yet true for the interconnection technologies whose progress, even if impressive, started more recently than the one in the field of computing. For example today a full commercial network system with the performance required to build a LHC data acquisition network is not yet available in the market (i.e. a switch with thousand ports, non-blocking, 1 Terabit/s aggregate bandwidth etc.). Therefore implementations of the event builders at LHC will be made via subsequent upgrades following both the machine luminosity and (we hope in phase) the evolution of the communication technologies.

The network technologies should be tracked. The new implementations should be applied in the running data acquisition readout systems. In particular the integration of the $10 \mathrm{~Gb} / \mathrm{s}$ Ethernet and the emerging Infiniband technologies should be tested to interconnect large farms of processors (e.g. the farms foreseen for the LHC computing and the Grid projects can provide suitable test beds).

b) Complexity handling, critical at the start of the experiment, because the management of such a large system is a real new problem ('opening a new airport syndrome' e.g. Malpensa) The online computing systems will most likely have more than $10000 \mathrm{CPUs}$. In addition to the hardware boxes (CPUs) there will be millions of software boxes (jobs) to be managed and controlled. These numbers are sufficiently large that the designers will have to confront reliability problems not seen in any previous

\footnotetext{
${ }^{4}$ The isolated $e / \gamma$ pair trigger with an $E_{T}$ threshold of $30 \mathrm{GeV}$ on each cluster could be replaced by a trigger with two different thresholds, e.g. $40 \mathrm{GeV}$ and $25 \mathrm{GeV}$, which could be more efficient for studies involving channels such as $H->\gamma \gamma$.
} 
laboratory setup or slow control system. Moreover the experiment control and information systems will need to be accessible to multiple users with different profiles, expertise and therefore access privileges. The hardware and software management of such a large complex is similar to that found in the present Internet Service Provider centers (e.g. 6000 CPUs in google.com search engine).

Modern technologies should be studied to control distributed computing and exploit the Web tools, currently used in the e-commerce world, to implement the experiment high level controls and user interfaces. Under the new run control the handling of future experiments will not be very different from that of an e-commerce company (same problems: security, remote access, databases, world wide access, knowledge data bases, on-line orders etc.). The exploitation of the immense developments ongoing in the domain of e-commerce will open new ways to operate large collaborations and large set of distributed processors.

\subsubsection{Main R\&D issues}

The main $\mathrm{R} \& \mathrm{D}$ issues for the level-1 trigger relate to the reduction in the $\mathrm{BC}$ period. Data movement is probably the biggest issue for processing at $80-\mathrm{MHz}$ sampling rate. Interconnection issues (links, backplanes, etc.) already drive the design of the level-1 processors in ATLAS and CMS with 40-MHz BC rate. Triggers for SLHC would need higher bandwidth and/or more use of zero suppression, data compression, etc. Processing at higher frequencies and with higher input/output data rates to the processing elements also needs to be investigated, although technological advances (FPGAs, etc.) will help here.

Synchronisation (using the TTC system, etc.) becomes an issue for short BC periods. Present strategies for timing-in and time monitoring of the experiments that rely on using the bunch structure of the machine may have to be reviewed. Finally, some detectors currently used in the trigger may be too slow for $12.5 \mathrm{~ns}$ timing precision, requiring $\mathrm{R} \& \mathrm{D}$ on alternatives.

Concerning the high-level triggers and DAQ, the main issue is how to handle the larger bandwidth (rate times event size) at SLHC. Bandwidth is an issue both for readout and for event building. Processing power is likely to be less of an issue assuming continued growth in the performance/price ratio.

\subsection{Electronics for SLHC}

Electronics Technology has consistently developed at a rate described by an empirical relationship, known as 'Moores Law'. This relationship predicts that the minimum feature size in silicon microelectronics circuits will decrease by a factor of two every five years. This trend has proved to be true since the early 1970s. The smallest feature size commercially available today is $0.13 \mu$ and Intel has recently demonstrated a $0.03 \mu$ transistor in the research laboratory. The significance of this continuing trend is that the number of usable logic gates in a microelectronics chip increases by a factor of 4 every 5 years with a corresponding increase in speed and decrease in power dissipation. In addition the time taken for research developments to become viable commercial products is ten years. Hence we can predict with certainty that the trend will continue for at least a further ten years, but that new technology developments will be required to maintain the growth in the electronics industry in the period from ten to twenty years.

The Particle Physics experiments presently in construction for the CERN LHC would not have been possible without the extensive use of microelectronics technology. This technology was developed for the worldwide computer and telecommunications industries. The successful application of these technologies to the requirements of Particle Physics experiments was the result of an intensive R\&D programme approved by the CERN DRDC. Without this programme the present generation of experiments would not have been possible.

For SLHC it will be necessary to build on the expertise and infrastructure that has been established

for LHC, both at CERN and at the network of collaborating Institutions throughout the international Particle Physics research community. 


\subsubsection{Proposed $R \& D$ Projects}

One of the major successes of the LHC development programme was the demonstration that commercially available $0.25 \mu$ CMOS technology can be radiation hard [60]. This technology is now used extensively in LHC applications with very significant performance and financial gains.

Many radiation-hard circuits for the LHC experiments have been, or are being, converted into DSM technology. Radiation effects can be divided into two categories: total dose effects and single event effects.

Total dose effects in CMOS are mainly associated with charging-up of oxides. During irradiation electrons and holes are generated in the oxides. While the electrons are evacuated rapidly (within ns) holes accumulate in traps leading to transistor threshold shifts. As the oxides get thinner the charging decreases in proportion to the volume of the oxide. Hence thinner oxides are inherently more radiation hard. As the oxide thickness falls below $10 \mathrm{~nm}$ the reduction in the radiation induced threshold voltage becomes even more pronounced [61]. Therefore for deep sub-micron processes (a $0.25 \mu \mathrm{m}$ process has a gate oxide thickness of $5 \mathrm{~nm}$ ) radiation induced threshold voltage shift becomes negligible even at very high radiation doses. However there is still the possibility of leakage paths from drain to source and from one transistor to another that have to be eliminated by special layout techniques [62].

Single event effects will perhaps be the ones that cause the most difficulty for the tracker electronics at SLHC. The effects comprise:

- single event gate rupture that only manifests itself above a critical threshold electric field and should not be an issue for deep sub-micron CMOS circuits.

- single event latch-up that can probably be avoided by the use guard rings that are also used to limit total dose effects.

- single event upsets that cause the logical level of the node to switch state. This effect occurs above a threshold LET. The threshold LET tends to decrease for smaller feature sizes and is a real concern for deep sub-micron circuits.

The more recently available technologies $(0.13 \mu$ and beyond) will require characterisation for SLHC applications and the development of the new design techniques and the required libraries. Understanding the limits, and applicability, of DSM electronics should be the subject of vigorous R\&D for SLHC and for the upgrades of LHC experiments.

Data rates in SHLC detectors will scale with luminosity. This raises the issue of whether to process data at the detector and reduce data volumes before transfer to Off-Detector electronics, or whether to invest in advanced data link technology to minimise the risk to electronics on the detector.

The development of intelligent architectures to reduce the volume of data transferred off the detector; especially in the case of high granularity detectors (Tracker and Pixel Systems) will require common development projects.

The development of very high-speed data links for Particle Physics applications, based on the commercial developments is a common development project. Commercial developments are not optimised for extreme environments found in LHC experiments.

Another consequence of the very high data rates anticipated at Super LHC is the power dissipated in the CMOS electronics in the detectors. There are alternative technologies that could be considered (for example $\mathrm{Si}-\mathrm{Ge}$ ), but the LHC research community has little experience of designing in these technologies. In addition, the advantages of working with a modern commercial process may be lost.

The understanding of the issues involved in using alternative technologies will require substantial work. In addition, more work on power removal techniques in the environment found at LHC will be required.

In the development of electronics for LHC the most pressing problems were those of developing electronics that would operate inside the LHC detectors. As a result of the DRDC programme, these 
problems were solved, but in many cases the overall systems design issues did not receive the required attention.

A new R\&D initiative should recognise this shortcoming and encourage the research community to focus on the systems design issues from the outset. The ultimate performance of a detector system is often limited by the noise that is generated by non-optimal grounding systems. Understanding all the systems issues is the focus of another $\mathrm{R} \& \mathrm{D}$ project that will develop common solutions where possible.

\subsubsection{Organisational Issues}

When the initial DRDC projects were approved in 1989, most of the microelectronics were produced on either 4 or 6 inch wafers. In addition most of the Particle Physics community could obtain access to the best available design software tools through the Europractice programme which made these tools available to the European Teaching and Research community at very low cost.

The $0.25 \mu$ CMOS technology used in many LHC experiments is now produced on 8 inch wafers and the next technology to be used $(0.13 \mu \mathrm{CMOS})$ will soon be produced on 12 inch wafers. In addition the number of interconnection planes is also increasing to give the designer more freedom in connecting the elements within the circuit.

The result of these developments is much more efficient chip designs and much cheaper chips for the very large users (Computer and Telecommunication Industries) who have been driving the development of the technology. Not only will the wafers be more expensive, but the number of masks used in the processing will also increase, which in turn will increase the Non-Recurrent Engineering (NRE) costs. The potential complexity of the designs will also increase together with the complexity of the techniques required to layout and simulate the behaviour of the circuits. The commercial cost of the design software will be very high ( $\gg 1 \mathrm{M}$ dollars), and hence it is crucial to maintain access to the Europractice programme.

The number of research organisations, world-wide, that will be able to access these technologies will be very small and will probably be led by the major Particle Physics Laboratories, where CERN has a leading role. Without the establishment of a world-wide network, involving both the Particle Physics research community and commercial partners, to develop the next generation of electronics, future Particle Physics experiments will not be possible.

Experience with the development required for the LHC implies that the time required to develop the electronics for Super LHC will be $\sim 8-9$ years.

In the mid 1980s the CERN structure was changed to recognise the importance of co-locating a critical mass of the best electronics engineers to develop the electronics required for LHC. The developments required for SLHC will also require an equivalent change in structure at CERN to provide not only critical mass of engineers and the required infrastructure, but also the focus for the required world-wide network within the research community.

If successful, not only will CERN provide the leadership of the international Particle Physics community, but it will become the international focus for the development of multi-disciplinary advanced instrumentation.

\subsection{Conclusions: Experimental Challenges and the Detector R\&D}

A luminosity upgrade of the LHC to $10^{35} \mathrm{~cm}^{-2} \mathrm{~s}^{-1}$ will require significant detectors R\&D especially for the inner tracking systems including that for radiation-hard front-end electronics and optical links. CERN should launch a new R\&D programme as soon as resources allow. This should be modeled on the Detector R\&D Committee programme of the 1990's, but initially with most of the R\&D targeted to the needs for the SLHC.

In the immediate future the highest priority should be given to the completion of the current de- 
tectors and only a very limited R\&D effort should be considered at CERN. However there are several reasons to continue with minimal effort; outside CERN numerous groups are performing generic R\&D. The (S)LHC community will benefit from having a good contact with these groups; the SLHC challenges can provide guidelines for the R\&D effort; CERN can provide test beam and irradiation facilities for these groups and can be an important reference for these groups when they define their national projects; finally this type of work attracts instrumentation students in general and also experts outside the traditional HEP community.

A low-level of human and financial resources should be made available from CERN mainly for co-ordination of several R\&D programs financed by member states on a national basis. A significant increase of activity and therefore resources should be planned for the years 2006 and beyond to give an appropriate impetus for focused activities in view of an SLHC running in the early part of the next decade. If the new R\&D programme is successful, not only will CERN provide the leadership of the international Particle Physics community, but it will become the international focus for the development of multi-disciplinary advanced instrumentation.

Below we draw the conclusions for each of sub-detectors considered above.

\subsubsection{Inner Tracking}

The current ATLAS and CMS trackers have to be completely rebuilt for SLHC in order to withstand a factor 10 higher luminosities. The general approach suggested is to:

a) further develop with industry the current silicon strip technology for use at radii $>60 \mathrm{~cm}$.

b) further develop the current pixel technology that is expected to work at radii between $20 \mathrm{~cm}$ and $60 \mathrm{~cm}$.

c) for the vertex region $(\mathrm{R}<20)$ new concepts and new materials are required to attain the necessary speed and radiation hardness.

Furthermore, there is a need for engineering studies related to materials, power distribution, cooling and development of radiation hard electronics together with a full readout scheme

\subsubsection{Calorimetry}

The calorimetry in the barrel regions of ATLAS and CMS should be able to withstand the ten times higher luminosities. However careful attention has to be paid to the endcap and forward regions.

For liquid argon calorimetry the issues to be investigated are space charge effects and current induced voltage drops amongst others. Operation using different liquids, such as krypton, or even dense cold gases should be evaluated.

For the CMS lead tungstate crystal calorimeter a programme of irradiations emulating SLHC conditions has to be carried to evaluate the performance of the crystals, photodetectors and the front-end electronics.

For the CMS endcap HCAL, short of using a novel technique, methods should be developed for mitigating the effects of higher radiation levels. These could include a combination of a) individual readout of scintillator layers, b) periodic replacement of scintillators, and c) development of a more radiation-tolerant scintillator. For the CMS forward calorimeter replacement of the plastic-clad quartz fibres by quartz-clad quartz fibres has to be envisaged. Development of new techniques should also be pursued.

\subsubsection{Muon Systems}

The LHC experiment muon systems have been designed according to conservative assumptions in the background rates (factor 3 to 5 safety margin above estimates from simulations). The real safety margin 
can only be established from measurements once LHC operates. The simplest viable modification for SLHC is to increase shielding around the beam-pipe at high- $\eta$. The penalty is a cut in the high- $\eta$ acceptance. The modifications to the ATLAS \& CMS muon systems needed for SLHC should be determined by a cost-benefit analysis depending on the perceived physics potential in the light of results from LHC at $10^{34} \mathrm{~cm}^{-2} \mathrm{~s}^{-1}$. In general the choice to be made is between maintaining high- $\eta$ acceptance using new, robust, low maintenance detectors, or accepting a reduced high- $\eta$ acceptance limit due to additional shielding that permits the existing LHC muon detector technologies to survive in most locations. In the worst case the first forward muon stations might need to be replaced by a different design concept. However, technologies can be used that are already proven at LHC in a worse environment, eg those that were candidates for central tracking.

\subsubsection{Trigger and Data Acquistion}

A reduction of the $\mathrm{BC}$ period below its present value of $25 \mathrm{~ns}$ has important consequences for the level1 trigger and the detector front-end electronics. From the point of view of performance, it would be advantageous to rebuild the level-1 processor systems to work with data sampled at $80 \mathrm{MHz}$. Concerning the high-level triggers and DAQ, the main issue is how to handle the larger bandwidth (rate and event size) at SLHC. Bandwidth is an issue both for readout and for event building. In spite of the continuous and extraordinary evolution of the computing and communication technologies, a research and development programme is necessary in the domains of readout network and complexity handling. The network technologies should be tracked. Modern technologies should be studied to control distributed computing and exploit the Web tools.

\subsubsection{Electronics}

The successful application of microelectronics technologies to the requirements of Particle Physics experiments was the result of an intensive R\&D programme approved by the CERN DRDC. One of the major successes of the LHC development programme was the demonstration that commercially available $0.25 \mu$ CMOS (DSM) technology can be radiation hard. This technology is now used extensively in LHC applications with very significant performance and financial gains. The more recently available technologies ( $0.13 \mu$ and beyond) will require characterisation for SLHC applications and the development of the new design techniques and the required libraries. The $0.13 \mu \mathrm{m}$ CMOS will be produced on 12 inch wafers. In addition the number of interconnection planes is also increasing to give the designer more freedom in connecting the elements within the circuit. The Non-Recurrent Engineering (NRE) and the costs of the design software will be very high, and hence it is crucial to maintain access to the Europractice programme. Understanding the limits, and applicability, of DSM electronics should be the subject of vigorous R\&D for SLHC and for the upgrades of LHC experiments. Another vital subject for R\&D is the development of very high-speed data links for Particle Physics applications, based on the commercial developments.

All of the new R\&D initiatives should encourage the research community to focus on the systems design issues from the outset.

\section{CONCLUSIONS}

The physics potential of an upgraded LHC running at a luminosity of $10^{35} \mathrm{~cm}^{-2} \mathrm{~s}^{-1}$ can be summarised as follows:

- The measurement of some of the TGC's will reach an accuracy comparable with the size of EW, and possibly SUSY, virtual corrections.

- New rare decay modes of the SM Higgs boson will become accessible, e.g. $H \rightarrow \mu^{+} \mu^{-}$and $H \rightarrow Z \gamma$. The determination of the Higgs couplings to bottom and top quarks, as well as to EW gauge bosons, will reach precisions of $10 \%$ or better, over a good fraction of the $m_{H}<200 \mathrm{GeV}$ 
mass range. In the MSSM, the region of SUSY parameter space where at least two Higgs bosons will be observed is significantly enlarged relative to the LHC reach.

- The first observation of SM Higgs pair production may be possible in the $170<m_{H}<200 \mathrm{GeV}$ mass range, with a determination of the Higgs self-coupling $\lambda_{H H H}$ at a level of $19 \%$ (25\%) for $m_{H}=170 \mathrm{GeV}\left(m_{H}=200 \mathrm{GeV}\right)$, after background subtraction. The precise size of the backgrounds has however large theoretical uncertainties. The use of data control samples will be necessary to fully pin down these uncertainties and to strenghten the estimates of the significance of the $H H$ signal.

- In the absence of a Higgs signal, studies of resonant and non-resonant scattering of electroweak vector boson pairs at high mass will benefit from the larger statistics, which should give access to a larger variety of channels and in general to more convincing signals than at the LHC. These conclusions, however, depend upon the possibility of maintaining adequate forward jet tagging performances.

- The FCNC decay modes of the top quark $t \rightarrow \gamma / Z q$ may be accessible if their BR is of order $10^{-6}$. This range is of relevance for some theories beyond the Standard Model.

- The mass reach for squarks and gluinos will be extended from $\sim 2.5 \mathrm{TeV}$ (standard LHC) to $\sim 3 \mathrm{TeV}$ (SLHC). In addition, some exclusive SUSY channels which are rate-limited at the standard LHC could be studied in detail with a tenfold increase in statistics, thereby providing additional information about the underlying theory.

- The mass reach for new gauge bosons, or for signatures of Extra-dimension models, will be extended by approximately $30 \%$ relative to the LHC; in the case of compositeness, the sensitivity to deviations from the expected behaviour of quarks in the SM will be extended from a scale $\Lambda=40 \mathrm{TeV}$ to $\Lambda=60 \mathrm{TeV}$.

All of the above can be obtained at a moderate extra cost relative to the overall initial LHC investment, extending the lifetime of the LHC complex, completing its physics potential, and bridging the time gap with future activities.

With the exception of final states containing very energetic objects (e.g. jets, photons or muons with transverse energies in the $\mathrm{TeV}$ range), the feasibility of the above physics programme requires detector upgrades able to maintain the performances expected at the standard $10^{34} \mathrm{~cm}^{-2} \mathrm{~s}^{-1}$ luminosity. In many of the examples discussed in this document, the performance of the LHC detectors is affected not only by the high-luminosity environment, but also by the intrinsic detector limitations in terms of detection efficiency and measurement accuracies. Future studies should therefore aim at identifying an optimal technological and financial balance between luminosity upgrade and detector upgrades, with the goal of maximising the overall physics performance.

The foreseen detector upgrades will require significant detector $R \& D$, especially for the inner tracking systems (including radiation-hard front-end electronics and optical links). CERN should launch a new R\&D programme as soon as resources allow. This should be modeled on the Detector R\&D Committee programme of the 1990's. We believe that a vigorous R\&D activity for the SLHC will entail general and significant progresses in the area of particle detector developments, and therefore will ultimately have impacts on future machines (e.g. a VLHC) and on particle physics in general.

\section{Acknowledgements}

We thank M. Battaglia, C. Da Via, E. Heijne, R. Horisberger, P. Jarron, B. McElrath, M. Moll, T. Rizzo, P. Weilhammer and R. Wunstorf for their contributions to this document.

\section{References}

[1] ATLAS Collaboration, "Detector and physics performance Technical Design Report", CERN/LHCC/99-15. 
[2] CMS Collaboration, Technical Proposal, CERN/LHCC 94-38.

[3] A. De Roeck, J. R. Ellis and F. Gianotti, hep-ex/0112004.

[4] G. Azuelos et al., "Physics in ATLAS at a possible upgraded LHC", ATLAS Internal Note ATLPHYS-2001-002.

[5] U. Baur et al., hep-ph/0201227.

[6] O. Brüning et al., "LHC Luminosity and Energy Upgrade: A Feasibility Study", LHC Project Report in preparation.

[7] E. Richter-Was, D. Froidevaux, L. Poggioli, 'ATLFAST 2.0: a fast simulation package for ATLAS', ATLAS Internal Note ATL-PHYS-98-131 (1998).

[8] D. Abbaneo et al., ALEPH, DELPHI, L3, OPAL Collaborations and LEP Electroweak Working Group and SLD Heavy Flavor and Electroweak Groups, CERN-EP-2001-098 [hep-ex/0112021].

[9] S. Haywood et al., hep-ph/0003275, in "Standard model physics (and more) at the LHC" G. Altarelli and M. L. Mangano eds., Geneva, Switzerland: CERN (2000) 529 p.

[10] R. Heuer et al., TESLA Technical Design Report, hep-ph/0106351.

[11] L. J. Dixon, Z. Kunszt and A. Signer, Phys. Rev. D 60 (1999) 114037. J. Ohnemus and J. F. Owens, Phys. Rev. D 43 (1991) 3626. B. Mele, P. Nason and G. Ridolfi, Nucl. Phys. B 357 (1991) 409. J. Ohnemus, Phys. Rev. D 44 (1991) 3477. S. Frixione, P. Nason and G. Ridolfi, Nucl. Phys. B 383 (1992) 3. J. Ohnemus, Phys. Rev. D 44 (1991) 1403. S. Frixione, Nucl. Phys. B 410 (1993) 280. J. M. Campbell and R. K. Ellis, Phys. Rev. D 60 (1999) 113006.

[12] A. S. Belyaev et al., Phys. Rev. D 59 (1999) 015022.

[13] J. Bagger, S. Dawson, and G. Valencia, Nucl. Phys. B399, 364 (1993).

[14] J. Bagger, et al. Phys. Rev. D49, 1246 (1994); idem Phys. Rev. D52, 3878 (1995).

[15] A. Dobado, D. Espriu, and M. J. Herrero, Z. Phys. C50, 205 (1991); A. Dobado and M. T. Urdiales, Z. Phys. C17, 965 (1996); A. Dobado, M. J. Herrero, E. Ruiz, M. T. Urdiales, and R. Pelaez, Phys. Lett. B352, 400 (1995).

[16] P. Hernandez and J. Vegas, Phys. Lett. B307, 116 (1993); S. Lietti, O. J. P. Éboli, M. C. GonzalezGarcia, and S. F. Novaes, Phys. Lett. B339, 119 (1994); A. Brunstein, O. J. P. Éboli, and M. C. Gonzalez-Garcia, Phys. Lett. B375, 233 (1996); S. Alam, S. Dawson, and R. Szalapski, Phys. Rev. D57, 1577 (1998).

[17] D. Zeppenfeld, R. Kinnunen, A. Nikitenko and E. Richter-Was, Phys. Rev. D 62 (2000) 013009.

[18] T. Plehn and D. Rainwater, Phys. Lett. B 520 (2001) 108.

[19] T. Han and B. McElrath, hep-ph/0201023.

[20] M. Hohlfeld, "On the determination of Higgs parameters in the ATLAS experiment at the LHC", ATLAS Internal Note ATL-PHYS-2001-004.

[21] For a recent review and update of production cross-sections, see: A. Djouadi, W. Kilian, M. Muhlleitner and P. M. Zerwas, Eur. Phys. J. C 10 (1999) 45.

[22] E. W. Glover and J. J. van der Bij, Nucl. Phys. B 309 (1988) 282. 
[23] S. Dawson, S. Dittmaier and M. Spira, Phys. Rev. D 58 (1998) 115012.

[24] A. Dobrovolskaya and V. Novikov, Z. Phys. C 52 (1991) 427. D. A. Dicus, K. J. Kallianpur and S. S. Willenbrock, Phys. Lett. B 200 (1988) 187. K. J. Kallianpur, Phys. Lett. B 215 (1988) 392. A. Abbasabadi, W. W. Repko, D. A. Dicus and R. Vega, Phys. Lett. B 213 (1988) 386.

[25] V. D. Barger, T. Han and R. J. Phillips, Phys. Rev. D 38 (1988) 2766.

[26] C. G. Papadopoulos, Comput. Phys. Commun. 137 (2001) 247. A. Kanaki and C. G. Papadopoulos, Comput. Phys. Commun. 132 (2000) 306.

[27] M. L. Mangano, M. Moretti and R. Pittau, hep-ph/0108069. F. Caravaglios, M. L. Mangano, M. Moretti and R. Pittau, Nucl. Phys. B 539 (1999) 215. M. L. Mangano, M. Moretti, F. Piccinini, R. Pittau and A. Polosa, in preparation.

[28] M. S. Chanowitz and M. K. Gaillard, Nucl. Phys. B261 (1985) 379.

[29] A. Dobado, M. J. Hererro, J. R. Pelaez and E. Ruiz Morales, Phys. Rev. D62 (2000) 055011.

[30] A. Pukhov et al., COMPHEP - a package for evaluation of Feynman Diagrams and Integration of Multi-particle Phase Space, INP MSU 98-41/542, hep-ph/9908288.

[31] J. A. Bagger et al., Phys. Rev. D52 (1995) 3878.

[32] M. Beneke et al., hep-ph/0003033, in "Standard model physics (and more) at the LHC" G. Altarelli and M. L. Mangano eds., Geneva, Switzerland: CERN (2000) 529 p.

[33] S. I. Bityukov and N. V. Krasnikov, Mod. Phys. Lett. A 13 (1998) 3235; hep-ph/9908402; Nucl. Instrum. Meth. A 452 (2000) 518.

[34] S. R. Slabospitsky and L. Sonnenschein, hep-ph/0201292.

[35] I. Hinchliffe and F.E. Paige, hep-ph/0201141.

[36] M. Battaglia et al., CERN-TH/2001-150, hep-ph/0106204.

[37] N. Arkani-Hamed, S. Dimopoulos and C. Dvali, Phys. Lett. B429 (1998) 263.

[38] L. Randall and R. Sundrum, hep-th/9905521.

[39] B. Allanach et al., "Searching for narrow graviton resonances with the ATLAS detector at the Large Hadron Collider", ATLAS Internal Note ATL-PHYS-2000-029, hep-ph/0006114.

[40] H. Davoudiasl, J.L. Hewett, T.G. Rizzo, Phys. Rev. D63 (2001) 075004, hep-ph/0006041, and private communication.

[41] K. Dienes, E. Dudas and T. Gerghetta, Nucl. Phys. B537, 47 (1999); A. Pomarol and M. Quirós, Phys. Lett. B438, 255 (1998); M. Masip and A. Pomarol, Phys. Rev. D60, 096005 (1999); I. Antoniadis, K. Benakli and M. Quirós Phys. Lett. B460, 176 (1999).

[42] T.G. Rizzo, Phys.Rev. D61 055005 (2000).

[43] We warmly thank Tom Rizzo for providing us with the FORTRAN code for $Z / \gamma$ excitation production and for the help in implementing it.

[44] Inner Detector TDR, ATLAS TDR4-5, CERN/LHCC 97-16 and 97-17

[45] Tracker TDR, CMS TDR5, CERN/LHCC 98-6 and Add 1 CERN/LHCC 2000-016 
[46] RD48 Status Report, CERN/LHCC 2000-009

[47] S. Watts, Proc. of VERTEX 2001 conference.

[48] S. Parker and C. Kenney, to be published in IEEE TNS.

[49] Z. Li, Proc. of 2001 Vienna Conference on Instrumentation.

[50] RD42 Status Report, CERN/LHCC 2002-010

[51] K. Borer et al. Nucl.Instr. and Meth., A 440 (2000) 5, RD39 Collaboration.

[52] RD39 Collaboration, in preparation

[53] Liquid Argon Calorimeter TDR, ATLAS TDR 2, CERN/LHCC 96-41.

[54] Tile Calorimeter TDR, ATLAS TDR 3, CERN/LHCC 96-42.

[55] The Electromagnetic Calorimeter TDR, CMS TDR 4, CERN/LHCC 97-33.

[56] The Hadron Calorimeter TDR, CMS TDR 2, CERN/LHCC 97-31.

[57] N. Akchurin et al, CMS Note 2000-007

[58] Muon Spectrometer TDR, ATLAS TDR 10, CERN/LHCC 97-22.

[59] Muon TDR, CMS TDR 3, CERN/LHCC 97-32.

[60] A. Rivetti at al., 5-th Workshop for LHC Experiments, CERN 99-09 (1999) 157.

[61] M. Campbell et al., Nucl.Instr. and Meth. A473 (2001) 140

[62] P. Jarron et al., Nucl. Phys. B Proc. Suppl 78 (1999) 625 